\title{
On singularly perturbed small step size difference-differential nonlinear PDEs
}

\author{
Stéphane Malek* \\ Université de Lille 1 \\ UFR de mathématiques \\ 59655 Villeneuve d'Ascq Cedex France \\ Stephane.Malek@math.univ-lille1.fr
}

Tel 0320434240

May, 232013

\begin{abstract}
We study a family of singularly perturbed small step size difference-differential nonlinear equations in the complex domain. We construct formal solutions to these equations with respect to the perturbation parameter $\epsilon$ which are asymptotic expansions with 1-Gevrey order of actual holomorphic solutions on some sectors in $\epsilon$ near the origin in $\mathbb{C}$. However, these formal solutions can be written as sums of formal series with a corresponding decomposition for the actual solutions which may possess a different Gevrey order called $1^{+}$-Gevrey in the literature. This phenomenon of two levels asymptotics has been already observed in the framework of difference equations, see [6]. The proof rests on a new version of the so-called Ramis-Sibuya theorem which involves both $1-$ Gevrey and $1^{+}-$Gevrey orders. Namely, using classical and truncated Borel-Laplace transforms (introduced by G. Immink in [18]), we construct a set of neighboring sectorial holomorphic solutions and functions whose difference have exponentially and super-exponentially small bounds in the perturbation parameter.
\end{abstract}

Key words: asymptotic expansion, Borel-Laplace transform, Cauchy problem, difference equation, integrodifferential equation, nonlinear partial differential equation, singular perturbation. 2000 MSC: 35C10, $35 \mathrm{C} 20$.

\section{Introduction}

We consider a family of singularly perturbed small step size difference-differential nonlinear problem of the form

(1) $\epsilon \partial_{t} \partial_{z}^{S} X_{i}(t, z, \epsilon)+a \partial_{z}^{S} X_{i}(t, z, \epsilon)$

$$
=\sum_{\underline{k}=\left(s, k_{0}, k_{1}, k_{2}\right) \in \mathcal{A}_{1}} \frac{b_{\underline{k}}(z, \epsilon)}{t^{s+1}}\left(\partial_{t}^{k_{0}} \partial_{z}^{k_{1}} X_{i}\right)\left(t+k_{2} \epsilon, z, \epsilon\right)+P\left(z, \epsilon, X_{i}(t, z, \epsilon)\right)
$$

*The author is partially supported by the french ANR-10-JCJC 0105 project and the PHC Polonium 2013 project No. 28217SG. 
for given initial data

$$
\left(\partial_{z}^{j} X_{i}\right)(t, 0, \epsilon)=\varphi_{j, i}(t, \epsilon) \quad, \quad 0 \leq i \leq \nu-1,0 \leq j \leq S-1,
$$

where $\epsilon$ is a complex parameter, $S$ is some positive integer, $a \in \mathbb{C}^{*}$ is some complex number with $\arg (a) \neq 0, \nu$ is some integer larger than 2 and $\mathcal{A}_{1}$ is a finite subset of $\mathbb{N}^{4}$ which satisfies the constraints (115). The coefficients $b_{k}(z, \epsilon), \underline{k} \in \mathcal{A}_{1}$ of the linear part belong to $\mathcal{O}\{z, \epsilon\}$ and $P(z, \epsilon, X) \in \mathcal{O}\{z, \epsilon\}[X]$ where $\mathcal{O}\{z, \epsilon\}$ denotes the space of holomorphic functions in $(z, \epsilon)$ near the origin in $\mathbb{C}^{2}$. The initial data $\varphi_{j, i}(t, \epsilon)$ are assumed to be holomorphic on products of two sectors $(\mathcal{T} \cap\{|t|>h\}) \times \mathcal{E}_{i} \subset \mathbb{C}^{2}$, for some $h>0$ large enough, where $\mathcal{T}$ is a fixed open unbounded sector centered at 0 and $\underline{\mathcal{E}}=\left\{\mathcal{E}_{i}\right\}_{0 \leq i \leq \nu-1}$ is a family of open bounded sectors centered at the origin whose union form a covering of $\mathcal{V} \backslash\{0\}$, where $\mathcal{V}$ is some neighborhood of 0 .

In the paper [24], we have considered singular singularly perturbed nonlinear problems

$$
\epsilon t^{2} \partial_{t} \partial_{z}^{S} u_{i}(t, z, \epsilon)=F\left(t, z, \epsilon, \partial_{t}, \partial_{z}\right) u_{i}(t, z, \epsilon)+P\left(t, z, \epsilon, u_{i}(t, z, \epsilon)\right)
$$

for given initial data

$$
\left(\partial_{z}^{j} u_{i}\right)(t, 0, \epsilon)=\phi_{j, i}(t, \epsilon) \quad, \quad 0 \leq i \leq \nu-1,0 \leq j \leq S-1,
$$

where $F$ is some differential operator with polynomial coefficients and $P$ some polynomial. The initial data $\phi_{j, i}(t, \epsilon)$ were assumed to be holomorphic on products $\left(\mathcal{T} \cap\left\{|t|<h^{\prime}\right\}\right) \times \mathcal{E}_{i}$, for some $h^{\prime}>0$ small enough. Under suitable constraints on the shape of the equation (3) and on the initial data (4), we have shown the existence of a formal series $\hat{u}(\epsilon)=\sum_{k \geq 0} h_{k} \epsilon^{k} / k$ ! with coefficients $h_{k}$ belonging to the Banach space $\mathbb{F}$ of bounded holomorphic functions on $\left(\mathcal{T} \cap\left\{|t|<h^{\prime}\right\}\right) \times D(0, \delta)$ (for some $\delta>0$ ) equipped with the supremum norm, solution of (3), which is the $1-$ Gevrey asymptotic expansion of actual holomorphic solutions $u_{i}$ of (3), (4) on $\mathcal{E}_{i}$ as $\mathbb{F}$-valued functions, for all $0 \leq i \leq \nu-1$ (see Definition 41 )).

We mention also the work [21] of A. Lastra and the author, where a $q$-analog of the problem (3), (4) was investigated. The discretization was performed with respect to the variable $t$ meaning that $\partial_{t}$ was replaced by the $q$-difference operator $(f(q t)-f(t)) /(q t-t)$ for a complex $q \in \mathbb{C}^{*}$ with $|q|>1$ (which formally tends to $\partial_{t}$ as $q$ tends to 1 ).

In this work we address the same question as in our previous papers [21], [24], namely our main goal is the construction of actual holomorphic solutions $X_{i}(t, z, \epsilon)$ to the problem (1), (2) on domains $(\mathcal{T} \cap\{|t|>h\}) \times D(0, \delta) \times \mathcal{E}_{i}$ for some small disc $D(0, \delta)$ and the analysis of their asymptotic expansions as $\epsilon$ tends to 0 . More precisely, we can present our main statements as follows.

Main results $W e$ choose a set of directions $d_{i} \in \mathbb{R}, 0 \leq i \leq \nu-1$, such that $d_{i} \neq \arg (a)$ with the property that

$$
d_{i}+\arg (t)-\arg (\epsilon) \in(-\pi / 2, \pi / 2)
$$

for all $\epsilon \in \mathcal{E}_{i}$ and $t \in \mathcal{T} \cap\{|t|>h\}$. We make the assumption that the initial Cauchy data $\varphi_{j, i}(t, \epsilon)$ (given in (2)) can be written as Laplace transforms

$$
\varphi_{j, i}(t, \epsilon)=\int_{L_{d_{i}}} V_{j, i}(\tau, \epsilon) e^{-\frac{t \tau}{\epsilon}} d \tau
$$

on $(\mathcal{T} \cap\{|t|>h\}) \times \mathcal{E}_{i}$ along halflines $L_{d_{i}}=\mathbb{R}_{+} e^{\sqrt{-1} d_{i}}$ for directions $d_{i} \in(-\pi / 2, \pi / 2)$ and as truncated Laplace transforms

$$
\varphi_{j, i}(t, \epsilon)=\int_{0}^{\Gamma_{i} \log \left(\Omega_{i} t / \epsilon\right)} V_{j, i}(\tau, \epsilon) e^{-\frac{t \tau}{\epsilon}} d \tau
$$


on $(\mathcal{T} \cap\{|t|>h\}) \times \mathcal{E}_{i}$, for well chosen complex numbers $\Omega_{i} \in \mathbb{C}$ and $\Gamma_{i} \in L_{d_{i}}$, for directions $d_{i} \in$ $[-\pi,-\pi / 2) \cup(\pi / 2, \pi]$, where $V_{j, i}(\tau, \epsilon)$ are holomorphic functions satisfying the growth constraints (117) and (118).

Then, in Theorem 1, we construct a family of holomorphic and bounded functions $X_{i}(t, z, \epsilon)$, $0 \leq i \leq \nu-1$ on the products $(\mathcal{T} \cap\{|t|>h\}) \times D(0, \delta) \times \mathcal{E}_{i}$ for some radius $\delta>0$ small enough with the property that

$$
\left(\partial_{z}^{j} X_{i}\right)(t, 0, \epsilon)=\varphi_{j, i}(t, \epsilon), 0 \leq i \leq \nu-1,0 \leq j \leq S-1,
$$

and whose differences $X_{i+1}(t, z, \epsilon)-X_{i}(t, z, \epsilon)$ satisfy the exponential and super-exponential flatness estimates (121) and (122) on $\mathcal{E}_{i+1} \cap \mathcal{E}_{i}$. Moreover, for all integers $i \in\{0, \ldots, \nu-1\}$ with $d_{i} \in(-\pi / 2, \pi / 2)$, we prove that $X_{i}(t, z, \epsilon)$ is an actual solution of the problem (1), (2).

In a second step (described in Theorem 2), we show the existence of a formal series

$$
\hat{X}(\epsilon)=\sum_{k \geq 0} H_{k} \frac{\epsilon^{k}}{k !}
$$

whose coefficients $H_{k}$ belong to the Banach space $\mathbb{E}$ of bounded holomorphic functions on $(\mathcal{T} \cap$ $\{|t|>h\}) \times D(0, \delta)$, which solves the equation (1) and is the $1-$ Gevrey asymptotic expansion of $X_{i}$ on $\mathcal{E}_{i}$ as $\mathbb{E}$-valued functions. However, this formal series $\hat{X}(\epsilon)$ and the corresponding functions $X_{i}$ own a fine structure which involves two levels of asymptotics. Namely, $\hat{X}(\epsilon)$ and $X_{i}(t, z, \epsilon)$ can be written as sums

$$
\hat{X}(\epsilon)=a(\epsilon)+\hat{X}^{1}(\epsilon)+\hat{X}^{2}(\epsilon), \quad X_{i}(t, z, \epsilon)=a(\epsilon)+X_{i}^{1}(\epsilon)+X_{i}^{2}(\epsilon)
$$

where $a(\epsilon)$ is a convergent series near $\epsilon=0$ with coefficients in $\mathbb{E}$ and $\hat{X}^{1}(\epsilon)$ (resp. $\hat{X}^{2}(\epsilon)$ ) is a formal series with coefficients in $\mathbb{E}$ which is the $1-$ Gevrey asymptotic expansion (resp. the $1^{+}-$Gevrey asymptotic expansion) of the $\mathbb{E}$-valued function $X_{i}^{1}(\epsilon)$ (resp. $X_{i}^{2}(\epsilon)$ ) on $\mathcal{E}_{i}$ in the sense of Definition 4, for all $0 \leq i \leq \nu-1$. In particular, the coefficients $H_{k}^{2}, k \geq 2$, of the formal series $\hat{X}^{2}(\epsilon)$ satisfy estimates of the following form: there exist two constants $C, M>0$ such that

$$
\left\|H_{k}^{2}\right\|_{\mathbb{E}} \leq C M^{k}(k / \log k)^{k}
$$

for all $k \geq 2$.

We stress the fact that this kind of phenomenon with two levels of asymptotics has already been observed in the framework of linear difference systems of the form $Y(s+1)=A(s) Y(s)$ for meromorphic matrices $A(s)$ at $\infty$ in [6] (see especially Example 1 therein). These authors denote the estimates of the form (5) what they called $1^{+}$-Gevrey type growth since a sequence which is $s$-Gevrey for any $s>1$ is $1^{+}$-Gevrey but a 1 -Gevrey sequence is not necessary $1^{+}$-Gevrey (recall that $s$-Gevrey order means that estimates of the form $C M^{k}(k / e)^{k / s}$ hold for some constants $C, M>0$ ). More recently $\mathrm{G}$. Immink has extended their study to the nonlinear situation and has also proposed a resummation procedure which constructs actual holomorphic solutions of difference systems from formal series solutions using modified Laplace transforms and acceleration kernels, see [18].

The Cauchy problem (1), (2) we consider in this work comes within the framework of the asymptotic analysis of singularly perturbed difference-differential equations with small advance or delay, which becomes a growing domain of research these last years and has numerous applications to engineering problems and biology, see for instance [13] and references therein. 
In the context of differential equations most of the statements in the literature are dedicated to problems of the form

$$
\epsilon \partial_{t} x(t, \epsilon)=f(t, \epsilon, x(t, \epsilon), x(t \pm \delta, \epsilon))
$$

for some vector valued function $f$, where $\epsilon>0$ is a small parameter and $\delta>0$ may or not depend on $\epsilon$, and concern the study of asymptotic behaviour of their solutions $x(t, \epsilon)$ as $\epsilon$ tends to 0 . For general and abstract convergence results we quote [1] and references therein for a historical overview. For the construction of solutions $x(t, \epsilon)$ having asymptotic expansions of the form

$$
x(t, \epsilon)=\sum_{l=0}^{n-1} x_{l}(t) \epsilon^{i}+R_{n}(t, \epsilon)
$$

with error bounds estimates for the remainder $R_{n}$ for some integers $n \geq 2$, we refer to [16] and [31].

In the framework of partial differential equations, we mention [28] and [32] where formal asymptotic expansion like (6) have been obtained for solutions to reaction-diffusion equations with small delay.

Nevertheless, these problems when working with a complex parameter $\epsilon$ and with solutions in analytic functions spaces are still quite unstudied although some important and interesting results have been obtained for small step size difference equations, see [12], [14], [15] and for singularly perturbed elliptic partial differential equations, see [27].

In the following, we explain our main results and the principal arguments needed in their proofs. In a first part, we construct a holomorphic function $V(\tau, z, \epsilon)$ near the origin with respect to $(\tau, z)$ and on a punctured disc with respect to $\epsilon$ which solves an integro-differential problem whose coefficients are meromorphic functions with respect to $(\tau, \epsilon)$ with a pole at $\epsilon=0$, see (108), (109). The main novelty compared to our previous studies on singular perturbation problems, see [21], [23], [24], [25], is that the coefficients of (108) now have at most polynomial growth with respect to $\tau$ on the half plane $\mathbb{C}_{+}=\{\tau \in \mathbb{C} / \operatorname{Re}(\tau) \geq 0\}$ but exponential growth on the half plane $\mathbb{C}_{-}=\{\tau \in \mathbb{C} / \operatorname{Re}(\tau)<0\}$. For suitable initial data satisfying the conditions (117), (118), we show that $V(\tau, z, \epsilon)$ can be analytically continued to functions $V_{i}(\tau, z, \epsilon)$ defined on products $U_{d_{i}} \times D(0, \delta) \times \mathcal{E}_{i}$ where $U_{d_{i}}, 0 \leq i \leq \nu-1$, are suitable open unbounded sectors with small aperture (see Definition 3 and below). If $U_{d_{i}}$ is contained in $\mathbb{C}_{+}$, then $V_{i}(\tau, z, \epsilon)$ has at most exponential growth rate with respect to $(\tau, \epsilon)$, namely there exist $C, K>0$ such that

$$
\sup _{z \in D(0, \delta)}\left|V_{i}(\tau, z, \epsilon)\right| \leq C \exp (K|\tau| /|\epsilon|)
$$

for all $\tau \in U_{d_{i}}$, all $\epsilon \in \mathcal{E}_{i}$. When $U_{d_{i}}$ belongs to $\mathbb{C}_{-}, V_{i}(\tau, z, \epsilon)$ owns an exponential growth rate with respect to $\epsilon$ but a super-exponential growth rate with respect to $\tau$, more precisely there exist $C, K_{1}, K_{2}>0$ with

$$
\sup _{z \in D(0, \delta)}\left|V_{i}(\tau, z, \epsilon)\right| \leq C \exp \left(K_{1} \frac{|\tau|}{|\epsilon|}+\exp \left(K_{2}|\tau|\right)\right)
$$

for all $\tau \in U_{d_{i}}$, all $\epsilon \in \mathcal{E}_{i}$.

In a second part, we construct actual solutions $X_{i}(t, z, \epsilon)$ of our problem (1), (2) as Laplace transforms

$$
X_{i}(t, z, \epsilon)=\int_{L_{\gamma_{i}}} V_{i}(\tau, z, \epsilon) e^{\frac{-t \tau}{\epsilon}} d \tau
$$


along halflines $L_{\gamma_{i}} \subset U_{d_{i}}$, when $U_{d_{i}} \subset \mathbb{C}_{+}$, which defines a bounded holomorphic function on the product $(\mathcal{T} \cap\{|t|>h\}) \times D(0, \delta) \times \mathcal{E}_{i}$, provided that $h>0$ is large enough. On the other hand, when $U_{d_{i}}$ is located in $\mathbb{C}_{-}$, we construct bounded holomorphic functions $X_{i}(t, z, \epsilon)$ as truncated Laplace transform (as introduced by G. Immink in [18])

$$
X_{i}(t, z, \epsilon)=\int_{0}^{\Gamma_{i} \log \left(\Omega_{i} t / \epsilon\right)} V_{i}(\tau, z, \epsilon) e^{\frac{-t \tau}{\epsilon}} d \tau
$$

for some complex numbers $\Omega_{i} \in \mathbb{C}$ and $\Gamma_{i} \in U_{d_{i}}$, for all $(t, z, \epsilon) \in(\mathcal{T} \cap\{|t|>h\}) \times D(0, \delta) \times \mathcal{E}_{i}$.

We stress the fact that these functions (10) do not solve the equation (1), but they share with (9) the following crucial properties for our scope: the functions (cocyle) $G_{i}(\epsilon)=X_{i+1}(t, z, \epsilon)-$ $X_{i}(t, z, \epsilon)$, for $0 \leq i \leq \nu-1$ (with the convention $X_{\nu}=X_{0}$ ) are exponentially flat as $\epsilon$ tends to 0 on $\mathcal{E}_{i+1} \cap \mathcal{E}_{i}$ as $\mathbb{E}$-valued functions, where $\mathbb{E}$ is the Banach space of bounded holomorphic functions on $(\mathcal{T} \cap\{|t|>h\}) \times D(0, \delta)$ equipped with the supremum norm. Moreover, when $U_{d_{i}} \cap U_{d_{i+1}} \neq \emptyset$ and $U_{d_{i}}, U_{d_{i+1}} \subset \mathbb{C}_{-}$, the function $G_{i}(\epsilon)$ is even super-exponentially flat, meaning that there exist $K, L, M>0$ such that

$$
\left\|G_{i}(\epsilon)\right\|_{\mathbb{E}} \leq K \exp \left(-\frac{M}{|\epsilon|} \log \frac{L}{|\epsilon|}\right)
$$

for all $\epsilon \in \mathcal{E}_{i+1} \cap \mathcal{E}_{i}$. In the proof, we use as in [24] deformations of the integration's paths in $X_{i}$ with the help of the estimates (7) and (8) (Theorem 1).

In the last part, we establish a new version of the Ramis-Sibuya theorem (Theorem (RS) in Section 5.1) with two levels of 1 -Gevrey and of $1^{+}$-Gevrey type estimates. It is worthwhile noting that the classical Ramis-Sibuya criterion has shown to be very useful to investigate the phenomenon of the so-called multi-summability (which involves several Gevrey orders) of solutions to systems of meromorphic linear differential equations, see [26], nonlinear equations, see [30], and nonlinear systems of difference equations, see [5].

By applying this criterion to our given cocycle $G_{i}$, we deduce the main result of this paper (Theorem 2), namely the existence of a formal series solution of (1)

$$
\hat{X}(\epsilon)=\sum_{k \geq 0} H_{k} \frac{\epsilon^{k}}{k !} \in \mathbb{E}[[\epsilon]]
$$

which is the $1-$ Gevrey asymptotic expansion of $X_{i}(t, z, \epsilon)$ on $\mathcal{E}_{i}$ for all $0 \leq i \leq \nu-1$ and such that the couple $\left(\hat{X}, X_{i}\right)$ shares a three terms decomposition

$$
\hat{X}(\epsilon)=a(\epsilon)+\hat{X}^{1}(\epsilon)+\hat{X}^{2}(\epsilon), \quad X_{i}(t, z, \epsilon)=a(\epsilon)+X_{i}^{1}(\epsilon)+X_{i}^{2}(\epsilon)
$$

where $a(\epsilon)$ is a convergent series at $\epsilon=0, \hat{X}^{1}(\epsilon)$ is the 1 -Gevrey asymptotic expansion of $X_{i}(\epsilon)$ on $\mathcal{E}_{i}$ and $\hat{X}^{2}(\epsilon)$ is the $1^{+}$-Gevrey asymptotic expansion of $X_{i}^{2}(\epsilon)$ on $\mathcal{E}_{i}$, for any $0 \leq i \leq \nu-1$.

The paper is organized as follows.

In Section 2, we consider parameter depending nonlinear convolution differential Cauchy problems with singular and exponential growing coefficients. We construct solutions of these equations in parameter depending Banach spaces of holomorphic functions on sectors with exponential and super-exponential growths.

In Section 3, we construct holomorphic solutions $X_{i}$ to our problem (1), (2) on some sectors $\mathcal{E}_{i}$ as Laplace transform of solutions to nonlinear convolution problems studied in the section 2 .

In the section 4 , we show flatness estimates of exponential type for the cocycle $G_{i}=X_{i+1}-X_{i}$ 
where the $X_{i}$ are constructed in Section 3. We complete this cocycle on the full family $\mathcal{E}$ with the help of truncated Laplace transform of solutions to singular problems studied in Section 2. Moreover, we give flatness estimates that can be of super-exponential growth on some intersections $\mathcal{E}_{i+1} \cap \mathcal{E}_{i}$.

In the last section, we first state a new version of the Ramis-Sibuya theorem in the framework of $1-$ Gevrey and $1^{+}$-Gevrey type estimates and we finally prove our main result.

\section{A global Cauchy problem with singular complex parameter}

\subsection{Banach spaces of holomorphic functions on sectors with exponential and super-exponential growths}

We denote $D(0, r)$ the open disc centered at 0 with radius $r>0$ in $\mathbb{C}$. Let $S_{d}$ be an open bounded or unbounded sector with bisecting direction $d \in \mathbb{R}$ and $\mathcal{E}$ be an open sector with finite radius $r_{\mathcal{E}}$, both centered at 0 in $\mathbb{C}$. By convention, these sectors do not contain the origin in $\mathbb{C}$. For any open set $\mathcal{D} \subset \mathbb{C}$, we denote $\mathcal{O}(\mathcal{D})$ the vector space of holomorphic functions on $\mathcal{D}$. In this section 2 , we set $\Omega=\left(S_{d} \cup D(0, r)\right) \times \mathcal{E}$. Let $b>1$ a real number and let $r_{b}(\beta)=\sum_{n=0}^{\beta} 1 /(n+1)^{b}$ for all integers $\beta \geq 0$.

Definition 1 Let $\epsilon \in \mathcal{E}$ and $\sigma>0, \sigma^{\prime} \geq 0$ be real numbers. We denote $S E_{\beta, \epsilon, \sigma, \sigma^{\prime}, \Omega}$ the vector space of all functions $v \in \mathcal{O}\left(S_{d} \cup D(0, r)\right)$ such that

$$
\|v(\tau)\|_{\beta, \epsilon, \sigma, \sigma^{\prime}, \Omega}:=\sup _{\tau \in S_{d} \cup D(0, r)}|v(\tau)|\left(1+\frac{|\tau|^{2}}{|\epsilon|^{2}}\right) \exp \left(-\frac{\sigma}{|\epsilon|} r_{b}(\beta)|\tau|-\exp \left(\sigma^{\prime} r_{b}(\beta)|\tau|\right)\right)
$$

is finite. Let $\delta>0$ be a real number. We define $S G\left(\epsilon, \sigma, \sigma^{\prime}, \delta, \Omega\right)$ to be the vector space of all functions $v(\tau, z)=\sum_{\beta \geq 0} v_{\beta}(\tau) z^{\beta} / \beta$ ! that belong to $\mathcal{O}\left(S_{d} \cup D(0, r)\right)\{z\}$ such that

$$
\|v(\tau, z)\|_{\left(\epsilon, \sigma, \sigma^{\prime}, \delta, \Omega\right)}:=\sum_{\beta \geq 0}\left\|v_{\beta}(\tau)\right\|_{\beta, \epsilon, \sigma, \sigma^{\prime}, \Omega} \frac{\delta^{\beta}}{\beta !}
$$

is finite. One can check that the normed space $\left(S G\left(\epsilon, \sigma, \sigma^{\prime}, \delta, \Omega\right),\|\cdot\|_{\left(\epsilon, \sigma, \sigma^{\prime}, \delta, \Omega\right)}\right)$ is a Banach space.

Remark: These norms are appropriate modifications of the norms introduced by G. Immink in [18] and those of the author introduced in the work [24]. Notice that for $\sigma^{\prime}=0$, the space $S G(\epsilon, \sigma, 0, \delta, \Omega)$ coincides with the space $G(\epsilon, \sigma, \delta, \Omega)$ defined in [24].

In the next proposition, we study the rate of growth of the functions belonging to the latter Banach spaces.

Proposition 1 Let $v(\tau, z) \in S G\left(\epsilon, \sigma, \sigma^{\prime}, \delta, \Omega\right)$. Let $0<\delta_{1}<1$. There exists a constant $C>0$ (depending on $\|v\|_{\left(\epsilon, \sigma, \sigma^{\prime}, \delta, \Omega\right)}$ and $\delta_{1}$ ) such that

$$
|v(\tau, z)| \leq C\left(1+\frac{|\tau|^{2}}{|\epsilon|^{2}}\right)^{-1} \exp \left(\frac{\sigma \zeta(b)}{|\epsilon|}|\tau|+\exp \left(\sigma^{\prime} \zeta(b)|\tau|\right)\right)
$$

for all $\tau \in S_{d} \cup D(0, r)$, all $z \in \mathbb{C}$ such that $\frac{|z|}{\delta}<\delta_{1}$, where $\zeta(b)=\sum_{n=0}^{\infty} 1 /(n+1)^{b}$. 
Proof Let $v(\tau, z)=\sum_{\beta \geq 0} v_{\beta}(\tau) z^{\beta} / \beta$ ! be in $S G\left(\epsilon, \sigma, \sigma^{\prime}, \delta, \Omega\right)$. By definition, there exists a constant $c_{1}>0$ (depending on $\|v\|_{\left(\epsilon, \sigma, \sigma^{\prime}, \delta, \Omega\right)}$ ) such that

$$
\left|v_{\beta}(\tau)\right| \leq c_{1}\left(1+\frac{|\tau|^{2}}{|\epsilon|^{2}}\right)^{-1} \exp \left(\frac{\sigma}{|\epsilon|} r_{b}(\beta)|\tau|+\exp \left(\sigma^{\prime} r_{b}(\beta)|\tau|\right)\right) \beta !\left(\frac{1}{\delta}\right)^{\beta}
$$

for all $\beta \geq 0$, all $\tau \in S_{d} \cup D(0, r)$. Let $0<\delta_{1}<1$. From the definition of $\zeta(b)$, we deduce that

$$
\begin{aligned}
|v(\tau, z)| \leq c_{1}\left(1+\frac{|\tau|^{2}}{|\epsilon|^{2}}\right)^{-1} \sum_{\beta \geq 0} & \exp \left(\frac{\sigma}{|\epsilon|} r_{b}(\beta)|\tau|+\exp \left(\sigma^{\prime} r_{b}(\beta)|\tau|\right)\right)\left(\delta_{1}\right)^{\beta} \\
& \leq c_{1}\left(1+\frac{|\tau|^{2}}{|\epsilon|^{2}}\right)^{-1} \exp \left(\frac{\sigma \zeta(b)}{|\epsilon|}|\tau|+\exp \left(\sigma^{\prime} \zeta(b)|\tau|\right)\right) \frac{1}{1-\delta_{1}}
\end{aligned}
$$

for all $z \in \mathbb{C}$ such that $\frac{|z|}{\delta}<\delta_{1}<1$, all $\tau \in S_{d} \cup D(0, r)$. Finally, from (13), we deduce the estimates (12).

In the next proposition, we study some linear operators of multiplication by polynomials and exponential functions acting on the space $S G\left(\epsilon, \sigma, \sigma^{\prime}, \delta, \Omega\right)$.

Proposition 2 Let $k_{0} \geq 0$ be a non negative integer and let $k_{1}, k_{2} \in \mathbb{N}^{*}$.

1) Let $\sigma^{\prime}>0$. Assume that the condition

$$
k_{1} \geq b k_{0}+\frac{k_{2} b}{\sigma^{\prime}}
$$

holds. Moreover, we assume that the function $\tau \mapsto \exp \left(-k_{2} \tau\right)$ is unbounded on the sector $S_{d}$. Then, for all $\epsilon \in \mathcal{E}$, the operator $v(\tau, z) \mapsto \exp \left(-k_{2} \tau\right) \tau^{k_{0}} \partial_{z}^{-k_{1}} v(\tau, z)$ is a bounded linear operator from $\left(S G\left(\epsilon, \sigma, \sigma^{\prime}, \delta, \Omega\right),\|\cdot\|_{\left(\epsilon, \sigma, \sigma^{\prime}, \delta, \Omega\right)}\right)$ into itself. Moreover, there exists a constant $C_{1}>$ 0 (depending on $k_{0}, k_{1}, k_{2}, \sigma, \sigma^{\prime}, b$ ), which does not depend on $\epsilon \in \mathcal{E}$, such that

$$
\left\|\exp \left(-k_{2} \tau\right) \tau^{k_{0}} \partial_{z}^{-k_{1}} v(\tau, z)\right\|_{\left(\epsilon, \sigma, \sigma^{\prime}, \delta, \Omega\right)} \leq C_{1}|\epsilon|^{k_{0}} \delta^{k_{1}}\|v(\tau, z)\|_{\left(\epsilon, \sigma, \sigma^{\prime}, \delta, \Omega\right)}
$$

for all $v \in S G\left(\epsilon, \sigma, \sigma^{\prime}, \delta, \Omega\right)$, all $\epsilon \in \mathcal{E}$.

2) Let $\sigma^{\prime} \geq 0$. Assume that the condition

$$
k_{1} \geq b k_{0}
$$

holds. Then, for all $\epsilon \in \mathcal{E}$, the operator $v(\tau, z) \mapsto \tau^{k_{0}} \partial_{z}^{-k_{1}} v(\tau, z)$ is a bounded linear operator from $\left(S G\left(\epsilon, \sigma, \sigma^{\prime}, \delta, \Omega\right),\|\cdot\|_{\left(\epsilon, \sigma, \sigma^{\prime}, \delta, \Omega\right)}\right)$ into itself. Moreover, there exists a constant $\check{C}_{1}>0$ (depending on $\left.k_{0}, k_{1}, \sigma, b\right)$, which does not depend on $\epsilon \in \mathcal{E}$, such that

$$
\left\|\tau^{k_{0}} \partial_{z}^{-k_{1}} v(\tau, z)\right\|_{\left(\epsilon, \sigma, \sigma^{\prime}, \delta, \Omega\right)} \leq \check{C}_{1}|\epsilon|^{k_{0}} \delta^{k_{1}}\|v(\tau, z)\|_{\left(\epsilon, \sigma, \sigma^{\prime}, \delta, \Omega\right)}
$$

for all $v \in S G\left(\epsilon, \sigma, \sigma^{\prime}, \delta, \Omega\right)$, all $\epsilon \in \mathcal{E}$.

Proof 1) Let $v(\tau, z) \in S G\left(\epsilon, \sigma, \sigma^{\prime}, \delta, \Omega\right)$. By definition, we have

$$
\left\|\exp \left(-k_{2} \tau\right) \tau^{k_{0}} \partial_{z}^{-k_{1}} v(\tau, z)\right\|_{\left(\epsilon, \sigma, \sigma^{\prime}, \delta, \Omega\right)}=\sum_{\beta \geq k_{1}}\left\|\exp \left(-k_{2} \tau\right) \tau^{k_{0}} v_{\beta-k_{1}}(\tau)\right\|_{\beta, \epsilon, \sigma, \sigma^{\prime}, \Omega} \frac{\delta^{\beta}}{\beta !} .
$$


Lemma 1 There exists a constant $C_{1.1}>0$ (depending on $k_{0}, k_{1}, k_{2}, \sigma, \sigma^{\prime}$ ) such that

$$
\left\|\exp \left(-k_{2} \tau\right) \tau^{k_{0}} v_{\beta-k_{1}}(\tau)\right\|_{\beta, \epsilon, \sigma, \sigma^{\prime}, \Omega} \leq C_{1.1}|\epsilon|^{k_{0}}(1+\beta)^{b k_{0}+\frac{k_{2} b}{\sigma^{\prime}}}\left\|v_{\beta-k_{1}}(\tau)\right\|_{\beta-k_{1}, \epsilon, \sigma, \sigma^{\prime}, \Omega}
$$

for all $\beta \geq k_{1}$.

Proof We can write

$$
\begin{aligned}
\left|\exp \left(-k_{2} \tau\right) \tau^{k_{0}} v_{\beta-k_{1}}(\tau)\right| & =\left|\exp \left(-k_{2} \tau\right) \tau^{k_{0}}\right| \\
\times \exp & \left(\frac{\sigma}{|\epsilon|} r_{b}\left(\beta-k_{1}\right)|\tau|+\exp \left(\sigma^{\prime} r_{b}\left(\beta-k_{1}\right)|\tau|\right)\right)\left(1+\frac{|\tau|^{2}}{|\epsilon|^{2}}\right)^{-1} \\
& \times\left|v_{\beta-k_{1}}(\tau)\right|\left(1+\frac{|\tau|^{2}}{|\epsilon|^{2}}\right) \exp \left(-\frac{\sigma}{|\epsilon|} r_{b}\left(\beta-k_{1}\right)|\tau|-\exp \left(\sigma^{\prime} r_{b}\left(\beta-k_{1}\right)|\tau|\right)\right)
\end{aligned}
$$

for all $\tau \in S_{d} \cup D(0, r)$. From this latter equality, we deduce

$$
\left\|\exp \left(-k_{2} \tau\right) \tau^{k_{0}} v_{\beta-k_{1}}(\tau)\right\|_{\beta, \epsilon, \sigma, \sigma^{\prime}, \Omega} \leq A(\epsilon, \beta)\left\|v_{\beta-k_{1}}(\tau)\right\|_{\beta-k_{1}, \epsilon, \sigma, \sigma^{\prime}, \Omega}
$$

where

$$
\begin{aligned}
A(\epsilon, \beta)=\sup _{\tau \in S_{d} \cup D(0, r)} \exp \left(k_{2}|\tau|\right)|\tau|^{k_{0}} \exp \left(\frac{\sigma}{|\epsilon|} r_{b}\left(\beta-k_{1}\right)|\tau|\right. & \left.+\exp \left(\sigma^{\prime} r_{b}\left(\beta-k_{1}\right)|\tau|\right)\right) \\
& \times \exp \left(-\frac{\sigma}{|\epsilon|} r_{b}(\beta)|\tau|-\exp \left(\sigma^{\prime} r_{b}(\beta)|\tau|\right)\right)
\end{aligned}
$$

Lemma 2 The following inequality holds

$$
\sup _{\tau \in S_{d} \cup D(0, r)}|\tau|^{k_{0}} \exp \left(\frac{\sigma}{|\epsilon|} r_{b}\left(\beta-k_{1}\right)|\tau|-\frac{\sigma}{|\epsilon|} r_{b}(\beta)|\tau|\right) \leq\left(\frac{k_{0} e^{-1}}{\sigma k_{1}}\right)^{k_{0}}|\epsilon|^{k_{0}}(1+\beta)^{b k_{0}}
$$

for all $\beta \geq k_{1}$.

Proof From the fact that

$$
r_{b}(\beta)-r_{b}\left(\beta-k_{1}\right) \geq \frac{k_{1}}{(\beta+1)^{b}}
$$

for all $\beta \geq k_{1}$, we deduce that

$$
|\tau|^{k_{0}} \exp \left(\frac{\sigma}{|\epsilon|} r_{b}\left(\beta-k_{1}\right)|\tau|-\frac{\sigma}{|\epsilon|} r_{b}(\beta)|\tau|\right) \leq|\tau|^{k_{0}} \exp \left(-\frac{\sigma}{|\epsilon|} \frac{k_{1}}{(1+\beta)^{b}}|\tau|\right)
$$

for all $\tau \in S_{d} \cup D(0, r)$. From (24) and the classical equality

$$
\sup _{x \geq 0} x^{m_{1}} \exp \left(-m_{2} x\right)=\left(m_{1} / m_{2}\right)^{m_{1}} e^{-m_{1}}
$$

for any real numbers $m_{1}, m_{2}>0$, we get (22).

Lemma 3 There exists a constant $d>0$ (depending on $k_{1}, k_{2}$ and $\sigma^{\prime}$ ) such that

$$
\exp \left(k_{2}|\tau|+\exp \left(\sigma^{\prime} r_{b}\left(\beta-k_{1}\right)|\tau|\right)-\exp \left(\sigma^{\prime} r_{b}(\beta)|\tau|\right)\right) \leq \exp \left(\frac{k_{2} b}{\sigma^{\prime}} \log (1+\beta)+d\right)
$$

for all $\beta \geq k_{1}$, all $\tau \in S_{d} \cup D(0, r)$. 
Proof With the help of the inequality (23) and from the Taylor formula we know that for all $\tau \in S_{d} \cup D(0, r)$, all $\beta \geq 0$ there exists a constant $c \in\left(\sigma^{\prime} r_{b}\left(\beta-k_{1}\right)|\tau|, \sigma^{\prime} r_{b}(\beta)|\tau|\right)$ such that

$$
\begin{aligned}
\exp \left(\sigma^{\prime} r_{b}\left(\beta-k_{1}\right)|\tau|\right) & -\exp \left(\sigma^{\prime} r_{b}(\beta)|\tau|\right) \\
& =\sigma^{\prime}|\tau|\left(r_{b}\left(\beta-k_{1}\right)-r_{b}(\beta)\right) e^{c} \leq-\sigma^{\prime}|\tau| \frac{k_{1}}{(1+\beta)^{b}} \exp \left(\sigma^{\prime} r_{b}\left(\beta-k_{1}\right)|\tau|\right)
\end{aligned}
$$

The next inequality will the useful. Let $a, b, c>0$ be real numbers. Then,

$$
\sup _{x \geq 0} c x-a x e^{b x} \leq \max \left(c, \frac{c}{b}\left(\log \left(\frac{c}{a b}\right)-1\right)\right) .
$$

Indeed, for all $x \geq 1$, we have $c x-a x e^{b x} \leq \psi(x)=c x-a e^{b x}$. If $\log \left(\frac{c}{a b}\right) / b \geq 1$, the function $\psi$ gets it's maximum value $\frac{c}{b}\left(\log \left(\frac{c}{a b}\right)-1\right)$ on $[1,+\infty)$ at $x_{0}=\log \left(\frac{c}{a b}\right) / b$. If $\log \left(\frac{c}{a b}\right) / b<1, \psi$ gets it's maximum value $c-a e^{b} \leq c$ on $[1,+\infty)$ at $x_{0}=1$. On the other hand, for all $0 \leq x \leq 1$, we have that $c x-a x e^{b x} \leq c$.

Using (28), we deduce that

$$
\begin{aligned}
& \sup _{\tau \in S_{d} \cup D(0, r)} k_{2}|\tau|-\sigma^{\prime}|\tau| \frac{k_{1}}{(1+\beta)^{b}} \exp \left(\sigma^{\prime} r_{b}\left(\beta-k_{1}\right)|\tau|\right) \\
& \leq \max \left(k_{2}, \frac{k_{2}}{\sigma^{\prime} r_{b}\left(\beta-k_{1}\right)}\left(\log \left(\frac{k_{2}}{\left(\sigma^{\prime}\right)^{2} k_{1} r_{b}\left(\beta-k_{1}\right)}(1+\beta)^{b}\right)-1\right)\right)
\end{aligned}
$$

for all $\beta \geq k_{1}$. From this latter inequality, we deduce the existence of a constant $d>0$ (depending on $k_{1}, k_{2}$, and $\sigma^{\prime}$ ) such that

$$
\sup _{\tau \in S_{d} \cup D(0, r)} k_{2}|\tau|-\sigma^{\prime}|\tau| \frac{k_{1}}{(1+\beta)^{b}} \exp \left(\sigma^{\prime} r_{b}\left(\beta-k_{1}\right)|\tau|\right) \leq \frac{k_{2} b}{\sigma^{\prime}} \log (1+\beta)+d
$$

for all $\beta \geq k_{1}$. The inequality (26) follows.

Gathering the estimates (22) and (26) yields a constant $C_{1.1}>0$ (depending on $k_{0}, k_{1}, k_{2}, \sigma, \sigma^{\prime}$ ) such that

$$
A(\epsilon, \beta) \leq C_{1.1}|\epsilon|^{k_{0}}(1+\beta)^{b k_{0}+\frac{k_{2} b}{\sigma^{\prime}}}
$$

for all $\beta \geq k_{1}$. From (20) and (29) the lemma 1 follows.

Now, from (18) and (19), we deduce

$$
\begin{aligned}
& \left\|\exp \left(-k_{2} \tau\right) \tau^{k_{0}} \partial_{z}^{-k_{1}} v(\tau, z)\right\|_{\left(\epsilon, \sigma, \sigma^{\prime}, \delta, \Omega\right)} \\
& \quad \leq \sum_{\beta \geq k_{1}} C_{1.1}|\epsilon|^{k_{0}}(1+\beta)^{b k_{0}+\frac{k_{2} b}{\sigma^{\prime}}} \frac{\left(\beta-k_{1}\right) !}{\beta !}\left\|v_{\beta-k_{1}}(\tau)\right\|_{\beta-k_{1}, \epsilon, \sigma, \sigma^{\prime}, \Omega} \delta^{k_{1}} \frac{\delta^{\beta-k_{1}}}{\left(\beta-k_{1}\right) !} .
\end{aligned}
$$

Now, from the assumption (14), we get a constant $C_{1.2}>0$ (depending on $b, k_{0}, k_{1}, k_{2}, \sigma^{\prime}$ ) such that

$$
(1+\beta)^{b k_{0}+\frac{b k_{2}}{\sigma^{\prime}}} \frac{\left(\beta-k_{1}\right) !}{\beta !} \leq C_{1.2}
$$

for all $\beta \geq k_{1}$. Finally, from (30) together with (31) we get (15).

2) Let $v(\tau, z) \in S G\left(\epsilon, \sigma, \sigma^{\prime}, \delta, \Omega\right)$. By definition, we have

$$
\left\|\tau^{k_{0}} \partial_{z}^{-k_{1}} v(\tau, z)\right\|_{\left(\epsilon, \sigma, \sigma^{\prime}, \delta, \Omega\right)}=\sum_{\beta \geq k_{1}}\left\|\tau^{k_{0}} v_{\beta-k_{1}}(\tau)\right\|_{\beta, \epsilon, \sigma, \sigma^{\prime}, \Omega} \frac{\delta^{\beta}}{\beta !} .
$$


Lemma 4 There exists a constant $\check{C}_{1.1}>0$ (depending on $k_{0}, k_{1}, \sigma$ ) such that

$$
\left\|\tau^{k_{0}} v_{\beta-k_{1}}(\tau)\right\|_{\beta, \epsilon, \sigma, \sigma^{\prime}, \Omega} \leq \check{C}_{1.1}|\epsilon|^{k_{0}}(1+\beta)^{b k_{0}}\left\|v_{\beta-k_{1}}(\tau)\right\|_{\beta-k_{1}, \epsilon, \sigma, \sigma^{\prime}, \Omega}
$$

for all $\beta \geq k_{1}$.

Proof The explanation relies on the beginning of Lemma 1 . If one puts $k_{2}=0$ in the estimates (20) one gets

$$
\left\|\tau^{k_{0}} v_{\beta-k_{1}}(\tau)\right\|_{\beta, \epsilon, \sigma, \sigma^{\prime}, \Omega} \leq \check{A}(\epsilon, \beta)\left\|v_{\beta-k_{1}}(\tau)\right\|_{\beta-k_{1}, \epsilon, \sigma, \sigma^{\prime}, \Omega}
$$

where

$$
\begin{aligned}
& \check{A}(\epsilon, \beta)=\sup _{\tau \in S_{d} \cup D(0, r)}|\tau|^{k_{0}} \exp \left(\frac{\sigma}{|\epsilon|} r_{b}\left(\beta-k_{1}\right)|\tau|+\exp \left(\sigma^{\prime} r_{b}\left(\beta-k_{1}\right)|\tau|\right)\right) \\
& \times \exp \left(-\frac{\sigma}{|\epsilon|} r_{b}(\beta)|\tau|-\exp \left(\sigma^{\prime} r_{b}(\beta)|\tau|\right)\right) \leq \sup _{\tau \in S_{d} \cup D(0, r)}|\tau|^{k_{0}} \exp \left(\frac{\sigma}{|\epsilon|} r_{b}\left(\beta-k_{1}\right)|\tau|\right) \\
& \times \exp \left(-\frac{\sigma}{|\epsilon|} r_{b}(\beta)|\tau|\right) .
\end{aligned}
$$

Therefore, Lemma 4 is a consequence of Lemma 2 together with (34), (35).

Now, from (32) and (33), we get

$$
\begin{aligned}
& \left\|\tau^{k_{0}} \partial_{z}^{-k_{1}} v(\tau, z)\right\|_{\left(\epsilon, \sigma, \sigma^{\prime}, \delta, \Omega\right)} \\
& \quad \leq \sum_{\beta \geq k_{1}} \check{C}_{1.1}|\epsilon|^{k_{0}}(1+\beta)^{b k_{0}} \frac{\left(\beta-k_{1}\right) !}{\beta !}\left\|v_{\beta-k_{1}}(\tau)\right\|_{\beta-k_{1}, \epsilon, \sigma, \sigma^{\prime}, \Omega} \delta^{k_{1}} \frac{\delta^{\beta-k_{1}}}{\left(\beta-k_{1}\right) !} .
\end{aligned}
$$

Now, from the assumption (16), we get a constant $\check{C}_{1.2}>0$ (depending on $b, k_{0}, k_{1}$ ) such that

$$
(1+\beta)^{b k_{0}} \frac{\left(\beta-k_{1}\right) !}{\beta !} \leq \check{C}_{1.2}
$$

for all $\beta \geq k_{1}$. Finally, the result follows from (36) and (37).

Proposition 3 Let $k_{0} \geq 0$ be a non negative integer and let $k_{2} \in \mathbb{N}^{*}$.

1) Let $\sigma>\tilde{\sigma}>0, \sigma^{\prime}>\tilde{\sigma}^{\prime}>0$ be real numbers. We assume that the function $\tau \mapsto \exp \left(-k_{2} \tau\right)$ is unbounded on the sector $S_{d}$. Then, there exists a constant $\tilde{C}_{1}>0$ (depending on $k_{0}, k_{2}, \sigma, \tilde{\sigma}, \sigma^{\prime}, \tilde{\sigma}^{\prime}$ ) such that

$$
\left\|\exp \left(-k_{2} \tau\right) \tau^{k_{0}} v(\tau, z)\right\|_{\left(\epsilon, \sigma, \sigma^{\prime}, \delta, \Omega\right)} \leq \tilde{C}_{1}|\epsilon|^{k_{0}}\|v(\tau, z)\|_{\left(\epsilon, \tilde{\sigma}, \tilde{\sigma}^{\prime}, \delta, \Omega\right)}
$$

for all $v \in S G\left(\epsilon, \tilde{\sigma}, \tilde{\sigma}^{\prime}, \delta, \Omega\right)$, all $\epsilon \in \mathcal{E}$.

2) Let $\sigma>\tilde{\sigma}>0$ be real numbers. Then, there exists a constant $\tilde{C}_{1.1}>0$ (depending on $\left.k_{0}, \sigma, \tilde{\sigma}\right)$ such that

$$
\left\|\tau^{k_{0}} v(\tau, z)\right\|_{(\epsilon, \sigma, 0, \delta, \Omega)} \leq \tilde{C}_{1.1}|\epsilon|^{k_{0}}\|v(\tau, z)\|_{(\epsilon, \tilde{\sigma}, 0, \delta, \Omega)}
$$

for all $v \in S G(\epsilon, \tilde{\sigma}, 0, \delta, \Omega)$, all $\epsilon \in \mathcal{E}$. 
Proof 1) Let $v(\tau, z) \in S G\left(\epsilon, \tilde{\sigma}, \tilde{\sigma}^{\prime}, \delta, \Omega\right)$. By definition, we have

$$
\left\|\exp \left(-k_{2} \tau\right) \tau^{k_{0}} v(\tau, z)\right\|_{\left(\epsilon, \sigma, \sigma^{\prime}, \delta, \Omega\right)}=\sum_{\beta \geq 0}\left\|\exp \left(-k_{2} \tau\right) \tau^{k_{0}} v_{\beta}(\tau)\right\|_{\beta, \epsilon, \sigma, \sigma^{\prime}, \Omega} \frac{\delta^{\beta}}{\beta !} .
$$

Lemma 5 There exists a constant $\tilde{C}_{1}>0$ (depending on $k_{0}, k_{2}, \sigma, \tilde{\sigma}, \sigma^{\prime}, \tilde{\sigma}^{\prime}$ ) such that

$$
\left\|\exp \left(-k_{2} \tau\right) \tau^{k_{0}} v_{\beta}(\tau)\right\|_{\beta, \epsilon, \sigma, \sigma^{\prime}, \Omega} \leq \tilde{C}_{1}|\epsilon|^{k_{0}}\left\|v_{\beta}(\tau)\right\|_{\beta, \epsilon, \tilde{\sigma}, \tilde{\sigma}^{\prime}, \Omega}
$$

for all $\beta \geq 0$.

Proof We can write

$$
\begin{aligned}
\left|\exp \left(-k_{2} \tau\right) \tau^{k_{0}} v_{\beta}(\tau)\right| & =\left|\exp \left(-k_{2} \tau\right) \tau^{k_{0}}\right| \\
\times \exp \left(\frac{\tilde{\sigma}}{|\epsilon|} r_{b}(\beta)|\tau|+\exp \left(\tilde{\sigma}^{\prime} r_{b}(\beta)|\tau|\right)\right)\left(1+\frac{|\tau|^{2}}{|\epsilon|^{2}}\right)^{-1} & \quad \times\left|v_{\beta}(\tau)\right|\left(1+\frac{|\tau|^{2}}{|\epsilon|^{2}}\right) \exp \left(-\frac{\tilde{\sigma}}{|\epsilon|} r_{b}(\beta)|\tau|-\exp \left(\tilde{\sigma}^{\prime} r_{b}(\beta)|\tau|\right)\right)
\end{aligned}
$$

for all $\tau \in S_{d} \cup D(0, r)$. From this latter equality, we get

$$
\left\|\exp \left(-k_{2} \tau\right) \tau^{k_{0}} v_{\beta}(\tau)\right\|_{\beta, \epsilon, \sigma, \sigma^{\prime}, \Omega} \leq B(\epsilon, \beta)\left\|v_{\beta}(\tau)\right\|_{\beta, \epsilon, \tilde{\sigma}, \tilde{\sigma}^{\prime}, \Omega}
$$

where

$$
\begin{aligned}
B(\epsilon, \beta)=\sup _{\tau \in S_{d} \cup D(0, r)} \exp \left(k_{2}|\tau|\right)|\tau|^{k_{0}} \exp \left(\frac{\tilde{\sigma}}{|\epsilon|} r_{b}(\beta)|\tau|\right. & \left.+\exp \left(\tilde{\sigma}^{\prime} r_{b}(\beta)|\tau|\right)\right) \\
& \times \exp \left(-\frac{\sigma}{|\epsilon|} r_{b}(\beta)|\tau|-\exp \left(\sigma^{\prime} r_{b}(\beta)|\tau|\right)\right)
\end{aligned}
$$

for all $\beta \geq 0$. Moreover, using the estimates (25), we deduce that

$$
\sup _{\tau \in S_{d} \cup D(0, r)}|\tau|^{k_{0}} \exp \left(-\frac{(\sigma-\tilde{\sigma}) r_{b}(\beta)}{|\epsilon|}|\tau|\right) \leq|\epsilon|^{k_{0}}\left(\frac{k_{0} e^{-1}}{\sigma-\tilde{\sigma}}\right)^{k_{0}}
$$

for all $\beta \geq 0$.

On the other hand, there exists a constant $\tilde{d}>0$ (depending on $\left.k_{2}, \sigma^{\prime}, \tilde{\sigma}^{\prime}\right)$ such that

$$
\exp \left(k_{2}|\tau|+\exp \left(\tilde{\sigma}^{\prime} r_{b}(\beta)|\tau|\right)-\exp \left(\sigma^{\prime} r_{b}(\beta)|\tau|\right)\right) \leq \exp (\tilde{d})
$$

for all $\beta \geq 0$, all $\tau \in S_{d} \cup D(0, r)$. Indeed, from the Taylor formula, we know that for all $\tau \in S_{d} \cup D(0, r)$, all $\beta \geq 0$ there exists a constant $c \in\left(\tilde{\sigma}^{\prime} r_{b}(\beta)|\tau|, \sigma^{\prime} r_{b}(\beta)|\tau|\right)$ such that

$$
\begin{aligned}
\exp \left(\tilde{\sigma}^{\prime} r_{b}(\beta)|\tau|\right)-\exp \left(\sigma^{\prime} r_{b}(\beta)|\tau|\right) & \\
& =\left(\tilde{\sigma}^{\prime}-\sigma^{\prime}\right) r_{b}(\beta)|\tau| e^{c} \leq-\left(\sigma^{\prime}-\tilde{\sigma}^{\prime}\right) r_{b}(\beta)|\tau| \exp \left(\tilde{\sigma}^{\prime} r_{b}(\beta)|\tau|\right)
\end{aligned}
$$

From (28) we deduce that

$$
\begin{aligned}
\sup _{\tau \in S_{d} \cup D(0, r)} k_{2}|\tau|-\left(\sigma^{\prime}-\tilde{\sigma}^{\prime}\right) r_{b}(\beta)|\tau| & \exp \left(\tilde{\sigma}^{\prime} r_{b}(\beta)|\tau|\right) \\
& \leq \max \left(k_{2}, \frac{k_{2}}{\tilde{\sigma}^{\prime} r_{b}(\beta)}\left(\log \left(\frac{k_{2}}{\left(\sigma^{\prime}-\tilde{\sigma}^{\prime}\right) \tilde{\sigma}^{\prime}\left(r_{b}(\beta)\right)^{2}}\right)-1\right)\right)
\end{aligned}
$$


for all $\beta \geq 0$. From (46) and (47), one deduces (45). Finally, from (44) and (45), one obtains a constant $\tilde{C}_{1}>0$ (depending on $k_{0}, k_{2}, \sigma, \tilde{\sigma}, \sigma^{\prime}, \tilde{\sigma}^{\prime}$ ) such that $B(\epsilon, \beta) \leq \tilde{C}_{1}|\epsilon|^{k_{0}}$ for all $\beta \geq 0$. Hence, the lemma 5 follows.

The estimates (38) result from (40) and (41).

2) Let $v(\tau, z) \in S G(\epsilon, \sigma, 0, \delta, \Omega)$. By definition, we have

$$
\left\|\tau^{k_{0}} v(\tau, z)\right\|_{(\epsilon, \sigma, 0, \delta, \Omega)}=\sum_{\beta \geq 0}\left\|\tau^{k_{0}} v_{\beta}(\tau)\right\|_{\beta, \epsilon, \sigma, 0, \Omega} \frac{\delta^{\beta}}{\beta !} .
$$

Lemma 6 There exists a constant $\tilde{C}_{1.1}>0$ (depending on $\left.k_{0}, \sigma, \tilde{\sigma}\right)$ such that

$$
\left\|\tau^{k_{0}} v_{\beta}(\tau)\right\|_{\beta, \epsilon, \sigma, 0, \Omega} \leq \tilde{C}_{1.1}|\epsilon|^{k_{0}}\left\|v_{\beta}(\tau)\right\|_{\beta, \epsilon, \tilde{\sigma}, 0, \Omega}
$$

for all $\beta \geq 0$.

Proof The arguments relies on the beginning of Lemma 5 . If one puts $\sigma^{\prime}=0, \tilde{\sigma}^{\prime}=0$ and $k_{2}=0$ in (42), one gets

$$
\left\|\tau^{k_{0}} v_{\beta}(\tau)\right\|_{\beta, \epsilon, \sigma, 0, \Omega} \leq \tilde{B}(\epsilon, \beta)\left\|v_{\beta}(\tau)\right\|_{\beta, \epsilon, \tilde{\sigma}, 0, \Omega}
$$

where

$$
\tilde{B}(\epsilon, \beta)=\sup _{\tau \in S_{d} \cup D(0, r)}|\tau|^{k_{0}} \exp \left(\frac{\tilde{\sigma}}{|\epsilon|} r_{b}(\beta)|\tau|\right) \times \exp \left(-\frac{\sigma}{|\epsilon|} r_{b}(\beta)|\tau|\right)
$$

for all $\beta \geq 0$. Gathering (50) and (44) yields Lemma 6 .

The estimates (39) follow from (48) and (49).

In the next proposition, we study linear operators of multiplication by bounded holomorphic functions.

Proposition 4 Let $h(\tau, z, \epsilon)$ be a holomorphic function on $\left(S_{d} \cup D(0, r)\right) \times D(0, \rho) \times \mathcal{E}$, for some $\rho>0$, bounded by some constant $M>0$. Let $0<\delta<\rho$. Then, the linear operator of multiplication by $h(\tau, z, \epsilon)$ is continuous from $\left(S G\left(\epsilon, \sigma, \sigma^{\prime}, \delta, \Omega\right),\|\cdot\|_{\left(\epsilon, \sigma, \sigma^{\prime}, \delta, \Omega\right)}\right)$ into itself, for all $\epsilon \in \mathcal{E}$. Moreover, there exists a constant $C_{2}$ (depending on $M, \delta, \rho$ ), independent of $\epsilon$, such that

$$
\|h(\tau, z, \epsilon) v(\tau, z)\|_{\left(\epsilon, \sigma, \sigma^{\prime}, \delta, \Omega\right)} \leq C_{2}\|v(\tau, z)\|_{\left(\epsilon, \sigma, \sigma^{\prime}, \delta, \Omega\right)}
$$

for all $v(\tau, z) \in S G\left(\epsilon, \sigma, \sigma^{\prime}, \delta, \Omega\right)$, for all $\epsilon \in \mathcal{E}$.

Proof Let $h(\tau, z, \epsilon)=\sum_{\beta \geq 0} h_{\beta}(\tau, \epsilon) z^{\beta} / \beta$ ! be holomorphic on $\left(S_{d} \cup D(0, r)\right) \times D(0, \rho) \times \mathcal{E}$ such that there exists $M>0$ with

$$
\sup _{\tau \in S_{d} \cup D(0, r), z \in D(0, \rho), \epsilon \in \mathcal{E}}|h(\tau, z, \epsilon)| \leq M .
$$

Let $v(\tau, z)=\sum_{\beta \geq 0} v_{\beta}(\tau) z^{\beta} / \beta ! \in S G\left(\epsilon, \sigma, \sigma^{\prime}, \delta, \Omega\right)$. By construction, we have that

$$
\|h(\tau, z, \epsilon) v(\tau, z)\|_{\left(\epsilon, \sigma, \sigma^{\prime}, \delta, \Omega\right)} \leq \sum_{\beta \geq 0}\left(\sum_{\beta_{1}+\beta_{2}=\beta}\left\|h_{\beta_{1}}(\tau, \epsilon) v_{\beta_{2}}(\tau)\right\|_{\beta, \epsilon, \sigma, \sigma^{\prime}, \Omega} \frac{\beta !}{\beta_{1} ! \beta_{2} !}\right) \frac{\delta^{\beta}}{\beta !} .
$$


From the Cauchy formula, we have

$$
\sup _{\tau \in S_{d} \cup D(0, r), \epsilon \in \mathcal{E}}\left|h_{\beta}(\tau, \epsilon)\right| \leq M\left(\frac{1}{\delta^{\prime}}\right)^{\beta} \beta !
$$

for any $\delta<\delta^{\prime}<\rho$, for all $\beta \geq 0$. By definition, we deduce that

(54) $\left\|h_{\beta_{1}}(\tau, \epsilon) v_{\beta_{2}}(\tau)\right\|_{\beta, \epsilon, \sigma, \sigma^{\prime}, \Omega} \leq M \beta_{1} !\left(\frac{1}{\delta^{\prime}}\right)^{\beta_{1}}\left\|v_{\beta_{2}}(\tau)\right\|_{\beta, \epsilon, \sigma, \sigma^{\prime}, \Omega} \leq M \beta_{1} !\left(\frac{1}{\delta^{\prime}}\right)^{\beta_{1}}\left\|v_{\beta_{2}}(\tau)\right\|_{\beta_{2}, \epsilon, \sigma, \sigma^{\prime}, \Omega}$ for all $\beta_{1}, \beta_{2} \geq 0$ such that $\beta_{1}+\beta_{2}=\beta$. From (53) and (54), we deduce that

$$
\|h(\tau, z, \epsilon) v(\tau, z)\|_{\left(\epsilon, \sigma, \sigma^{\prime}, \delta, \Omega\right)} \leq M\left(\sum_{\beta \geq 0}\left(\frac{\delta}{\delta^{\prime}}\right)^{\beta}\right)\|v(\tau, z)\|_{\left(\epsilon, \sigma, \sigma^{\prime}, \delta, \Omega\right)}
$$

which yields (52).

In the next proposition, we give norm estimates for the convolution product.

Proposition 5 Let $f, g$ be in $S G\left(\epsilon, \sigma, \sigma^{\prime}, \delta, \Omega\right)$. Then, the function

$$
(f * g)(\tau, z)=\int_{0}^{\tau} f(\tau-s, z) g(s, z) d s
$$

belongs to $S G\left(\epsilon, \sigma, \sigma^{\prime}, \delta, \Omega\right)$. Moreover, there exists a (universal) constant $C_{3}>0$ such that

$$
\|(f * g)(\tau, z)\|_{\left(\epsilon, \sigma, \sigma^{\prime}, \delta, \Omega\right)} \leq C_{3}|\epsilon|\|f(\tau, z)\|_{\left(\epsilon, \sigma, \sigma^{\prime}, \delta, \Omega\right)}\|g(\tau, z)\|_{\left(\epsilon, \sigma, \sigma^{\prime}, \delta, \Omega\right)}
$$

for all $f, g \in S G\left(\epsilon, \sigma, \sigma^{\prime}, \delta, \Omega\right)$.

\section{Proof Let}

$$
f(\tau, z)=\sum_{\beta \geq 0} f_{\beta}(\tau) z^{\beta} / \beta !, g(\tau, z)=\sum_{\beta \geq 0} g_{\beta}(\tau) z^{\beta} / \beta !
$$

be in $S G\left(\epsilon, \sigma, \sigma^{\prime}, \delta, \Omega\right)$. By construction of $f * g$, we have that

$$
\begin{aligned}
& \left\|\int_{0}^{\tau} f(\tau-s, z) g(s, z) d s\right\|_{\left(\epsilon, \sigma, \sigma^{\prime}, \delta, \Omega\right)} \\
& \quad \leq \sum_{\beta \geq 0}\left(\sum_{\beta_{1}+\beta_{2}=\beta} \frac{\beta !}{\beta_{1} ! \beta_{2} !}\left\|\int_{0}^{\tau} f_{\beta_{1}}(\tau-s) g_{\beta_{2}}(s) d s\right\|_{\beta, \epsilon, \sigma, \sigma^{\prime}, \Omega}\right) \frac{\delta^{\beta}}{\beta !} .
\end{aligned}
$$

Lemma 7 There exists a (universal) constant $C_{3}>0$ such that

$$
\left\|\int_{0}^{\tau} f_{\beta_{1}}(\tau-s) g_{\beta_{2}}(s) d s\right\|\left\|_{\beta, \epsilon, \sigma, \sigma^{\prime}, \Omega} \leq C_{3}|\epsilon|\right\| f_{\beta_{1}}(\tau)\left\|_{\beta_{1}, \epsilon, \sigma, \sigma^{\prime}, \Omega}\right\| g_{\beta_{2}}(\tau) \|_{\beta_{2}, \epsilon, \sigma, \sigma^{\prime}, \Omega}
$$

for all $\beta \geq 0$ and all $\beta_{1}, \beta_{2} \geq 0$ with $\beta_{1}+\beta_{2}=\beta$.

Proof We write

$$
\begin{gathered}
\left|\int_{0}^{\tau} f_{\beta_{1}}(\tau-s) g_{\beta_{2}}(s) d s\right|=\mid \int_{0}^{\tau} f_{\beta_{1}}(\tau-s)\left(1+\frac{|\tau-s|^{2}}{|\epsilon|^{2}}\right) \\
\times \exp \left(-\frac{\sigma}{|\epsilon|} r_{b}\left(\beta_{1}\right)|\tau-s|-\exp \left(\sigma^{\prime} r_{b}\left(\beta_{1}\right)|\tau-s|\right)\right) \\
\times g_{\beta_{2}}(s)\left(1+\frac{|s|^{2}}{|\epsilon|^{2}}\right) \exp \left(-\frac{\sigma}{|\epsilon|} r_{b}\left(\beta_{2}\right)|s|-\exp \left(\sigma^{\prime} r_{b}\left(\beta_{2}\right)|s|\right)\right) \\
\times \frac{\exp \left(\frac{\sigma}{|\epsilon|}\left(r_{b}\left(\beta_{1}\right)|\tau-s|+r_{b}\left(\beta_{2}\right)|s|\right)+\exp \left(\sigma^{\prime} r_{b}\left(\beta_{1}\right)|\tau-s|\right)+\exp \left(\sigma^{\prime} r_{b}\left(\beta_{2}\right)|s|\right)\right)}{\left(1+\frac{|s|^{2}}{|\epsilon|^{2}}\right)\left(1+\frac{|\tau-s|^{2}}{|\epsilon|^{2}}\right)} d s \mid
\end{gathered}
$$


for all $\tau \in S_{d} \cup D(0, r)$. We deduce that

$\left|\int_{0}^{\tau} f_{\beta_{1}}(\tau-s) g_{\beta_{2}}(s) d s\right| \leq\left\|f_{\beta_{1}}(\tau)\right\|_{\beta_{1}, \epsilon, \sigma, \sigma^{\prime}, \Omega}\left\|g_{\beta_{2}}(\tau)\right\|_{\beta_{2}, \epsilon, \sigma, \sigma^{\prime}, \Omega}$

$\times \int_{0}^{1} \frac{|\tau| \exp \left(\frac{\sigma|\tau|}{|\epsilon|}\left(r_{b}\left(\beta_{1}\right)(1-h)+r_{b}\left(\beta_{2}\right) h\right)+\exp \left(\sigma^{\prime} r_{b}\left(\beta_{1}\right)|\tau|(1-h)\right)+\exp \left(\sigma^{\prime} r_{b}\left(\beta_{2}\right)|\tau| h\right)\right)}{\left(1+\frac{|\tau|^{2}}{|\epsilon|^{2}}(1-h)^{2}\right)\left(1+\frac{|\tau|^{2}}{|\epsilon|^{2}} h^{2}\right)} d h$.

In the next step we will show that there exists a constant $C_{3}>0$ such that

$$
\begin{aligned}
& I\left(|\tau|,|\epsilon|, \beta, \beta_{1}, \beta_{2}\right)=\left(1+\frac{|\tau|^{2}}{|\epsilon|^{2}}\right) \exp \left(-\frac{\sigma}{|\epsilon|} r_{b}(\beta)|\tau|-\exp \left(\sigma^{\prime} r_{b}(\beta)|\tau|\right)\right) \\
& \times \int_{0}^{1} \frac{|\tau| \exp \left(\frac{\sigma|\tau|}{|\epsilon|}\left(r_{b}\left(\beta_{1}\right)(1-h)+r_{b}\left(\beta_{2}\right) h\right)+\exp \left(\sigma^{\prime} r_{b}\left(\beta_{1}\right)|\tau|(1-h)\right)+\exp \left(\sigma^{\prime} r_{b}\left(\beta_{2}\right)|\tau| h\right)\right)}{\left(1+\frac{|\tau|^{2}}{|\epsilon|^{2}}(1-h)^{2}\right)\left(1+\frac{|\tau|^{2}}{|\epsilon|^{2}} h^{2}\right)} d h \\
& \leq|\epsilon| C_{3}
\end{aligned}
$$

for all $\tau \in S_{d} \cup D(0, r)$, all $\epsilon \in \mathcal{E}$, for all $\beta \geq 0$, all $\beta_{1}, \beta_{2} \geq 0$ with $\beta_{1}+\beta_{2}=\beta$. Indeed, from the fact that $r_{b}$ is increasing, we first have that

$$
r_{b}\left(\beta_{1}\right)(1-h)+r_{b}\left(\beta_{2}\right) h \leq r_{b}(\beta)
$$

for all $0 \leq h \leq 1$, all $\beta_{1}, \beta_{2} \geq 0$ with $\beta_{1}+\beta_{2}=\beta$. Then, from (60), we get that

$$
\exp \left(-\frac{\sigma}{|\epsilon|} r_{b}(\beta)|\tau|\right) \exp \left(\frac{\sigma|\tau|}{|\epsilon|}\left(r_{b}\left(\beta_{1}\right)(1-h)+r_{b}\left(\beta_{2}\right) h\right)\right) \leq 1
$$

for all $\tau \in S_{d} \cup D(0, r)$, all $\epsilon \in \mathcal{E}$, for all $\beta \geq 0$, all $\beta_{1}, \beta_{2} \geq 0$ with $\beta_{1}+\beta_{2}=\beta$ and all $h \in[0,1]$.

On the other hand, since $r_{b}$ is increasing, we get that

$$
\begin{aligned}
& \exp \left(\sigma^{\prime} r_{b}\left(\beta_{1}\right)|\tau|(1-h)\right)+\exp \left(\sigma^{\prime} r_{b}\left(\beta_{2}\right)|\tau| h\right)-\exp \left(\sigma^{\prime} r_{b}(\beta)|\tau|\right) \\
& \leq \exp \left(\sigma^{\prime} r_{b}(\beta)|\tau|(1-h)\right)+\exp \left(\sigma^{\prime} r_{b}(\beta)|\tau| h\right)-\exp \left(\sigma^{\prime} r_{b}(\beta)|\tau|\right)=\varphi(h)
\end{aligned}
$$

for all $h \in[0,1]$, for all $\tau \in S_{d} \cup D(0, r)$, all $\epsilon \in \mathcal{E}$, for all $\beta \geq 0$, all $\beta_{1}, \beta_{2} \geq 0$ with $\beta_{1}+\beta_{2}=\beta$. By construction, one has $\varphi(0)=\varphi(1)=1$. Moreover, by direct computation, one can check that $\varphi^{\prime}(h) \leq 0$ if $0 \leq h \leq 1 / 2$ and $\varphi^{\prime}(h) \geq 0$ if $1 / 2 \leq h \leq 1$. So that $\varphi(h) \leq 1$ for all $h \in[0,1]$.

From (61) and (62), we deduce that

$$
I\left(|\tau|,|\epsilon|, \beta, \beta_{1}, \beta_{2}\right) \leq J(|\tau|,|\epsilon|)=\int_{0}^{1} \frac{e\left(1+\frac{|\tau|^{2}}{|\epsilon|^{2}}\right)|\tau|}{\left(1+\frac{|\tau|^{2}}{|\epsilon|^{2}}(1-h)^{2}\right)\left(1+\frac{|\tau|^{2}}{|\epsilon|^{2}} h^{2}\right)} d h
$$

for all $\tau \in S_{d} \cup D(0, r)$, all $\epsilon \in \mathcal{E}$. On the other hand, we have that

$$
\frac{J(|\epsilon||\tau|,|\epsilon|)}{|\epsilon|}=\int_{0}^{1} \frac{e\left(1+|\tau|^{2}\right)|\tau|}{\left(1+|\tau|^{2}(1-h)^{2}\right)\left(1+|\tau|^{2} h^{2}\right)} d h .
$$

From Corollary 4.9 of [10], we know that the right hand side of (64) is a bounded function of $|\tau|$ on $\mathbb{R}_{+}$. We deduce that there exists a (universal) constant $C_{3}>0$ such that

$$
\sup _{|\tau| \geq 0} \frac{J(|\tau|,|\epsilon|)}{|\epsilon|}=\sup _{|\tau| \geq 0} \frac{J(|\epsilon||\tau|,|\epsilon|)}{|\epsilon|} \leq C_{3}
$$


for all $\epsilon \in \mathcal{E}$. We get from (63) and (65) that the inequality (59) holds. Finally, the inequality (57) follows from (58) and (59).

From (56) and (57), we get that (55) holds with the constant $C_{3}$ from Lemma 7.

Corollary 1 Let $s, k_{0} \geq 0$ be non negative integers and let $k_{1}, k_{2} \in \mathbb{N}^{*}$.

1) Let $\sigma^{\prime}>0$. Assume that the condition

$$
k_{1} \geq b k_{0}+\frac{k_{2} b}{\sigma^{\prime}}
$$

holds. Moreover, we assume that the function $\tau \mapsto \exp \left(-k_{2} \tau\right)$ is unbounded on the sector $S_{d}$. Then, for all $\epsilon \in \mathcal{E}$, the operator

$$
v(\tau, z) \mapsto \int_{0}^{\tau}(\tau-h)^{s} \exp \left(-k_{2} h\right) h^{k_{0}} \partial_{z}^{-k_{1}} v(h, z) d h
$$

is a bounded linear operator from $\left(S G\left(\epsilon, \sigma, \sigma^{\prime}, \delta, \Omega\right),\|\cdot\|_{\left(\epsilon, \sigma, \sigma^{\prime}, \delta, \Omega\right)}\right)$ into itself. Moreover, there exists a constant $C_{4}>0$ (depending on $s, k_{0}, k_{1}, k_{2}, \sigma, \sigma^{\prime}, b$ ), which does not depend on $\epsilon \in \mathcal{E}$, such that

$$
\left\|\int_{0}^{\tau}(\tau-h)^{s} \exp \left(-k_{2} h\right) h^{k_{0}} \partial_{z}^{-k_{1}} v(h, z) d h\right\|_{\left(\epsilon, \sigma, \sigma^{\prime}, \delta, \Omega\right)} \leq C_{4}|\epsilon|^{s+k_{0}+1} \delta^{k_{1}}\|v(\tau, z)\|_{\left(\epsilon, \sigma, \sigma^{\prime}, \delta, \Omega\right)}
$$

for all $v \in S G\left(\epsilon, \sigma, \sigma^{\prime}, \delta, \Omega\right)$, all $\epsilon \in \mathcal{E}$.

2) Assume that the condition

$$
k_{1} \geq b k_{0}
$$

holds. Let $q(\tau)$ be a holomorphic function bounded by some constant $M>0$ on $S_{d} \cup D(0, r)$. Then, for all $\epsilon \in \mathcal{E}$, the operator

$$
v(\tau, z) \mapsto \int_{0}^{\tau}(\tau-h)^{s} q(h) h^{k_{0}} \partial_{z}^{-k_{1}} v(h, z) d h
$$

is a bounded linear operator from $\left(S G(\epsilon, \sigma, 0, \delta, \Omega),\|\cdot\|_{(\epsilon, \sigma, 0, \delta, \Omega)}\right)$ into itself. Moreover, there exists a constant $C_{4.1}>0$ (depending on $s, k_{0}, k_{1}, \sigma, b, M$ ), which does not depend on $\epsilon \in \mathcal{E}$, such that

$$
\left\|\int_{0}^{\tau}(\tau-h)^{s} q(h) h^{k_{0}} \partial_{z}^{-k_{1}} v(h, z) d h\right\|_{(\epsilon, \sigma, 0, \delta, \Omega)} \leq C_{4.1}|\epsilon|^{s+k_{0}+1} \delta^{k_{1}}\|v(\tau, z)\|_{(\epsilon, \sigma, 0, \delta, \Omega)}
$$

for all $v \in S G(\epsilon, \sigma, 0, \delta, \Omega)$, all $\epsilon \in \mathcal{E}$.

Proof 1) Using Proposition 5, there exists a universal constant $C_{3}>0$ for which

$$
\begin{aligned}
\left\|\int_{0}^{\tau}(\tau-h)^{s} \exp \left(-k_{2} h\right) h^{k_{0}} \partial_{z}^{-k_{1}} v(h, z) d h\right\|_{\left(\epsilon, \sigma, \sigma^{\prime}, \delta, \Omega\right)} \\
\quad \leq C_{3}|\epsilon|\left\|\tau^{s}\right\|_{\left(\epsilon, \sigma, \sigma^{\prime}, \delta, \Omega\right)}\left\|\exp \left(-k_{2} \tau\right) \tau^{k_{0}} \partial_{z}^{-k_{1}} v(\tau, z)\right\|_{\left(\epsilon, \sigma, \sigma^{\prime}, \delta, \Omega\right)}
\end{aligned}
$$


holds. Moreover, using the estimates (25) we notice that

$$
\left\|\tau^{s}\right\|_{\left(\epsilon, \sigma, \sigma^{\prime}, \delta, \Omega\right)}=\left\|\tau^{s}\right\|_{0, \epsilon, \sigma, \sigma^{\prime}, \Omega} \leq\left\|\tau^{s}\right\|_{0, \epsilon, \sigma, 0, \Omega}=|\epsilon|^{s}\left(\left(\frac{s e^{-1}}{\sigma}\right)^{s}+\left(\frac{(s+2) e^{-1}}{\sigma}\right)^{s+2}\right) .
$$

Finally, gathering (15), (70) and (71) yields (67).

2) Again, due to Proposition 5, we get a universal constant $C_{3}>0$ such that

$$
\begin{aligned}
& \left\|\int_{0}^{\tau}(\tau-h)^{s} q(h) h^{k_{0}} \partial_{z}^{-k_{1}} v(h, z) d h\right\|_{(\epsilon, \sigma, 0, \delta, \Omega)} \\
& \quad \leq C_{3}|\epsilon|\left\|\tau^{s}\right\|_{(\epsilon, \sigma, 0, \delta, \Omega)}\left\|q(\tau) \tau^{k_{0}} \partial_{z}^{-k_{1}} v(\tau, z)\right\|_{(\epsilon, \sigma, 0, \delta, \Omega)}
\end{aligned}
$$

Using (17) and (52) together with (71) and (72) give the result.

\subsection{A global Cauchy problem}

We keep the same notations as in the previous section. In the following, we introduce some definitions. Let $\mathcal{A}_{1}$ (resp. $\mathcal{A}_{2}$ ) be a finite subset of $\mathbb{N}^{4}$ (resp. $\mathbb{N}$ ).

For all $\underline{k}=\left(s, k_{0}, k_{1}, k_{2}\right) \in \mathcal{A}_{1}$, we denote $I_{\underline{k}}$ a finite subset of $\mathbb{N}$. For all $n \in I_{\underline{k}}$, we denote $a_{n, \underline{k}}(\tau, z, \epsilon)$ some bounded holomorphic function on $\left(S_{d} \cup D(0, r)\right) \times D(0, \rho) \times \mathcal{E}$, for some $\rho>0$. For all $\underline{k} \in \mathcal{A}_{1}$, we consider

$$
a_{\underline{k}}(\tau, z, \epsilon)=\sum_{n \in I_{\underline{k}}} a_{n, \underline{k}}(\tau, z, \epsilon) \epsilon^{-n}
$$

which are holomorphic functions on $\left(S_{d} \cup D(0, r)\right) \times D(0, \rho) \times \mathcal{E}$. For all $l \in \mathcal{A}_{2}$, we denote $\alpha_{l}(\tau, z, \epsilon)$ some bounded holomorphic function on $\left(S_{d} \cup D(0, r)\right) \times D(0, \rho) \times \mathcal{E}$.

Let $S \geq 1$ be an integer. We consider the following equation

$$
\begin{aligned}
\partial_{z}^{S} V(\tau, z, \epsilon)=\sum_{\underline{k} \in \mathcal{A}_{1}} a_{\underline{k}}(\tau, z, \epsilon) \int_{0}^{\tau}(\tau-h)^{s} \exp \left(-k_{2} h\right) h^{k_{0}} \partial_{z}^{k_{1}} V(h, z, \epsilon) d h & \\
& +\sum_{l \in \mathcal{A}_{2}} \alpha_{l}(\tau, z, \epsilon) V^{* l}(\tau, z, \epsilon)
\end{aligned}
$$

where $V^{* 1}=V$ and $V^{* l_{1}}, l_{1} \geq 2$, stands for the convolution product of $V$ applied $l_{1}-1$ times with respect to $\tau$.

We state the main result of this section.

Proposition 6 1) We make the following assumptions.

There exist real numbers $\sigma^{\prime}>\tilde{\sigma}^{\prime}>0$ such that, for all $\underline{k} \in \mathcal{A}_{1}$, all $n \in I_{\underline{k}}$, we have

$$
S \geq k_{1}+b k_{0}+\frac{k_{2} b}{\sigma^{\prime}}, \quad s+k_{0}+1 \geq n \quad, \quad S>k_{1} .
$$

For all $l \in \mathcal{A}_{2}$, we have

$$
l \geq 2
$$

For all $0 \leq j \leq S-1$, we consider a function $\tau \mapsto V_{j}(\tau, \epsilon)$ that belongs to $S E_{0, \epsilon, \tilde{\sigma}, \tilde{\sigma}^{\prime}, \Omega}$, for some $\tilde{\sigma}>0$ and all $\epsilon \in \mathcal{E}$. We assume that the function $\tau \mapsto \exp \left(-k_{2} \tau\right)$ is unbounded on the sector $S_{d}$ 
Then, there exist constants $I>0, R>0$ and $\delta>0$ (independent of $\epsilon$ ) such that if we assume that

$$
\sum_{j=0}^{S-1-h}\left\|V_{j+h}(\tau, \epsilon)\right\|_{0, \epsilon, \tilde{\sigma}, \tilde{\sigma}^{\prime}, \Omega} \frac{\delta^{j}}{j !} \leq I,
$$

for all $0 \leq h \leq S-1$, for all $\epsilon \in \mathcal{E}$, the equation (73) with initial data

$$
\left(\partial_{z}^{j} V\right)(\tau, 0, \epsilon)=V_{j}(\tau, \epsilon) \quad, \quad 0 \leq j \leq S-1,
$$

has a unique solution $V(\tau, z, \epsilon)$ in the space $S G\left(\epsilon, \sigma, \sigma^{\prime}, \delta, \Omega\right)$, for some $\sigma>\tilde{\sigma}$, for all $\epsilon \in \mathcal{E}$, which satisfies moreover the estimates

$$
\|V(\tau, z, \epsilon)\|_{\left(\epsilon, \sigma, \sigma^{\prime}, \delta, \Omega\right)} \leq \delta^{S} R+I
$$

for all $\epsilon \in \mathcal{E}$.

2) We assume that the next conditions hold.

For all $\underline{k} \in \mathcal{A}_{1}$, all $n \in I_{\underline{k}}$,

$$
S \geq k_{1}+b k_{0} \quad, \quad s+k_{0}+1 \geq n \quad, \quad S>k_{1}
$$

hold and for all $l \in \mathcal{A}_{2}$, we have

$$
l \geq 2
$$

For all $0 \leq j \leq S-1$, we consider a function $\tau \mapsto V_{j}(\tau, \epsilon)$ that belongs to $S E_{0, \epsilon, \tilde{\sigma}, 0, \Omega}$, for some $\tilde{\sigma}>0$ and all $\epsilon \in \mathcal{E}$. We make the assumption that the function $\tau \mapsto \exp \left(-k_{2} \tau\right)$ is bounded on the sector $S_{d}$.

Then, there exist constants $I>0, R>0$ and $\delta>0$ (independent of $\epsilon$ ) such that if we assume that

$$
\sum_{j=0}^{S-1-h}\left\|V_{j+h}(\tau, \epsilon)\right\|_{0, \epsilon, \tilde{\sigma}, 0, \Omega} \frac{\delta^{j}}{j !} \leq I,
$$

for all $0 \leq h \leq S-1$, for all $\epsilon \in \mathcal{E}$, the equation (73) with initial data

$$
\left(\partial_{z}^{j} V\right)(\tau, 0, \epsilon)=V_{j}(\tau, \epsilon) \quad, \quad 0 \leq j \leq S-1,
$$

has a unique solution $V(\tau, z, \epsilon)$ in the space $S G(\epsilon, \sigma, 0, \delta, \Omega)$, for some $\sigma>\tilde{\sigma}$, for all $\epsilon \in \mathcal{E}$, which satisfies moreover the estimates

$$
\|V(\tau, z, \epsilon)\|_{(\epsilon, \sigma, 0, \delta, \Omega)} \leq \delta^{S} R+I,
$$

for all $\epsilon \in \mathcal{E}$.

Proof We consider

$$
w(\tau, z, \epsilon)=\sum_{j=0}^{S-1} V_{j}(\tau, \epsilon) \frac{z^{j}}{j !}
$$


where $V_{j}(\tau, \epsilon)$ are given in (77) or (81). For all $\epsilon \in \mathcal{E}$, we define a map $A_{\epsilon}$ from $\mathcal{O}\left(S_{d} \cup D(0, r)\right)\{z\}$ into itself by

$$
\begin{aligned}
A_{\epsilon}(U(\tau, z))=\sum_{\underline{k} \in \mathcal{A}_{1}} a_{\underline{k}}(\tau, z, \epsilon) \int_{0}^{\tau}(\tau-h)^{s} \exp \left(-k_{2} h\right) h^{k_{0}} \partial_{z}^{k_{1}-S} U(h, z) d h \\
+\sum_{\underline{k} \in \mathcal{A}_{1}} a_{\underline{k}}(\tau, z, \epsilon) \int_{0}^{\tau}(\tau-h)^{s} \exp \left(-k_{2} h\right) h^{k_{0}} \partial_{z}^{k_{1}} w(h, z, \epsilon) d h \\
+\sum_{l \in \mathcal{A}_{2}} \alpha_{l}(\tau, z, \epsilon)\left(\left(\partial_{z}^{-S} U(\tau, z)+w(\tau, z, \epsilon)\right)^{* l_{1}}\right)
\end{aligned}
$$

In the following, we only plan to give details for the point 1) since exactly the same lines of arguments apply for the point 2) of the Proposition 6 with the help of the point 2) of Propositions 2,3 and Corollary 1 instead.

In the next lemma, we show that $A_{\epsilon}$ is a Lipschitz shrinking map from and into a small ball in a neighborhood of the origin of $S G\left(\epsilon, \sigma, \sigma^{\prime}, \delta, \Omega\right)$, for some $\sigma>\tilde{\sigma}, \sigma^{\prime}>\tilde{\sigma}^{\prime}$.

Lemma 8 Under the conditions (74), (75), let a real number I be such that

$$
\sum_{j=0}^{S-1-h}\left\|V_{j+h}(\tau, \epsilon)\right\|_{0, \epsilon, \tilde{\sigma}, \tilde{\sigma}^{\prime}, \Omega} \frac{\delta^{j}}{j !} \leq I,
$$

for all $0 \leq h \leq S-1$, for all $\epsilon \in \mathcal{E}$. Then, for a good choice of $I>0$,

a) there exist real numbers $0<\delta<\rho, \sigma>\tilde{\sigma}, \sigma^{\prime}>\tilde{\sigma}^{\prime}$ and $R>0$ (not depending on $\epsilon$ ) such that

$$
\left\|A_{\epsilon}(U(\tau, z))\right\|_{\left(\epsilon, \sigma, \sigma^{\prime}, \delta, \Omega\right)} \leq R
$$

for all $U(\tau, z) \in B(0, R)$, for all $\epsilon \in \mathcal{E}$, where $B(0, R)$ is the closed ball centered at 0 with radius $R$ in $S G\left(\epsilon, \sigma, \sigma^{\prime}, \delta, \Omega\right)$,

b) we have

$$
\left\|A_{\epsilon}\left(U_{1}(\tau, z)\right)-A_{\epsilon}\left(U_{2}(\tau, z)\right)\right\|_{\left(\epsilon, \sigma, \sigma^{\prime}, \delta, \Omega\right)} \leq \frac{1}{2}\left\|U_{1}(\tau, z)-U_{2}(\tau, z)\right\|_{\left(\epsilon, \sigma, \sigma^{\prime}, \delta, \Omega\right)}
$$

for all $U_{1}, U_{2} \in B(0, R)$, for all $\epsilon \in \mathcal{E}$.

Proof First of all, for all $0 \leq h \leq S-1,0 \leq j \leq S-1-h$, we have that

$$
\left\|V_{j+h}(\tau, \epsilon)\right\|_{j, \epsilon, \tilde{\sigma}, \tilde{\sigma}^{\prime}, \Omega} \leq\left\|V_{j+h}(\tau, \epsilon)\right\|_{0, \epsilon, \tilde{\sigma}, \tilde{\sigma}^{\prime}, \Omega} .
$$

We deduce that $\partial_{z}^{h} w(\tau, z, \epsilon) \in S G\left(\epsilon, \tilde{\sigma}, \tilde{\sigma}^{\prime}, \delta, \Omega\right)$ and that

$$
\left\|\partial_{z}^{h} w(\tau, z, \epsilon)\right\|_{\left(\epsilon, \tilde{\sigma}, \tilde{\sigma}^{\prime}, \delta, \Omega\right)} \leq \sum_{j=0}^{S-1-h}\left\|V_{j+h}(\tau, \epsilon)\right\|_{0, \epsilon, \tilde{\sigma}, \tilde{\sigma}^{\prime}, \Omega} \frac{\delta^{j}}{j !} \leq I
$$

for all $0 \leq h \leq S-1$.

We first show the estimates (82). 
Let $\sigma>\tilde{\sigma}, \sigma^{\prime}>\tilde{\sigma}^{\prime}, R>0$ and $U(\tau, z) \in S G\left(\epsilon, \sigma, \sigma^{\prime}, \delta, \Omega\right)$ with $\|U(\tau, z)\|_{\left(\epsilon, \sigma, \sigma^{\prime}, \delta, \Omega\right)} \leq R$. Under the assumptions (74), from Proposition 4 and Corollary 1, we get a constant $C_{5}>0$ (independent of $\epsilon$ ) such that

$$
\begin{array}{rl}
\| a_{n, \underline{k}}(\tau, z, \epsilon) \epsilon^{-n} \int_{0}^{\tau}(\tau-h)^{s} \exp \left(-k_{2} h\right) h^{k_{0}} \partial_{z}^{k_{1}-S} & U(h, z) d h \|_{\left(\epsilon, \sigma, \sigma^{\prime}, \delta, \Omega\right)} \\
& \leq C_{5}|\epsilon|^{s+k_{0}+1-n} \delta^{S-k_{1}}\|U(\tau, z)\|_{\left(\epsilon, \sigma, \sigma^{\prime}, \delta, \Omega\right)}
\end{array}
$$

for all $\underline{k} \in \mathcal{A}_{1}$, all $n \in I_{k}$.

Again under the assumptions (74) with the help of Propositions 3,1) and Proposition 5 and the estimates (71), (84), we get constants $C_{6}, C_{7}>0$ (independent of $\epsilon$ ) such that

$$
\begin{aligned}
& \left\|a_{n, \underline{k}}(\tau, z, \epsilon) \epsilon^{-n} \int_{0}^{\tau}(\tau-h)^{s} \exp \left(-k_{2} h\right) h^{k_{0}} \partial_{z}^{k_{1}} w(h, z, \epsilon)\right\|_{\left(\epsilon, \sigma, \sigma^{\prime}, \delta, \Omega\right)} \\
& \leq C_{6}|\epsilon|^{1-n}\left\|\tau^{s}\right\|_{\left(\epsilon, \sigma, \sigma^{\prime}, \delta, \Omega\right)}|| \exp \left(-k_{2} \tau\right) \tau^{k_{0}} \partial_{z}^{k_{1}} w(\tau, z, \epsilon) \|_{\left(\epsilon, \sigma, \sigma^{\prime}, \delta, \Omega\right)} \\
& \quad \leq C_{7}|\epsilon|^{s+k_{0}+1-n}\left\|\partial_{z}^{k_{1}} w(\tau, z, \epsilon)\right\|_{\left(\epsilon, \tilde{\sigma}, \tilde{\sigma}^{\prime}, \delta, \Omega\right)} \leq C_{7} I|\epsilon|^{s+k_{0}+1-n}
\end{aligned}
$$

for all $\underline{k} \in \mathcal{A}_{1}$, all $n \in I_{\underline{k}}$.

On the other hand, since the convolution product is commutative, from the binomal formula, we can write

$$
\begin{aligned}
&\left(\partial_{z}^{-S} U(\tau, z)+w(\tau, z, \epsilon)\right)^{* l}=\left(\partial_{z}^{-S} U(\tau, z)\right)^{* l}+(w(\tau, z, \epsilon))^{* l} \\
&+\sum_{l^{1}+l^{2}=l, l^{1} \geq 1, l^{2} \geq 1} \frac{l !}{l^{1} ! l^{2} !}\left(\partial_{z}^{-S} U(\tau, z)\right)^{* l^{1}} *(w(\tau, z, \epsilon))^{* l^{2}}
\end{aligned}
$$

for all $l \geq 2$. From Proposition 2, 2) and Proposition 5 we get a constant $C_{8}>0$ (independent of $\epsilon$ ) such that

$$
\begin{aligned}
& \left\|\left(\partial_{z}^{-S} U(\tau, z)+w(\tau, z, \epsilon)\right)^{* l}\right\|_{\left(\epsilon, \sigma, \sigma^{\prime}, \delta, \Omega\right)} \\
& \quad \leq C_{8}|\epsilon|^{l-1}\left(\delta^{S l} R^{l}+I^{l}+\sum_{l^{1}+l^{2}=l, l^{1} \geq 1, l^{2} \geq 1} \frac{l !}{l^{1} ! l^{2} !} \delta^{S l^{1}} R^{l^{1}} I^{l^{2}}\right)=C_{8}|\epsilon|^{l-1}\left(\delta^{S} R+I\right)^{l}
\end{aligned}
$$

for all $l \in \mathcal{A}_{2}$. From Proposition 4 we get a constant $C_{9}>0$ (independent of $\epsilon$ ) such that

$$
\begin{array}{r}
\left.\| \alpha_{l}(\tau, z, \epsilon)\left(\partial_{z}^{-S} U(\tau, z)+w(\tau, z, \epsilon)\right)^{* l}\right) \|_{\left(\epsilon, \sigma, \sigma^{\prime}, \delta, \Omega\right)} \\
\quad \leq C_{9}\left\|\left(\partial_{z}^{-S} U(\tau, z)+w(\tau, z, \epsilon)\right)^{* l}\right\|_{\left(\epsilon, \sigma, \sigma^{\prime}, \delta, \Omega\right)}
\end{array}
$$

for all $l \in \mathcal{A}_{2}$. From (87) and (88), we get that

$$
\left.\| \alpha_{l}(\tau, z, \epsilon)\left(\partial_{z}^{-S} U(\tau, z)+w(\tau, z, \epsilon)\right)^{* l}\right) \|_{\left(\epsilon, \sigma, \sigma^{\prime}, \delta, \Omega\right)} \leq C_{8} C_{9}|\epsilon|^{l-1}\left(\delta^{S} R+I\right)^{l}
$$

for all $l \in \mathcal{A}_{2}$. Now, we choose $\delta, R, I>0$ such that

$$
\sum_{\underline{k} \in \mathcal{A}_{1}} \sum_{n \in I_{\underline{k}}}|\epsilon|^{s+k_{0}+1-n}\left(C_{5} \delta^{S-k_{1}} R+C_{7} I\right)+\sum_{l \in \mathcal{A}_{2}} C_{8} C_{9}|\epsilon|^{l-1}\left(\delta^{S} R+I\right)^{l} \leq R
$$

for all $\epsilon \in \mathcal{E}$. From the inequalities (85), (86) and (89), we deduce that

$$
\left\|\mathcal{A}_{\epsilon}(U(\tau, z))\right\|_{\left(\epsilon, \sigma, \sigma^{\prime}, \delta, \Omega\right)} \leq R
$$


for all $\epsilon \in \mathcal{E}$.

We prove now the estimates (83).

Let $R>0$ and let $U_{1}, U_{2} \in B(0, R)$. Under the assumptions (74), from Proposition 4 and Corollary 1,1 ) we get a constant $C_{10}>0$ (independent of $\epsilon$ ) with

$$
\begin{array}{r}
\left\|a_{n, \underline{k}}(\tau, z, \epsilon) \epsilon^{-n} \int_{0}^{\tau}(\tau-h)^{s} \exp \left(-k_{2} h\right) h^{k_{0}} \partial_{z}^{k_{1}-S}\left(U_{1}(h, z)-U_{2}(h, z)\right) d h\right\|_{\left(\epsilon, \sigma, \sigma^{\prime}, \delta, \Omega\right)} \\
\leq C_{10}|\epsilon|^{s+k_{0}+1-n} \delta^{S-k_{1}}\left\|U_{1}(\tau, z)-U_{2}(\tau, z)\right\|_{\left(\epsilon, \sigma, \sigma^{\prime}, \delta, \Omega\right)}
\end{array}
$$

for all $\underline{k} \in \mathcal{A}_{1}$, all $n \in I_{\underline{k}}$. As in the part a), we can write from the binomial formula

$$
\begin{aligned}
&\left(\partial_{z}^{-S} U_{1}(\tau, z)+w(\tau, z, \epsilon)\right)^{* l}-\left(\partial_{z}^{-S} U_{2}(\tau, z)+w(\tau, z, \epsilon)\right)^{* l}=\left(\partial_{z}^{-S} U_{1}(\tau, z)\right)^{* l}-\left(\partial_{z}^{-S} U_{2}(\tau, z)\right)^{* l} \\
&+\sum_{l^{1}+l^{2}=l, l^{1} \geq 1, l^{2} \geq 1} \frac{l !}{l^{1} ! l^{2 !}}\left(\left(\partial_{z}^{-S} U_{1}(\tau, z)\right)^{* l^{1}}-\left(\partial_{z}^{-S} U_{2}(\tau, z)\right)^{* l^{1}}\right) *(w(\tau, z, \epsilon))^{* l^{2}}
\end{aligned}
$$

for all $l \geq 2$. On the other hand, we have that

$$
\begin{aligned}
& \left(\partial_{z}^{-S} U_{1}(\tau, z)\right)^{* 2}-\left(\partial_{z}^{-S} U_{2}(\tau, z)\right)^{* 2} \\
& \quad=\left(\partial_{z}^{-S} U_{1}(\tau, z)-\partial_{z}^{-S} U_{2}(\tau, z)\right) *\left(\partial_{z}^{-S} U_{1}(\tau, z)+\partial_{z}^{-S} U_{2}(\tau, z)\right)
\end{aligned}
$$

and, for all $l^{1} \geq 3$, we can write

$$
\begin{aligned}
\left(\partial_{z}^{-S} U_{1}(\tau, z)\right)^{* l^{1}}-\left(\partial_{z}^{-S} U_{2}(\tau, z)\right)^{* l^{1}}=\left(\partial_{z}^{-S} U_{1}(\tau, z)-\partial_{z}^{-S} U_{2}(\tau, z)\right) & \\
*\left(\left(\partial_{z}^{-S} U_{1}(\tau, z)\right)^{* l^{1}-1}+\left(\partial_{z}^{-S} U_{2}(\tau, z)\right)^{* l^{1}-1}\right. & \\
& \left.+\sum_{k=1}^{l^{1}-2}\left(\partial_{z}^{-S} U_{2}(\tau, z)\right)^{* k} *\left(\partial_{z}^{-S} U_{1}(\tau, z)\right)^{* l^{1}-k-1}\right) .
\end{aligned}
$$

Using (93) and (94), from Propositions 2, 2) and 5 we get a constant $C_{11}>0$ (independent of $\epsilon)$ such that

$$
\begin{aligned}
\|\left(\partial_{z}^{-S} U_{1}(\tau, z)\right)^{* l^{1}}-\left(\partial_{z}^{-S} U_{2}(\tau, z)\right)^{* l^{1}} & \|_{\left(\epsilon, \sigma, \sigma^{\prime}, \delta, \Omega\right)} \\
& \leq\left(C_{11}|\epsilon|^{l^{1}-1} \delta^{S l^{1}} R^{l^{1}-1}\right)\left\|U_{1}(\tau, z)-U_{2}(\tau, z)\right\|_{\left(\epsilon, \sigma, \sigma^{\prime}, \delta, \Omega\right)}
\end{aligned}
$$

for all $l^{1} \in \mathcal{A}_{2}$. From (92), (95), we get a constant $C_{12}>0$ (independent of $\epsilon$ ) such that

$$
\begin{aligned}
& \left\|\left(\partial_{z}^{-S} U_{1}(\tau, z)+w(\tau, z, \epsilon)\right)^{* l}-\left(\partial_{z}^{-S} U_{2}(\tau, z)+w(\tau, z, \epsilon)\right)^{* l}\right\|_{\left(\epsilon, \sigma, \sigma^{\prime}, \delta, \Omega\right)} \\
& \leq C_{12}|\epsilon|^{l-1}\left(\delta^{S l} R^{l-1}+\sum_{\begin{array}{l}
l^{1}+l^{2}=l, l^{1} \geq 1, l^{2} \geq 1 \\
\end{array}} \frac{l !}{l^{1} ! l^{2} !} \delta^{S l^{1}} R^{l^{1}-1} I^{l^{2}}\right)\left\|U_{1}(\tau, z)-U_{2}(\tau, z)\right\|_{\left(\epsilon, \sigma, \sigma^{\prime}, \delta, \Omega\right)} \\
& \quad=C_{12}|\epsilon|^{l-1} R^{-1}\left(\left(\delta^{S} R+I\right)^{l}-I^{l}\right)\left\|U_{1}(\tau, z)-U_{2}(\tau, z)\right\|_{\left(\epsilon, \sigma, \sigma^{\prime}, \delta, \Omega\right)}
\end{aligned}
$$

for all $l \in \mathcal{A}_{2}$. From Proposition 4 we get a constant $C_{13}>0$ (independent of $\epsilon$ ) such that

$$
\begin{aligned}
& \left\|\alpha_{l}(\tau, z, \epsilon)\left(\left(\partial_{z}^{-S} U_{1}(\tau, z)+w(\tau, z, \epsilon)\right)^{* l}-\left(\partial_{z}^{-S} U_{2}(\tau, z)+w(\tau, z, \epsilon)\right)^{* l}\right)\right\|_{\left(\epsilon, \sigma, \sigma^{\prime}, \delta, \Omega\right)} \\
& \quad \leq C_{13}\left\|\left(\partial_{z}^{-S} U_{1}(\tau, z)+w(\tau, z, \epsilon)\right)^{* l}-\left(\partial_{z}^{-S} U_{2}(\tau, z)+w(\tau, z, \epsilon)\right)^{* l}\right\|_{\left(\epsilon, \sigma, \sigma^{\prime}, \delta, \Omega\right)}
\end{aligned}
$$


for all $l \in \mathcal{A}_{2}$. Gathering (96) and (97), we get that

$$
\begin{aligned}
\left\|\alpha_{l}(\tau, z, \epsilon)\left(\left(\partial_{z}^{-S} U_{1}(\tau, z)+w(\tau, z, \epsilon)\right)^{* l}-\left(\partial_{z}^{-S} U_{2}(\tau, z)+w(\tau, z, \epsilon)\right)^{* l}\right)\right\|_{\left(\epsilon, \sigma, \sigma^{\prime}, \delta, \Omega\right)} \\
\leq C_{12} C_{13}|\epsilon|^{l-1} R^{-1}\left(\left(\delta^{S} R+I\right)^{l}-I^{l}\right)\left\|U_{1}(\tau, z)-U_{2}(\tau, z)\right\|_{\left(\epsilon, \sigma, \sigma^{\prime}, \delta, \Omega\right)}
\end{aligned}
$$

for all $l \in \mathcal{A}_{2}$. Now, we choose $\delta, R, I>0$ such that

$$
\sum_{\underline{k} \in \mathcal{A}_{1}} \sum_{n \in I_{\underline{k}}} C_{10}|\epsilon|^{s+k_{0}+1-n} \delta^{S-k_{1}}+\sum_{l \in \mathcal{A}_{2}} C_{12} C_{13}|\epsilon|^{l-1} R^{-1}\left(\left(\delta^{S} R+I\right)^{l}-I^{l}\right) \leq \frac{1}{2}
$$

for all $\epsilon \in \mathcal{E}$. From the inequalities (91), (98), we deduce that

$$
\left\|A_{\epsilon}\left(U_{1}(\tau, z)\right)-A_{\epsilon}\left(U_{2}(\tau, z)\right)\right\|_{\left(\epsilon, \sigma, \sigma^{\prime}, \delta, \Omega\right)} \leq \frac{1}{2}\left\|U_{1}(\tau, z)-U_{2}(\tau, z)\right\|_{\left(\epsilon, \sigma, \sigma^{\prime}, \delta, \Omega\right)},
$$

for all $\epsilon \in \mathcal{E}$.

Finally, we choose $\delta, R, I>0$ is such a way that the conditions (90) and (99) hold simultaneously. This yields Lemma 8.

Now, let the assumptions (74), (75) hold. We choose the constants $I, R, \delta$ as in the lemma 8 . Assume that

$$
\sum_{j=0}^{S-1-h}\left\|V_{j+h}(\tau, \epsilon)\right\|_{0, \epsilon, \tilde{\sigma}, \tilde{\sigma}^{\prime}, \Omega} \frac{\delta^{j}}{j !} \leq I,
$$

for all $0 \leq h \leq S-1$, for all $\epsilon \in \mathcal{E}$. From Lemma 8 and the classical contractive mapping theorem on complete metric spaces, we deduce that the map $A_{\epsilon}$ has a unique fixed point (called $U(\tau, z, \epsilon)$ ) in the closed ball $B(0, R) \subset S G\left(\epsilon, \sigma, \sigma^{\prime}, \delta, \Omega\right)$, for all $\epsilon$ in $\mathcal{E}$, which means that $A_{\epsilon}(U(\tau, z, \epsilon))=$ $U(\tau, z, \epsilon)$ with $\|U\|_{\left(\epsilon, \sigma, \sigma^{\prime}, \delta, \Omega\right)} \leq R$. Finally, we get that the function

$$
V(\tau, z, \epsilon)=\partial_{z}^{-S} U(\tau, z, \epsilon)+w(\tau, z, \epsilon)
$$

satisfies the Cauchy problem (73), (77), for all $\tau \in S_{d} \cup D(0, r)$, all $z \in D(0, \delta)$, all $\epsilon \in \mathcal{E}$. Moreover, from Proposition 2, 2) we deduce that

$$
\|V(\tau, z, \epsilon)\|_{\left(\epsilon, \sigma, \sigma^{\prime}, \delta, \Omega\right)} \leq \delta^{S} R+I
$$

for all $\epsilon \in \mathcal{E}$.

\section{Analytic solutions in a complex parameter of a singularly per- turbed Cauchy problem}

Definition 2 Let $V(\tau, \epsilon)$ be a holomorphic function on some punctured polydisc

$$
\Omega_{\tau_{0}, \epsilon_{0}}=D\left(0, \tau_{0}\right) \times\left(D\left(0, \epsilon_{0}\right) \backslash\{0\}\right)
$$

where $0<\tau_{0}<|a|$ and $0<\epsilon_{0}<1$, with $a \in \mathbb{C}^{*}$ such that $\arg (a) \neq 0$. We make the assumption

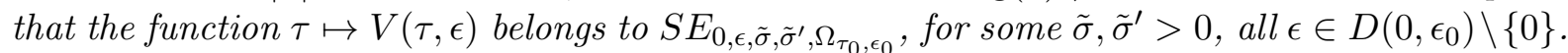

Let $U_{d}$ be an open unbounded sector centered at 0 , with bisecting direction d and with small opening. Let $\mathcal{E}$ be an open sector centered at 0 such that $\mathcal{E} \subset D\left(0, \epsilon_{0}\right)$. We denote by

$$
\Omega(d, \mathcal{E})=\left(U_{d} \cup D\left(0, \tau_{0}\right)\right) \times \mathcal{E} .
$$


We also assume that there exists an open unbounded sector $\mathcal{T}$ centered at 0 such that

$$
d+\arg (t)-\arg (\epsilon) \in(-\pi / 2, \pi / 2)
$$

for all $t \in \mathcal{T}$.

1) Let $d \in(-\pi / 2, \pi / 2)$ with $d \neq \arg (a)$. We assume that $a \notin U_{d}$. We assume that the function $(\tau, \epsilon) \mapsto V(\tau, \epsilon)$ can be extended to an analytic function $(\tau, \epsilon) \mapsto V_{U_{d}, \mathcal{E}}(\tau, \epsilon)$ on $\Omega(d, \mathcal{E})$ and that the function $\tau \mapsto V_{U_{d}, \mathcal{E}}(\tau, \epsilon)$ belongs to $S E_{0, \epsilon, \tilde{\sigma}, 0, \Omega(d, \mathcal{E})}$, for all $\epsilon \in \mathcal{E}$.

2) Let $d \in[-\pi,-\pi / 2) \cup(\pi / 2, \pi]$ with $d \neq \arg (a)$. We assume that $a \notin U_{d}$. We assume that the function $(\tau, \epsilon) \mapsto V(\tau, \epsilon)$ can be extended to an analytic function $(\tau, \epsilon) \mapsto V_{U_{d}, \mathcal{E}}(\tau, \epsilon)$ on $\Omega(d, \mathcal{E})$ and that the function $\tau \mapsto V_{U_{d}, \mathcal{E}}(\tau, \epsilon)$ belongs to $S E_{0, \epsilon, \tilde{\sigma}, \tilde{\sigma}^{\prime}, \Omega(d, \mathcal{E})}$, for all $\epsilon \in \mathcal{E}$.

If 1) holds, we say that the set $\left\{V, V_{U_{d}, \mathcal{E}}, a, \tilde{\sigma}, \tilde{\sigma}^{\prime}, \mathcal{T}\right\}$ is ( $\left.\tilde{\sigma}, 0\right)$-admissible and if 2) holds, we say that the set $\left\{V, V_{U_{d}}, \mathcal{E}, a, \tilde{\sigma}, \tilde{\sigma}^{\prime}, \mathcal{T}\right\}$ is $\left(\tilde{\sigma}, \tilde{\sigma}^{\prime}\right)$-admissible.

Let $\mathcal{A}_{1}\left(\right.$ resp. $\left.\mathcal{A}_{2}\right)$ be a finite subset of $\mathbb{N}^{4}$ (resp. $\mathbb{N}$ ). For all $\underline{k}=\left(s, k_{0}, k_{1}, k_{2}\right) \in \mathcal{A}_{1}$, we denote by $b_{\underline{k}}(z, \epsilon)$ some bounded holomorphic function on $D(0, \rho) \times \mathcal{E}$, for some $\rho>0$. For all $l \in \mathcal{A}_{2}$, we denote by $c_{l}(z, \epsilon)$ some bounded holomorphic function on $D(0, \rho) \times \mathcal{E}$. Let $a \in \mathbb{C}^{*}$ be a complex number with $\arg (a) \neq 0, S \geq 1$ be an integer and $d \in(-\pi / 2, \pi / 2)$ with $d \neq \arg (a)$. We consider the following singularly perturbed Cauchy problem

$$
\begin{aligned}
\epsilon \partial_{t} \partial_{z}^{S} X_{U_{d}, \mathcal{E}}(t, z, \epsilon)+a \partial_{z}^{S} X_{U_{d}, \mathcal{E}}(t, z, \epsilon) & \\
\quad & =\sum_{\underline{k}=\left(s, k_{0}, k_{1}, k_{2}\right) \in \mathcal{A}_{1}} \frac{\underline{b_{k}}(z, \epsilon)}{t^{s+1}}\left(\partial_{t}^{k_{0}} \partial_{z}^{k_{1}} X_{U_{d}, \mathcal{E}}\right)\left(t+k_{2} \epsilon, z, \epsilon\right)+\sum_{l \in \mathcal{A}_{2}} c_{l}(z, \epsilon) X_{U_{d}, \mathcal{E}}^{l}(t, z, \epsilon)
\end{aligned}
$$

for given initial data

$$
\left(\partial_{z}^{j} X_{U_{d}, \mathcal{E}}\right)(t, 0, \epsilon)=\varphi_{j, U_{d}, \mathcal{E}}(t, \epsilon) \quad, \quad 0 \leq j \leq S-1,
$$

where the functions $\varphi_{j, U_{d}, \mathcal{E}}(t, \epsilon)$ are constructed in the following manner. For all $0 \leq j \leq S-1$, let $\left\{V_{j}, V_{j, U_{d}, \mathcal{E}}, a, \tilde{\sigma}, \tilde{\sigma}^{\prime}, \mathcal{T}\right\}$ be a $(\tilde{\sigma}, 0)$-admissible set, then we consider the function

$$
\varphi_{j, U_{d}, \mathcal{E}}(t, \epsilon)=\int_{L_{\gamma}} V_{j, U_{d}, \mathcal{E}}(\tau, \epsilon) \exp \left(-\frac{t \tau}{\epsilon}\right) d \tau
$$

where $L_{\gamma}=\mathbb{R}_{+} e^{i \gamma} \subset U_{d} \cup\{0\}$ is a halfline where $\gamma$ depends on $t$ and $\epsilon$ in such a way that there exists $\delta_{1}>0$ with

$$
\cos (\gamma+\arg (t)-\arg (\epsilon)) \geq \delta_{1}
$$

for all $t \in \mathcal{T}$ with $|t|>\tilde{\sigma} / \delta_{1}$ and $\epsilon \in \mathcal{E}$. By construction, the function $\varphi_{j, U_{d}, \mathcal{E}}(t, \epsilon)$ is holomorphic and bounded on $\left(\mathcal{T} \cap\left\{|t|>\tilde{\sigma} / \delta_{1}\right\}\right) \times \mathcal{E}$.

Proposition 7 We make the following assumptions.

There exist real numbers $\sigma^{\prime}>\tilde{\sigma}^{\prime}>0$ such that, for all $\underline{k} \in \mathcal{A}_{1}$, we have

$$
S \geq k_{1}+b k_{0}+\frac{k_{2} b}{\sigma^{\prime}} \quad, \quad S>k_{1},
$$

and for all $l \in \mathcal{A}_{2}$, we have

$$
l \geq 2 .
$$


Then, there exist constants $I>0$ and $\delta>0$ (independent of $\epsilon$ ) such that if we assume that

$$
\sum_{j=0}^{S-1-h}\left\|V_{j+h}(\tau, \epsilon)\right\|_{0, \epsilon, \tilde{\sigma}, 0, \Omega_{\tau_{0}, \epsilon_{0}}} \frac{\delta^{j}}{j !} \leq I \quad, \quad \sum_{j=0}^{S-1-h}\left\|V_{j+h, U_{d}, \mathcal{E}}(\tau, \epsilon)\right\|_{0, \epsilon, \tilde{\sigma}, 0, \Omega(d, \mathcal{E})} \frac{\delta^{j}}{j !} \leq I,
$$

for all $0 \leq h \leq S-1$, for all $\epsilon \in \mathcal{E}$, the Cauchy problem (100), (101) has a solution $(t, z, \epsilon) \mapsto$ $X_{U_{d}, \mathcal{E}}(t, z, \epsilon)$ which is holomorphic and bounded on a domain $(t, z, \epsilon) \in\left(\mathcal{T} \cap\left\{|t|>\sigma \zeta(b) / \delta_{1}\right\}\right) \times$ $D(0, \delta / 2) \times \mathcal{E}$ for some $\sigma>\tilde{\sigma}$. The function $X_{U_{d}, \mathcal{E}}(t, z, \epsilon)$ can be written as a Laplace transform

$$
X_{U_{d}, \mathcal{E}}(t, z, \epsilon)=\int_{L_{\gamma}} V_{U_{d}, \mathcal{E}}(\tau, z, \epsilon) \exp \left(-\frac{t \tau}{\epsilon}\right) d \tau
$$

where $V_{U_{d}, \mathcal{E}}(\tau, z, \epsilon)$ is a holomorphic function on the domain $\left(U_{d} \cup D\left(0, \tau_{0}\right)\right) \times D(0, \delta / 2) \times \mathcal{E}$ and satisfies the following estimates: there exists a constant $C_{\Omega(d, \mathcal{E})}>0$ (independent of $\epsilon$ ) such that

$$
\left|V_{U_{d}, \mathcal{E}}(\tau, z, \epsilon)\right| \leq C_{\Omega(d, \mathcal{E})}\left(1+\frac{|\tau|^{2}}{|\epsilon|^{2}}\right)^{-1} \exp \left(\frac{\sigma \zeta(b)}{|\epsilon|}|\tau|\right)
$$

for all $(\tau, z, \epsilon) \in\left(U_{d} \cup D\left(0, \tau_{0}\right)\right) \times D(0, \delta / 2) \times \mathcal{E}$. Moreover, the function $V_{U_{d}, \mathcal{E}}(\tau, z, \epsilon)$ is the analytic continuation of a function $V(\tau, z, \epsilon)$ which is holomorphic on a punctured polydisc $D\left(0, \tau_{0}\right) \times D(0, \delta / 2) \times\left(D\left(0, \epsilon_{0}\right) \backslash\{0\}\right)$ and fulfills the next estimates: there exists a constant $C_{\Omega_{\tau_{0}, \epsilon_{0}}}>0$ (independent of $\epsilon$ ) such that

$$
|V(\tau, z, \epsilon)| \leq C_{\Omega_{\tau_{0}, \epsilon_{0}}}\left(1+\frac{|\tau|^{2}}{|\epsilon|^{2}}\right)^{-1} \exp \left(\frac{\sigma \zeta(b)}{|\epsilon|}|\tau|\right)
$$

for all $\tau \in D\left(0, \tau_{0}\right)$, all $z \in D(0, \delta / 2)$ and all $\epsilon \in D\left(0, \epsilon_{0}\right) \backslash\{0\}$.

Proof We consider the following Cauchy problem

$$
\begin{aligned}
& \partial_{z}^{S} V(\tau, z, \epsilon) \\
& \quad=\sum_{\underline{k}=\left(s, k_{0}, k_{1}, k_{2}\right) \in \mathcal{A}_{1}} \frac{(-1)^{k_{0}} b_{\underline{k}}(z, \epsilon)}{(a-\tau) \epsilon^{1+s+k_{0}} s !} \int_{0}^{\tau}(\tau-h)^{s} \exp \left(-k_{2} h\right) h^{k_{0}} \partial_{z}^{k_{1}} V(h, z, \epsilon) d h \\
& +\sum_{l \in \mathcal{A}_{2}} \frac{c_{l}(z, \epsilon)}{a-\tau} V^{* l}(\tau, z, \epsilon)
\end{aligned}
$$

for the given initial data

$$
\left(\partial_{z}^{j} V\right)(\tau, 0, \epsilon)=V_{j}(\tau, \epsilon) \quad, \quad 0 \leq j \leq S-1 .
$$

From the assumptions (102), (103) together with (104) we deduce that the conditions (78), (79) and (80) from Proposition 6, 2) are fulfilled for the problem (108), (109). We deduce that the problem (108), (109) has a unique solution $V(\tau, z, \epsilon)$ that belongs to the space $S G\left(\epsilon, \sigma, 0, \delta, \Omega_{\tau_{0}, \epsilon_{0}}\right)$, for some $\sigma>\tilde{\sigma}$. In particular, $V(\tau, z, \epsilon)$ is holomorphic on the punctured polydisc $D\left(0, \tau_{0}\right) \times$ $D(0, \delta / 2) \times\left(D\left(0, \epsilon_{0}\right) \backslash\{0\}\right)$ and using Proposition 1, it satisfies also (107).

In the second step of the proof, we show that the function $V(\tau, z, \epsilon)$ can be analytically continued to a function $V_{U_{d}, \mathcal{E}}(\tau, z, \epsilon)$ on $\left(U_{d} \cup D\left(0, \tau_{0}\right)\right) \times D(0, \delta / 2) \times \mathcal{E}$ which satisfies (106). 
Indeed, by construction, the function $V(\tau, z, \epsilon)$ solves also the problem

$$
\begin{aligned}
& \partial_{z}^{S} V(\tau, z, \epsilon) \\
& \quad=\sum_{\underline{k}=\left(s, k_{0}, k_{1}, k_{2}\right) \in \mathcal{A}_{1}} \frac{(-1)^{k_{0}} b_{\underline{k}}(z, \epsilon)}{(a-\tau) \epsilon^{1+s+k_{0}} s !} \int_{0}^{\tau}(\tau-h)^{s} \exp \left(-k_{2} h\right) h^{k_{0}} \partial_{z}^{k_{1}} V(h, z, \epsilon) d h \\
& +\sum_{l \in \mathcal{A}_{2}} \frac{c_{l}(z, \epsilon)}{a-\tau} V^{* l}(\tau, z, \epsilon)
\end{aligned}
$$

with the given initial conditions

$$
\left(\partial_{z}^{j} V\right)(\tau, 0, \epsilon)=V_{j, U_{d}, \mathcal{E}}(\tau, \epsilon) \quad, \quad 0 \leq j \leq S-1,
$$

for all $\tau \in D\left(0, \tau_{0}\right), z \in D(0, \delta / 2)$ and $\epsilon \in \mathcal{E}$. Gathering the assumptions (102), (103) and (104) we deduce that the conditions (78), (79) and (80) from Proposition 6,2) are fulfilled for the problem (110), (111). We get that the problem (110), (111) has a unique solution $V_{U_{d}, \mathcal{E}}(\tau, z, \epsilon)$ that belongs to the space $S G(\epsilon, \sigma, 0, \delta, \Omega(d, \mathcal{E}))$ for some $\sigma>\tilde{\sigma}$. In particular, $V_{U_{d}, \mathcal{E}}(\tau, z, \epsilon)$ defines a holomorphic function on $\left(U_{d} \cup D\left(0, \tau_{0}\right)\right) \times D(0, \delta / 2) \times \mathcal{E}$, coincides with $V(\tau, z, \epsilon)$ on $D\left(0, \tau_{0}\right) \times D(0, \delta / 2) \times \mathcal{E}$ and fills (106) due to Proposition 1.

In the last part of the proof, it remains to show that the Laplace transform

$$
X_{U_{d}, \mathcal{E}}(t, z, \epsilon)=\int_{L_{\gamma}} V_{U_{d}, \mathcal{E}}(\tau, z, \epsilon) \exp \left(-\frac{t \tau}{\epsilon}\right) d \tau
$$

satisfies the problem $(100),(101)$ on the domain $\left(\mathcal{T} \cap\left\{|t|>\sigma \zeta(b) / \delta_{1}\right\}\right) \times D(0, \delta / 2) \times \mathcal{E}$. This is a consequence of the classical properties of the Laplace transform that we recall in the next lemma, see [2], [9] for references.

Lemma 9 Let $m \geq 0$ be an integer. Let $w_{1}(\tau), w_{2}(\tau)$ be holomorphic functions on the unbounded sector $U_{d}$ such that there exist $C, K>0$ with

$$
\left|w_{j}(\tau)\right| \leq C \exp (K|\tau|) \quad, \quad j=1,2
$$

for all $\tau \in U_{d}$. We denote

$$
w_{1} * w_{2}(\tau)=\int_{0}^{\tau} w_{1}(\tau-s) w_{2}(s) d s
$$

their convolution product on $U_{d}$. We denote by $\mathcal{D}$ an unbounded sector centered at 0 for which there exists $\delta_{1}>0$ with

$$
d+\arg (t) \in(-\pi / 2, \pi / 2) \quad, \quad \cos (d+\arg (t)) \geq \delta_{1},
$$

for all $t \in \mathcal{D}$. Then the following identities hold for the Laplace transforms

$$
\begin{array}{r}
\int_{L_{d}} \tau^{m} \exp (-t \tau) d \tau=\frac{m !}{t^{m+1}} \quad, \quad \partial_{t}\left(\int_{L_{d}} w_{1}(\tau) \exp (-t \tau) d \tau\right)=\int_{L_{d}}(-\tau) w_{1}(\tau) \exp (-t \tau) d \tau \\
\int_{L_{d}} w_{1} * w_{2}(\tau) \exp (-t \tau) d \tau=\left(\int_{L_{d}} w_{1}(\tau) \exp (-t \tau) d \tau\right)\left(\int_{L_{d}} w_{2}(\tau) \exp (-t \tau) d \tau\right)
\end{array}
$$

where $L_{d}=\mathbb{R}_{+} e^{i d} \subset U_{d} \cup\{0\}$, for all $t \in \mathcal{D} \cap\left\{|t|>K / \delta_{1}\right\}$. 


\section{Construction of a Banach valued cocyle}

We keep the notations of Section 3. We recall the definition of a good covering.

Definition 3 For all $0 \leq i \leq \nu-1$, we consider open sectors $\mathcal{E}_{i}$ centered at 0 , with radius $\epsilon_{0}$ such that $\mathcal{E}_{i} \cap \mathcal{E}_{i+1} \neq \emptyset$, for all $0 \leq i \leq \nu-1$ (with the convention that $\mathcal{E}_{\nu}=\mathcal{E}_{0}$ ), which are three by three disjoint and such that $\cup_{i=0}^{\nu-1} \mathcal{E}_{i}=\mathcal{U} \backslash\{0\}$, where $\mathcal{U}$ is some neighborhood of 0 in $\mathbb{C}$. Such a set of sectors $\left\{\mathcal{E}_{i}\right\}_{0 \leq i \leq \nu-1}$ is called a good covering in $\mathbb{C}^{*}$.

Let $\left\{\mathcal{E}_{i}\right\}_{0 \leq i \leq \nu-1}$ be a good covering in $\mathbb{C}^{*}$. For all $0 \leq j \leq S-1$, for all $0 \leq i \leq \nu-1$, we consider directions $d_{i} \in \mathbb{R}$ with $d_{i} \neq \arg (a), 0 \leq i \leq \nu-1$, for some $a \in \mathbb{C}^{*}$ and a family of sets $\left\{V_{j}, V_{j, U_{d_{i}}, \mathcal{E}_{i}}, a, \tilde{\sigma}, \tilde{\sigma}^{\prime}, \mathcal{T}\right\}$ which are $(\tilde{\sigma}, 0)$-admissible when $d_{i} \in(-\pi / 2, \pi / 2)$ and $\left(\tilde{\sigma}, \tilde{\sigma}^{\prime}\right)$-admissible when $d_{i} \in[-\pi,-\pi / 2) \cup(\pi / 2, \pi]$. We make the assumption that there exists a least one integer $0 \leq i_{0} \leq \nu-1$ such that $d_{i_{0}}, d_{i_{0}+1} \in[-\pi,-\pi / 2) \cup(\pi / 2, \pi]$ with $U_{d_{i_{0}}} \cap U_{d_{i_{0}+1}} \neq \emptyset$.

For $d_{i} \in(-\pi / 2, \pi / 2)$, we consider the Laplace transform

$$
\varphi_{j, U_{d_{i}}, \mathcal{E}_{i}}(t, \epsilon)=\int_{L_{\gamma_{i}}} V_{j, U_{d_{i}}, \mathcal{E}_{i}}(\tau, \epsilon) \exp \left(-\frac{t \tau}{\epsilon}\right) d \tau
$$

where $L_{\gamma_{i}}=\mathbb{R}_{+} e^{\sqrt{-1} \gamma_{i}} \subset U_{d_{i}} \cup\{0\}$ is a halfline where $\gamma_{i}$ may depend on $t$ and $\epsilon$ in such a way that there exists $\delta_{1}>0$ with

$$
\cos \left(\gamma_{i}+\arg (t)-\arg (\epsilon)\right) \geq \delta_{1}
$$

for all $t \in \mathcal{T}$ with $|t|>\tilde{\sigma} / \delta_{1}$ and $\epsilon \in \mathcal{E}_{i}$.

We choose a real number $\sigma^{\prime \prime}>\tilde{\sigma}^{\prime}$ and a real number $\theta_{i}$ such that

$$
\arg (t)-\arg (\epsilon)+\theta_{i} \neq \pi
$$

for all $t \in \mathcal{T}$, all $\epsilon \in \mathcal{E}_{i}$. The function $\log (z)$ denotes also the principal branch of the logarithm of $z$ which is holomorphic on $\left\{z \in \mathbb{C}^{*} / \arg (z) \neq \pi\right\}$.

For $d_{i} \in[-\pi,-\pi / 2) \cup(\pi / 2, \pi]$, we choose $\gamma_{i}$ with $e^{\sqrt{-1} \gamma_{i}} \in U_{d_{i}}$ that may depend on $t$ and $\epsilon$ in such a way that there exists $\delta_{1}>0$ with

$$
\cos \left(\gamma_{i}+\arg (t)-\arg (\epsilon)\right) \geq \delta_{1}
$$

for all $t \in \mathcal{T}$, all $\epsilon \in \mathcal{E}_{i}$. Due to the formula

$$
\arg \left(\log \left(\frac{t}{\epsilon} e^{\sqrt{-1} \theta_{i}}\right)\right)=\arctan \left(\frac{\arg (t)-\arg (\epsilon)+\theta_{i}}{\log \left|\frac{t}{\epsilon}\right|}\right)
$$

for all $t \in \mathcal{T}$ with $|t|>\mu_{\mathcal{T}}$ provided that $\mu_{\mathcal{T}}>0$ is large enough, all $\epsilon \in \mathcal{E}_{i}$, we notice that $e^{\sqrt{-1} \gamma_{i}} \log \left(\frac{t}{\epsilon} e^{\sqrt{-1} \theta_{i}}\right) \in U_{d_{i}}$ for all $\epsilon \in \mathcal{E}_{i}$, all $t \in \mathcal{T}$ for $|t|>\mu_{\mathcal{T}}$. We consider the truncated Laplace transform (introduced in [18])

$$
\varphi_{j, U_{d_{i}}, \mathcal{E}_{i}, \gamma_{i}, \sigma^{\prime \prime}, \theta_{i}}(t, \epsilon)=\int_{0}^{e^{\sqrt{-1} \gamma_{i}} \log \left(\frac{t}{\epsilon} e^{\sqrt{-1} \theta_{i}}\right) /\left(\sigma^{\prime \prime} \zeta(b)\right)} V_{j, U_{d_{i}}, \mathcal{E}_{i}}(\tau, \epsilon) \exp \left(-\frac{t \tau}{\epsilon}\right) d \tau
$$

where the integration is made along the segment $\left[0, e^{\sqrt{-1} \gamma_{i}} \log \left(\frac{t}{\epsilon} e^{\sqrt{-1} \theta_{i}}\right) /\left(\sigma^{\prime \prime} \zeta(b)\right]\right.$, for all $t \in \mathcal{T}$, all $\epsilon \in \mathcal{E}_{i}$. 
The following theorem is crucial in order to establish our main result (Theorem 2) that will be stated in the next section. In this theorem we construct by means of Laplace transforms and truncated Laplace transforms a family of bounded holomorphic functions $X_{U_{d_{i}}, \mathcal{E}_{i}}(t, z, \epsilon)$ on products $(\mathcal{T} \cap\{|t|>h\}) \times D(0, \delta / 2) \times \mathcal{E}_{i}$, for $h>0$ large enough and $\delta>0$ small enough, for all $0 \leq i \leq \nu-1$, whose differences

$$
\Delta_{i}(\epsilon)=X_{U_{d_{i+1}}, \mathcal{E}_{i+1}}(t, z, \epsilon)-X_{U_{d_{i}}, \mathcal{E}_{i}}(t, z, \epsilon)
$$

satisfy exponential and super-exponential flatness estimates on the intersections $\mathcal{E}_{i+1} \cap \mathcal{E}_{i}$. The sequence of functions $\Delta_{i}(\epsilon), 0 \leq i \leq \nu-1$, viewed as functions from $\mathcal{E}_{i}$ into the Banach space $\mathbb{E}$ of bounded holomorphic functions on $(\mathcal{T} \cap\{|t|>h\}) \times D(0, \delta / 2)$ equipped with the supremum norm will be called a (Banach valued) cocycle according to the terminology of [26].

Theorem 1 We make the following assumptions.

There exists $\sigma^{\prime}>\tilde{\sigma}^{\prime}$ such that, for all $\underline{k} \in \mathcal{A}_{1}$, we have

$$
S \geq k_{1}+b k_{0}+\frac{k_{2} b}{\sigma^{\prime}} \quad, \quad S>k_{1},
$$

and for all $l \in \mathcal{A}_{2}$, we have

$$
l \geq 2 .
$$

Then, there exist constants $I>0$ and $\delta>0$ (independent of $\epsilon$ ) such that if we assume that for all $d_{i} \in(-\pi / 2, \pi / 2)$,

$$
\sum_{j=0}^{S-1-h}\left\|V_{j+h}(\tau, \epsilon)\right\|_{0, \epsilon, \tilde{\sigma}, 0, \Omega_{\tau_{0}, \epsilon_{0}}} \frac{\delta^{j}}{j !} \leq I \quad, \quad \sum_{j=0}^{S-1-h}\left\|V_{j+h, U_{d_{i}}, \mathcal{E}_{i}}(\tau, \epsilon)\right\|_{0, \epsilon, \tilde{\sigma}, 0, \Omega\left(d_{i}, \mathcal{E}_{i}\right)} \frac{\delta^{j}}{j !} \leq I,
$$

for all $0 \leq h \leq S-1$, for all $\epsilon \in \mathcal{E}_{i}$ and for all $d_{i} \in[-\pi,-\pi / 2) \cup(\pi / 2, \pi]$,

$$
\sum_{j=0}^{S-1-h}\left\|V_{j+h}(\tau, \epsilon)\right\|_{0, \epsilon, \tilde{\sigma}, \tilde{\sigma}^{\prime}, \Omega_{\tau_{0}, \epsilon_{0}}} \frac{\delta^{j}}{j !} \leq I \quad, \quad \sum_{j=0}^{S-1-h}\left\|V_{j+h, U_{d_{i}}, \mathcal{E}_{i}}(\tau, \epsilon)\right\|_{0, \epsilon, \tilde{\sigma}, \tilde{\sigma}^{\prime}, \Omega\left(d_{i}, \mathcal{E}_{i}\right)} \frac{\delta^{j}}{j !} \leq I,
$$

for all $0 \leq h \leq S-1$, for all $\epsilon \in \mathcal{E}_{i}$, there exists a family of holomorphic and bounded functions $X_{U_{d_{i}}, \mathcal{E}_{i}}(t, z, \epsilon), 0 \leq i \leq \nu-1$, on the product $\left(\mathcal{T} \cap\left\{|t|>\mu_{\mathcal{T}}\right\}\right) \times D(0, \delta / 2) \times \mathcal{E}_{i}$ with the following properties:

1) If $d_{i} \in(-\pi / 2, \pi / 2)$, then $X_{U_{d_{i}}, \mathcal{E}_{i}}(t, z, \epsilon)$ is the solution of the equation (100) with initial data

$$
\left(\partial_{z}^{j} X_{U_{d_{i}}, \mathcal{E}_{i}}\right)(t, 0, \epsilon)=\varphi_{j, U_{d_{i}}, \mathcal{E}_{i}}(t, \epsilon) \quad, \quad 0 \leq j \leq S-1 .
$$

2) If $d_{i} \in[-\pi,-\pi / 2) \cup(\pi / 2, \pi]$, then $X_{U_{d_{i}}, \mathcal{E}_{i}}(t, z, \epsilon)$ satisfies

$$
\left(\partial_{z}^{j} X_{U_{d_{i}}, \mathcal{E}_{i}}\right)(t, 0, \epsilon)=\varphi_{j, U_{d_{i}}, \mathcal{E}_{i}, \gamma_{i}, \sigma^{\prime \prime}, \theta_{i}}(t, \epsilon) \quad, \quad 0 \leq j \leq S-1 .
$$

3) For all $0 \leq i \leq \nu-1$, there exist constants $h \geq \mu_{\mathcal{T}}, K_{i}, M_{i}>0$ such that

$$
\sup _{t \in \mathcal{T} \cap\{|t|>h\}, z \in D(0, \delta / 2)}\left|X_{U_{d_{i+1}, \mathcal{E}_{i+1}}}(t, z, \epsilon)-X_{U_{d_{i}, \mathcal{E}_{i}}}(t, z, \epsilon)\right| \leq K_{i} e^{-\frac{M_{i}}{|\epsilon|}}
$$


for all $\epsilon \in \mathcal{E}_{i} \cap \mathcal{E}_{i+1}$ where by convention $\mathcal{E}_{\nu}=\mathcal{E}_{0}, d_{\nu}=d_{0}$.

4) If $d_{i+1}, d_{i} \in[-\pi,-\pi / 2) \cup(\pi / 2, \pi]$ and if moreover $U_{d_{i}} \cap U_{d_{i+1}} \neq \emptyset$, then there exist constants $h \geq \mu_{\mathcal{T}}, K_{i}, M_{i}>0$ and $L_{i}>1$ with

$$
\sup _{t \in \mathcal{T} \cap\{|t|>h\}, z \in D(0, \delta / 2)}\left|X_{U_{d_{i+1}, \mathcal{E}_{i+1}}}(t, z, \epsilon)-X_{U_{d_{i}, \mathcal{E}_{i}}}(t, z, \epsilon)\right| \leq K_{i} \exp \left(-\frac{M_{i}}{|\epsilon|} \log \frac{L_{i}}{|\epsilon|}\right)
$$

for all $\epsilon \in \mathcal{E}_{i} \cap \mathcal{E}_{i+1}$.

Proof If $d_{i} \in(-\pi / 2, \pi / 2)$, we consider the solution $X_{U_{d_{i}}, \mathcal{E}_{i}}(t, z, \epsilon)$ of the equation (100) with initial data

$$
\left(\partial_{z}^{j} X_{U_{d_{i}}, \mathcal{E}_{i}}\right)(t, 0, \epsilon)=\varphi_{j, U_{d_{i}}, \mathcal{E}_{i}}(t, \epsilon) \quad, \quad 0 \leq j \leq S-1 .
$$

constructed as in Proposition 7. Recall that the function $X_{U_{d_{i}}, \mathcal{E}_{i}}(t, z, \epsilon)$ can be written as a Laplace transform

$$
X_{U_{d_{i}}, \mathcal{E}_{i}}(t, z, \epsilon)=\int_{L_{\gamma_{i}}} V_{U_{d_{i}}, \mathcal{E}_{i}}(\tau, z, \epsilon) \exp \left(-\frac{t \tau}{\epsilon}\right) d \tau
$$

where $V_{U_{d_{i}}, \mathcal{E}_{i}}(\tau, z, \epsilon)$ is a holomorphic function on the domain $\left(U_{d_{i}} \cup D\left(0, \tau_{0}\right)\right) \times D(0, \delta / 2) \times \mathcal{E}_{i}$ and satisfies the following estimates: there exists a constant $C_{\Omega\left(d_{i}, \mathcal{E}_{i}\right)}>0$ (independent of $\epsilon$ ) such that

$$
\left|V_{U_{d_{i}}, \mathcal{E}_{i}}(\tau, z, \epsilon)\right| \leq C_{\Omega\left(d_{i}, \mathcal{E}_{i}\right)}\left(1+\frac{|\tau|^{2}}{|\epsilon|^{2}}\right)^{-1} \exp \left(\frac{\sigma \zeta(b)}{|\epsilon|}|\tau|\right)
$$

for all $(\tau, z, \epsilon) \in\left(U_{d_{i}} \cup D\left(0, \tau_{0}\right)\right) \times D(0, \delta / 2) \times \mathcal{E}_{i}$, for some $\sigma>\tilde{\sigma}$. Moreover, the function $V_{U_{d_{i}}}, \mathcal{E}_{i}(\tau, z, \epsilon)$ is the analytic continuation of a function $V(\tau, z, \epsilon)$ which is holomorphic on a punctured polydisc $D\left(0, \tau_{0}\right) \times D(0, \delta / 2) \times\left(D\left(0, \epsilon_{0}\right) \backslash\{0\}\right)$ and fulfills the next estimates: there exists a constant $C_{\Omega_{\tau_{0}, \epsilon_{0}}}>0$ (independent of $\epsilon$ ) such that

$$
|V(\tau, z, \epsilon)| \leq C_{\Omega_{\tau_{0}, \epsilon_{0}}}\left(1+\frac{|\tau|^{2}}{|\epsilon|^{2}}\right)^{-1} \exp \left(\frac{\sigma \zeta(b)}{|\epsilon|}|\tau|\right)
$$

for all $\tau \in D\left(0, \tau_{0}\right)$, all $z \in D(0, \delta / 2)$ and all $\epsilon \in D\left(0, \epsilon_{0}\right) \backslash\{0\}$.

If $d_{i} \in[-\pi,-\pi / 2) \cup(\pi / 2, \pi]$, we consider the following Cauchy problem

$$
\begin{array}{r}
\partial_{z}^{S} V_{U_{d_{i}}, \mathcal{E}_{i}}(\tau, z, \epsilon) \\
=\sum_{\underline{k}=\left(s, k_{0}, k_{1}, k_{2}\right) \in \mathcal{A}_{1}} \frac{(-1)^{k_{0}} b_{\underline{k}}(z, \epsilon)}{(a-\tau) \epsilon^{1+s+k_{0}} s !} \int_{0}^{\tau}(\tau-h)^{s} \exp \left(-k_{2} h\right) h^{k_{0}} \partial_{z}^{k_{1}} V_{U_{d_{i}}, \mathcal{E}_{i}}(h, z, \epsilon) d h \\
+\sum_{l \in \mathcal{A}_{2}} \frac{c_{l}(z, \epsilon)}{a-\tau} V_{U_{d_{i}}, \mathcal{E}_{i}}^{* l}(\tau, z, \epsilon)
\end{array}
$$

with the given initial conditions

$$
\left(\partial_{z}^{j} V_{U_{d_{i}}, \mathcal{E}_{i}}\right)(\tau, 0, \epsilon)=V_{j, U_{d_{i}}, \mathcal{E}_{i}}(\tau, \epsilon) \quad, \quad 0 \leq j \leq S-1
$$

for all $\tau \in D\left(0, \tau_{0}\right) \cup U_{d_{i}}, z \in D(0, \delta / 2)$ and $\epsilon \in \mathcal{E}_{i}$. Gathering the assumptions (115), (116) and (118) we deduce that the conditions (74), (75) and (76) from Proposition 6, 1) are fulfilled for the problem (126), (127). We get that the problem (126), (127) has a unique solution 
$V_{U_{d_{i}}, \mathcal{E}_{i}}(\tau, z, \epsilon)$ that belongs to the space $S G\left(\epsilon, \sigma, \sigma^{\prime}, \delta, \Omega\left(d_{i}, \mathcal{E}_{i}\right)\right)$ for $\sigma>\tilde{\sigma}$ and $\sigma^{\prime}>\tilde{\sigma}^{\prime}$. In particular, $V_{U_{d_{i}}, \mathcal{E}_{i}}(\tau, z, \epsilon)$ defines a holomorphic function on $\left(U_{d_{i}} \cup D\left(0, \tau_{0}\right)\right) \times D(0, \delta / 2) \times \mathcal{E}_{i}$, coincides with $V(\tau, z, \epsilon)$ on $D\left(0, \tau_{0}\right) \times D(0, \delta / 2) \times \mathcal{E}$ and satisfies the estimates

$$
\left|V_{U_{d_{i}}, \mathcal{E}_{i}}(\tau, z, \epsilon)\right| \leq C_{\Omega\left(d_{i}, \mathcal{E}_{i}\right)}\left(1+\frac{|\tau|^{2}}{|\epsilon|^{2}}\right)^{-1} \exp \left(\frac{\sigma \zeta(b)}{|\epsilon|}|\tau|+\exp \left(\sigma^{\prime} \zeta(b)|\tau|\right)\right)
$$

for all $(\tau, z, \epsilon) \in\left(U_{d_{i}} \cup D\left(0, \tau_{0}\right)\right) \times D(0, \delta / 2) \times \mathcal{E}_{i}$, due to Proposition 1 .

Now, we define the truncated Laplace transform

$$
X_{U_{d_{i}}, \mathcal{E}_{i}}(t, z, \epsilon)=\int_{0}^{e^{\sqrt{-1} \gamma_{i}} \log \left(\frac{t}{\epsilon} e^{\sqrt{-1} \theta_{i}}\right) /\left(\sigma^{\prime \prime} \zeta(b)\right)} V_{U_{d_{i}}, \mathcal{E}_{i}}(\tau, z, \epsilon) \exp \left(-\frac{t \tau}{\epsilon}\right) d \tau
$$

for all $(t, z, \epsilon) \in\left(\mathcal{T} \cap\left\{|t|>\mu_{\mathcal{T}}\right\}\right) \times D(0, \delta / 2) \times \mathcal{E}_{i}$, where $\sigma^{\prime \prime}>\sigma^{\prime}>\tilde{\sigma}^{\prime}$ and $\gamma_{i}$ are chosen as in (114).

In order to provide estimates for the differences of the functions $X_{U_{d_{i}}, \mathcal{E}_{i}}(t, z, \epsilon)$, we consider several cases.

Lemma 10 If $d_{i}, d_{i+1} \in(-\pi / 2, \pi / 2)$, then there exist constants $h \geq \mu_{\mathcal{T}}, K_{i}, M_{i}>0$ such that

$$
\sup _{t \in \mathcal{T} \cap\{|t|>h\}, z \in D(0, \delta / 2)}\left|X_{U_{d_{i+1}, \mathcal{E}_{i+1}}}(t, z, \epsilon)-X_{U_{d_{i}, \mathcal{E}_{i}}}(t, z, \epsilon)\right| \leq K_{i} e^{-\frac{M_{i}}{|\epsilon|}}
$$

for all $\epsilon \in \mathcal{E}_{i} \cap \mathcal{E}_{i+1}$.

Proof Let $i$ an integer with $0 \leq i \leq \nu-1$ such that $d_{i}, d_{i+1} \in(-\pi / 2, \pi / 2)$. From the fact that $\tau \mapsto V(\tau, z, \epsilon)$ is holomorphic on $D\left(0, \tau_{0}\right)$ for all $(z, \epsilon) \in D(0, \delta / 2) \times\left(D\left(0, \epsilon_{0}\right) \backslash\{0\}\right)$, the integral of $\tau \mapsto V(\tau, z, \epsilon) \exp (-t \tau / \epsilon)$ along the union of a segment starting from 0 to $\left(\tau_{0} / 2\right) e^{\sqrt{-1} \gamma_{i+1}}$, an arc of circle with radius $\tau_{0} / 2$ connecting $\left(\tau_{0} / 2\right) e^{\sqrt{-1} \gamma_{i+1}}$ and $\left(\tau_{0} / 2\right) e^{\sqrt{-1} \gamma_{i}}$ and a segment starting from $\left(\tau_{0} / 2\right) e^{\sqrt{-1} \gamma_{i}}$ to 0 , is equal to zero. Therefore, using the representation (123), we can rewrite the difference $X_{U_{d_{i+1}}, \mathcal{E}_{i+1}}-X_{U_{d_{i}}, \mathcal{E}_{i}}$ as a sum of three integrals,

$$
\begin{aligned}
X_{U_{d_{i+1}}, \mathcal{E}_{i+1}}(t, z, \epsilon)-X_{U_{d_{i}},} \mathcal{E}_{i}(t, z, \epsilon) & =\int_{L_{\tau_{0} / 2, \gamma_{i+1}}} V_{U_{d_{i+1}}, \mathcal{E}_{i+1}}(\tau, z, \epsilon) e^{-\frac{t \tau}{\epsilon}} d \tau \\
& -\int_{L_{\tau_{0} / 2, \gamma_{i}}} V_{U_{d_{i}}, \mathcal{E}_{i}}(\tau, z, \epsilon) e^{-\frac{t \tau}{\epsilon}} d \tau+\int_{C\left(\tau_{0} / 2, \gamma_{i}, \gamma_{i+1}\right)} V(\tau, z, \epsilon) e^{-\frac{t \tau}{\epsilon}} d \tau
\end{aligned}
$$

where $L_{\tau_{0} / 2, \gamma_{i}}=\left[\tau_{0} / 2,+\infty\right) e^{\sqrt{-1} \gamma_{i}}, L_{\tau_{0} / 2, \gamma_{i+1}}=\left[\tau_{0} / 2,+\infty\right) e^{\sqrt{-1} \gamma_{i+1}}$ and $C\left(\tau_{0} / 2, \gamma_{i}, \gamma_{i+1}\right)$ is an arc of circle with radius $\tau_{0} / 2$ connecting $\left(\tau_{0} / 2\right) e^{\sqrt{-1} \gamma_{i}}$ with $\left(\tau_{0} / 2\right) e^{\sqrt{-1} \gamma_{i+1}}$ with a well chosen orientation.

We give estimates for $I_{1}=\left|\int_{L_{\tau_{0} / 2, \gamma_{i+1}}} V_{U_{d_{i+1}}, \mathcal{E}_{i+1}}(\tau, z, \epsilon) e^{-\frac{t \tau}{\epsilon}} d \tau\right|$. By construction, the direction $\gamma_{i+1}$ (which depends on $\epsilon, t$ ) is chosen in such a way that $\cos \left(\gamma_{i+1}+\arg (t)-\arg (\epsilon)\right) \geq \delta_{1}$, for all $\epsilon \in \mathcal{E}_{i+1} \cap \mathcal{E}_{i}$, all $t \in \mathcal{T} \cap\left\{|t|>\mu_{\mathcal{T}}\right\}$, for some fixed $\delta_{1}>0$. From the estimates (124), we get

$$
\begin{aligned}
I_{1} \leq \int_{\tau_{0} / 2}^{+\infty} C_{\Omega\left(d_{i+1}, \mathcal{E}_{i+1}\right)}(1 & \left.+\frac{r^{2}}{|\epsilon|^{2}}\right)^{-1} e^{\frac{\sigma \zeta(b) r}{|\epsilon|}} e^{-\frac{r|t|}{|\epsilon|} \cos \left(\gamma_{i+1}+\arg (t)-\arg (\epsilon)\right)} d r \\
\leq & \int_{\tau_{0} / 2}^{+\infty} C_{\Omega\left(d_{i+1}, \mathcal{E}_{i+1}\right)} e^{\left(\sigma \zeta(b)-\delta_{1}|t|\right) \frac{r}{|\epsilon|}} d r \\
\quad & \frac{C_{\Omega\left(d_{i+1}, \mathcal{E}_{i+1}\right)}|\epsilon|}{\delta_{1}|t|-\sigma \zeta(b)} e^{-\left(\delta_{1}|t|-\sigma \zeta(b)\right) \frac{\tau_{0}}{2} \frac{1}{|\epsilon|}} \leq \frac{C_{\Omega\left(d_{i+1}, \mathcal{E}_{i+1}\right)} \epsilon_{0}}{\delta_{2}} e^{-\frac{\delta_{2} \tau_{0} / 2}{|\epsilon|}}
\end{aligned}
$$


for all $t \in \mathcal{T} \cap\left\{|t|>\mu_{\mathcal{T}}\right\}$, with $|t| \geq\left(\delta_{2}+\sigma \zeta(b)\right) / \delta_{1}$, for some $\delta_{2}>0$, and for all $\epsilon \in \mathcal{E}_{i+1} \cap \mathcal{E}_{i}$.

We give also estimates for $I_{2}=\left|\int_{L_{\tau_{0} / 2, \gamma_{i}}} V_{U_{d_{i}}, \mathcal{E}_{i}}(\tau, z, \epsilon) e^{-\frac{t \tau}{\epsilon}} d \tau\right|$. By construction, the direction $\gamma_{i}$ (which depends on $\left.\epsilon, t\right)$ is chosen in such a way that there exists a fixed $\delta_{1}>0$ with $\cos \left(\gamma_{i}+\right.$ $\arg (t)-\arg (\epsilon)) \geq \delta_{1}$, for all $\epsilon \in \mathcal{E}_{i+1} \cap \mathcal{E}_{i}$, all $t \in \mathcal{T} \cap\left\{|t|>\mu_{\mathcal{T}}\right\}$. From the estimates (124), we deduce as above that

$$
I_{2} \leq \frac{C_{\Omega\left(d_{i}, \mathcal{E}_{i}\right)} \epsilon_{0}}{\delta_{2}} e^{-\frac{\delta_{2} \tau_{0} / 2}{|\epsilon|}}
$$

for all $t \in \mathcal{T} \cap\left\{|t|>\mu_{\mathcal{T}}\right\}$, with $|t| \geq\left(\delta_{2}+\sigma \zeta(b)\right) / \delta_{1}$, for some $\delta_{2}>0$, and for all $\epsilon \in \mathcal{E}_{i+1} \cap \mathcal{E}_{i}$.

Finally, we get estimates for $I_{3}=\left|\int_{C\left(\tau_{0} / 2, \gamma_{i}, \gamma_{i+1}\right)} V(\tau, z, \epsilon) e^{-\frac{t \tau}{\epsilon}} d \tau\right|$. From the estimates (125), we have

$$
I_{3} \leq\left|\int_{\gamma_{i}}^{\gamma_{i+1}} C_{\Omega_{\tau_{0}, \epsilon_{0}}}\left(1+\frac{\left(\tau_{0} / 2\right)^{2}}{|\epsilon|^{2}}\right)^{-1} e^{\frac{\sigma \zeta(b) \tau_{0}}{2|\epsilon|}} e^{-\frac{|t| \tau_{0}}{2|\epsilon|} \cos (\theta+\arg (t)-\arg (\epsilon))} \frac{\tau_{0}}{2} d \theta\right|
$$

By construction, the arc of circle $C\left(\tau_{0} / 2, \gamma_{i}, \gamma_{i+1}\right)$ is chosen in such a way that that $\cos (\theta+$ $\arg (t)-\arg (\epsilon)) \geq \delta_{1}$, for all $\theta \in\left[\gamma_{i}, \gamma_{i+1}\right]$ (if $\left.\gamma_{i}<\gamma_{i+1}\right)$ ), $\theta \in\left[\gamma_{i+1}, \gamma_{i}\right]$ (if $\left.\gamma_{i+1}<\gamma_{i}\right)$ ), for all $t \in \mathcal{T}$, all $\epsilon \in \mathcal{E}_{i} \cap \mathcal{E}_{i+1}$. From (134), we deduce that

$$
I_{3} \leq\left|\gamma_{i+1}-\gamma_{i}\right| C_{\Omega_{\tau_{0}, \epsilon_{0}}} \frac{\tau_{0}}{2} e^{-\left(\left(\delta_{1}|t|-\sigma \zeta(b)\right) \frac{\tau_{0}}{2}\right) \frac{1}{|\epsilon|}} \leq\left|\gamma_{i+1}-\gamma_{i}\right| C_{\Omega_{\tau_{0}, \epsilon_{0}}} \frac{\tau_{0}}{2} e^{-\frac{\delta_{2} \tau_{0}}{2|\epsilon|}}
$$

for all $t \in \mathcal{T} \cap\left\{|t|>\mu_{\mathcal{T}}\right\}$, with $|t| \geq\left(\delta_{2}+\sigma \zeta(b)\right) / \delta_{1}$, for some $\delta_{2}>0$, and for all $\epsilon \in \mathcal{E}_{i+1} \cap \mathcal{E}_{i}$.

Finally, collecting the inequalities (131), (132), (133), (135) we obtain that the estimates (130) hold.

Lemma 11 If $d_{i} \in(-\pi / 2, \pi / 2)$ and $d_{i+1} \in[-\pi,-\pi / 2) \cup(\pi / 2, \pi]$ or $d_{i+1} \in(-\pi / 2, \pi / 2)$ and $d_{i} \in[-\pi,-\pi / 2) \cup(\pi / 2, \pi]$ then there exist constants $h \geq \mu_{\mathcal{T}}, K_{i}, M_{i}>0$ such that

$$
\sup _{t \in \mathcal{T} \cap\{|t|>h\}, z \in D(0, \delta / 2)}\left|X_{U_{d_{i+1}}, \mathcal{E}_{i+1}}(t, z, \epsilon)-X_{U_{d_{i}}, \mathcal{E}_{i}}(t, z, \epsilon)\right| \leq K_{i} e^{-\frac{M_{i}}{|\epsilon|}}
$$

for all $\epsilon \in \mathcal{E}_{i} \cap \mathcal{E}_{i+1}$.

Proof Assume that $d_{i} \in(-\pi / 2, \pi / 2)$ and $d_{i+1} \in[-\pi,-\pi / 2) \cup(\pi / 2, \pi]$. The other alternative can be handled in a similar way. We know that $\tau \mapsto V(\tau, z, \epsilon)$ is holomorphic on $D\left(0, \tau_{0}\right)$ for all $(z, \epsilon) \in D(0, \delta / 2) \times\left(D\left(0, \epsilon_{0}\right) \backslash\{0\}\right)$. Therefore, the integral of $\tau \mapsto V(\tau, z, \epsilon) \exp (-t \tau / \epsilon)$ along the segment

$$
\left[0, \frac{\tau_{0}}{2} e^{\sqrt{-1}\left(\gamma_{i+1}+\arg \left(\log \left(\frac{t}{\epsilon} e^{\sqrt{-1} \theta_{i+1}}\right)\right)\right)}\right]
$$

followed by an arc of circle with radius $\tau_{0} / 2$ connecting

$$
\frac{\tau_{0}}{2} e^{\sqrt{-1}\left(\gamma_{i+1}+\arg \left(\log \left(\frac{t}{\epsilon} e^{\sqrt{-1} \theta_{i+1}}\right)\right)\right)} \text { and } \frac{\tau_{0}}{2} e^{\sqrt{-1}\left(\gamma_{i}+\arg \left(\log \left(\frac{t}{\epsilon} e^{\sqrt{-1} \theta_{i}}\right)\right)\right)}
$$

and along the segment

$$
\left[\frac{\tau_{0}}{2} e^{\sqrt{-1}\left(\gamma_{i}+\arg \left(\log \left(\frac{t}{\epsilon} e^{\sqrt{-1} \theta_{i}}\right)\right)\right)}, 0\right]
$$


is equal to zero. So that, using the representations (123) and (129) we can rewrite the difference $X_{U_{d_{i+1}}, \mathcal{E}_{i+1}}-X_{U_{d_{i}}, \mathcal{E}_{i}}$ as a sum of three integrals,

$$
\begin{aligned}
X_{U_{d_{i+1}}, \mathcal{E}_{i+1}}(t, z, \epsilon) & -X_{U_{d_{i}}, \mathcal{E}_{i}}(t, z, \epsilon)=\int_{L_{\tau_{0} / 2, \gamma_{i+1}, t}} V_{U_{d_{i+1}}, \mathcal{E}_{i+1}}(\tau, z, \epsilon) e^{-\frac{t \tau}{\epsilon}} d \tau \\
& -\int_{L_{\tau_{0} / 2, \gamma_{i}, t, \infty}} V_{U_{d_{i}}, \mathcal{E}_{i}}(\tau, z, \epsilon) e^{-\frac{t \tau}{\epsilon}} d \tau+\int_{C\left(\tau_{0} / 2, \gamma_{i}, \gamma_{i+1}, t\right)} V(\tau, z, \epsilon) e^{-\frac{t \tau}{\epsilon}} d \tau
\end{aligned}
$$

where

$$
\begin{aligned}
& L_{\tau_{0} / 2, \gamma_{i}, t, \infty}=\left[\frac{\tau_{0}}{2},+\infty\right) e^{\sqrt{-1}\left(\gamma_{i}+\arg \left(\log \left(\frac{t}{\epsilon} e^{\sqrt{-1} \theta_{i}}\right)\right)\right)}, \\
& L_{\tau_{0} / 2, \gamma_{i+1}, t}=\left[\frac{\tau_{0}}{2} e^{\sqrt{-1}\left(\gamma_{i+1}+\arg \left(\log \left(\frac{t}{\epsilon} e^{\sqrt{-1} \theta_{i+1}}\right)\right)\right)}, e^{\sqrt{-1} \gamma_{i+1}} \frac{\log \left(\frac{t}{\epsilon} e^{\sqrt{-1} \theta_{i+1}}\right)}{\sigma^{\prime \prime} \zeta(b)}\right]
\end{aligned}
$$

and $C\left(\tau_{0} / 2, \gamma_{i}, \gamma_{i+1}, t\right)$ is an arc of circle with radius $\tau_{0} / 2$ connecting

$$
\frac{\tau_{0}}{2} e^{\sqrt{-1}\left(\gamma_{i}+\arg \left(\log \left(\frac{t}{\epsilon} e^{\sqrt{-1} \theta_{i}}\right)\right)\right)} \text { with } \frac{\tau_{0}}{2} e^{\sqrt{-1}\left(\gamma_{i+1}+\arg \left(\log \left(\frac{t}{\epsilon} e^{\sqrt{-1} \theta_{i+1}}\right)\right)\right)}
$$

with a well chosen orientation.

We show estimates for the first integral

$$
I_{1}=\left|\int_{L_{\tau_{0} / 2, \gamma_{i+1}, t}} V_{U_{d_{i+1}}, \mathcal{E}_{i+1}}(\tau, z, \epsilon) e^{-\frac{t \tau}{\epsilon}} d \tau\right|
$$

From the estimates (128), we deduce that

$$
\begin{aligned}
I_{1} \leq \int_{\tau_{0} / 2}^{\frac{\left|\log \left(\frac{t}{\epsilon} e^{\sqrt{-1}} \theta_{i+1}\right)\right|}{\sigma^{\prime \prime} \zeta(b)}} C_{\Omega\left(d_{i+1}, \mathcal{E}_{i+1}\right)}\left(1+\frac{h^{2}}{|\epsilon|^{2}}\right)^{-1} \exp \left(\frac{\sigma \zeta(b)}{|\epsilon|} h+\exp \left(\sigma^{\prime} \zeta(b) h\right)\right) \\
\times \exp \left(-\operatorname{Re}\left(\frac{t}{\epsilon} h \exp \left(\sqrt{-1}\left(\gamma_{i+1}+\arg \left(\log \left(\frac{t}{\epsilon} e^{\sqrt{-1} \theta_{i+1}}\right)\right)\right)\right)\right)\right) d h
\end{aligned}
$$

We know that

$$
\begin{aligned}
\left|\log \left(\frac{t}{\epsilon} e^{\sqrt{-1} \theta_{i+1}}\right)\right|=\left(\left(\log \left|\frac{t}{\epsilon}\right|\right)^{2}+\right. & \left.\left(\arg (t)-\arg (\epsilon)+\theta_{i+1}\right)^{2}\right)^{1 / 2} \\
& \arg \left(\log \left(\frac{t}{\epsilon} e^{\sqrt{-1} \theta_{i+1}}\right)\right)=\arctan \left(\frac{\arg (t)-\arg (\epsilon)+\theta_{i+1}}{\log \left|\frac{t}{\epsilon}\right|}\right)
\end{aligned}
$$

for all $t \in \mathcal{T}$ with $|t|>\mu_{\mathcal{T}}$ provided that $\mu_{\mathcal{T}}>0$ is large enough, all $\epsilon \in \mathcal{E}_{i} \cap \mathcal{E}_{i+1}$. Therefore, we can choose $\mu_{\mathcal{T}}>0$ large enough such that there exists a constant $0<C_{14}<\sigma^{\prime \prime} / \sigma^{\prime}$ (depending on $\mu_{\mathcal{T}}$ ) with

$$
\left|\log \left(\frac{t}{\epsilon} e^{\sqrt{-1} \theta_{i+1}}\right)\right| \leq C_{14} \log \left|\frac{t}{\epsilon}\right|
$$

for all $t \in \mathcal{T} \cap\left\{|t|>\mu_{\mathcal{T}}\right\}$, all $\epsilon \in \mathcal{E}_{i} \cap \mathcal{E}_{i+1}$. Moreover, using (139) we deduce from (113) that there exists $\delta_{1}^{\prime}>0$ (which may be smaller than $\delta_{1}$ defined in (113)) with

$$
\begin{aligned}
\operatorname{Re}\left(\frac{t}{\epsilon} h \exp \right. & \left.\left(\sqrt{-1}\left(\gamma_{i+1}+\arg \left(\log \left(\frac{t}{\epsilon} e^{\sqrt{-1} \theta_{i+1}}\right)\right)\right)\right)\right) \\
& =\left|\frac{t}{\epsilon}\right| h \cos \left(\gamma_{i+1}+\arg (t)-\arg (\epsilon)+\arctan \left(\frac{\arg (t)-\arg (\epsilon)+\theta_{i+1}}{\log \left|\frac{t}{\epsilon}\right|}\right)\right) \geq \delta_{1}^{\prime}\left|\frac{t}{\epsilon}\right| h
\end{aligned}
$$


for all $t \in \mathcal{T} \cap\left\{|t|>\mu_{\mathcal{T}}\right\}$, all $\epsilon \in \mathcal{E}_{i} \cap \mathcal{E}_{i+1}$. From (138), (140) and (141) we get that

$$
\begin{aligned}
& I_{1} \leq \int_{\tau_{0} / 2}^{\frac{C_{14} \log \left|\frac{t}{\epsilon}\right|}{\sigma^{\prime \prime} \zeta(b)}} C_{\Omega\left(d_{i+1}, \mathcal{E}_{i+1}\right)} \exp \left(\frac{\sigma \zeta(b)}{|\epsilon|} h+\exp \left(\sigma^{\prime} \zeta(b) h\right)\right) \exp \left(-\delta_{1}^{\prime}\left|\frac{t}{\epsilon}\right| h\right) d h \\
& \leq C_{\Omega\left(d_{i+1}, \mathcal{E}_{i+1}\right)} \exp \left(\left|\frac{t}{\epsilon}\right|^{\frac{\sigma^{\prime} C_{14}}{\sigma^{\prime \prime}}}\right) \int_{\tau_{0} / 2}^{\frac{C_{14} \log \left|\frac{t}{\epsilon}\right|}{\sigma^{\prime \prime} \zeta(b)}} \exp \left(-\left(\delta_{1}^{\prime}|t|-\sigma \zeta(b)\right) \frac{h}{|\epsilon|}\right) d h \\
& =C_{\Omega\left(d_{i+1}, \mathcal{E}_{i+1}\right)} \exp \left(\left|\frac{t}{\epsilon}\right|^{\frac{\sigma^{\prime} C_{14}}{\sigma^{\prime \prime}}}\right) \frac{|\epsilon|}{\delta_{1}^{\prime}|t|-\sigma \zeta(b)} \\
& \times\left(\exp \left(-\left(\delta_{1}^{\prime}|t|-\sigma \zeta(b)\right) \frac{\tau_{0}}{2|\epsilon|}\right)-\exp \left(-\left(\delta_{1}^{\prime}|t|-\sigma \zeta(b)\right) \frac{C_{14} \log \left|\frac{t}{\epsilon}\right|}{\sigma^{\prime \prime} \zeta(b)|\epsilon|}\right)\right)
\end{aligned}
$$

Now, from the choice of the constant $C_{14}$ in (140), we can choose $0<\delta_{2}<\delta_{1}^{\prime} / 4$ such that

$$
|t|>\frac{\sigma \zeta(b)}{\delta_{1}^{\prime}-4 \delta_{2}}, \quad\left(\frac{|t|}{\epsilon_{0}}\right)^{\frac{\sigma^{\prime} C_{14}}{\sigma^{\prime \prime}}-1} \leq \delta_{2} \tau_{0}
$$

for all $t \in \mathcal{T} \cap\left\{|t| \geq \mu_{\mathcal{T}}\right\}$ (whenever $\mu_{\mathcal{T}}$ is large enough). Therefore, we deduce that

$$
\exp \left(\left|\frac{t}{\epsilon}\right| \frac{\sigma^{\prime} C_{14}}{\sigma^{\prime \prime}}\right) \exp \left(-\left(\delta_{1}^{\prime}|t|-\sigma \zeta(b)\right) \frac{\tau_{0}}{2|\epsilon|}\right) \leq \exp \left(-\delta_{2} \tau_{0} \frac{|t|}{|\epsilon|}\right) \leq \exp \left(-\delta_{2} \tau_{0} \frac{\mu_{\mathcal{T}}}{|\epsilon|}\right)
$$

for all $t \in \mathcal{T} \cap\left\{|t|>\mu_{\mathcal{T}}\right\}$, all $\epsilon \in \mathcal{E}_{i} \cap \mathcal{E}_{i+1}$. Gathering (142) and (144) yields

$$
I_{1} \leq \frac{C_{\Omega\left(d_{i+1}, \mathcal{E}_{i+1}\right)} \epsilon_{0}}{4 \delta_{2} \mu_{\mathcal{T}}} \exp \left(-\delta_{2} \tau_{0} \frac{\mu_{\mathcal{T}}}{|\epsilon|}\right)
$$

for all $t \in \mathcal{T} \cap\left\{|t|>\mu_{\mathcal{T}}\right\}$, all $\epsilon \in \mathcal{E}_{i} \cap \mathcal{E}_{i+1}$.

We give estimates for the second integral

$$
I_{2}=\left|\int_{L_{\tau_{0} / 2, \gamma_{i}, t, \infty}} V_{U_{d_{i}}, \mathcal{E}_{i}}(\tau, z, \epsilon) e^{-\frac{t \tau}{\epsilon}} d \tau\right| .
$$

From (124), we get that

$$
\begin{aligned}
I_{2} \leq \int_{\tau_{0} / 2}^{+\infty} C_{\Omega\left(d_{i}, \mathcal{E}_{i}\right)}\left(1+\frac{h^{2}}{|\epsilon|^{2}}\right)^{-1} & \exp \left(\frac{\sigma \zeta(b)}{|\epsilon|} h\right) \\
& \times \exp \left(-\operatorname{Re}\left(\frac{t}{\epsilon} h \exp \left(\sqrt{-1}\left(\gamma_{i}+\arg \left(\log \left(\frac{t}{\epsilon} e^{\sqrt{-1} \theta_{i}}\right)\right)\right)\right)\right)\right) d h
\end{aligned}
$$

for all $t \in \mathcal{T} \cap\left\{|t|>\mu_{\mathcal{T}}\right\}$, all $\epsilon \in \mathcal{E}_{i} \cap \mathcal{E}_{i+1}$. As before, with the help of (113) and (139), we get a constant $\delta_{1}^{\prime}>0$ (which can be smaller than $\delta_{1}$ from (113)) with

$$
\begin{aligned}
& \operatorname{Re}\left(\frac{t}{\epsilon} h \exp \left(\sqrt{-1}\left(\gamma_{i}+\arg \left(\log \left(\frac{t}{\epsilon} e^{\sqrt{-1} \theta_{i}}\right)\right)\right)\right)\right) \\
& \quad=\left|\frac{t}{\epsilon}\right| h \cos \left(\gamma_{i}+\arg (t)-\arg (\epsilon)+\arctan \left(\frac{\arg (t)-\arg (\epsilon)+\theta_{i}}{\log \left|\frac{t}{\epsilon}\right|}\right)\right) \geq \delta_{1}^{\prime}\left|\frac{t}{\epsilon}\right| h
\end{aligned}
$$


for all $t \in \mathcal{T} \cap\left\{|t|>\mu_{\mathcal{T}}\right\}$, all $\epsilon \in \mathcal{E}_{i} \cap \mathcal{E}_{i+1}$. Hence,

$$
\begin{aligned}
I_{2} \leq \int_{\tau_{0} / 2}^{+\infty} C_{\Omega\left(d_{i}, \mathcal{E}_{i}\right)} e^{\left(\sigma \zeta(b)-\delta_{1}^{\prime}|t|\right) \frac{r}{|\epsilon|}} d r & \\
& =\frac{C_{\Omega\left(d_{i}, \mathcal{E}_{i}\right)}|\epsilon|}{\delta_{1}^{\prime}|t|-\sigma \zeta(b)} e^{\left.-\left(\delta_{1}^{\prime}|t|-\sigma \zeta(b)\right) \frac{\tau_{0}}{2}\right) \frac{1}{|\epsilon|}} \leq \frac{C_{\Omega\left(d_{i}, \mathcal{E}_{i}\right)} \epsilon_{0}}{\delta_{2}} e^{-\frac{\delta_{2} \tau_{0} / 2}{|\epsilon|}}
\end{aligned}
$$

for all $t \in \mathcal{T} \cap\left\{|t|>\mu_{\mathcal{T}}\right\}$, with $|t| \geq\left(\delta_{2}+\sigma \zeta(b)\right) / \delta_{1}^{\prime}$, for some $\delta_{2}>0$, and for all $\epsilon \in \mathcal{E}_{i+1} \cap \mathcal{E}_{i}$.

Finally, we treat the third integral

$$
I_{3}=\left|\int_{C\left(\tau_{0} / 2, \gamma_{i}, \gamma_{i+1}, t\right)} V(\tau, z, \epsilon) e^{-\frac{t \tau}{\epsilon}} d \tau\right|
$$

From the estimates (125), we have

$$
I_{3} \leq\left|\int_{\gamma_{i}+\arg \left(\log \left(\frac{t}{\epsilon} e^{\sqrt{-1} \theta_{i}}\right)\right)}^{\gamma_{i+1}+\arg \left(\log \left(\frac{t}{\epsilon} e^{\sqrt{-1} \theta_{i+1}}\right)\right)} C_{\Omega_{\tau_{0}, \epsilon_{0}}}\left(1+\frac{\left(\tau_{0} / 2\right)^{2}}{|\epsilon|^{2}}\right)^{-1} e^{\frac{\sigma \zeta(b) \tau_{0}}{2|\epsilon|}} e^{-\frac{|t| \tau_{0}}{2|\epsilon|} \cos (\theta+\arg (t)-\arg (\epsilon))} \frac{\tau_{0}}{2} d \theta\right|
$$

Bearing in mind (139), the arc of circle $C\left(\tau_{0} / 2, \gamma_{i}, \gamma_{i+1}, t\right)$ is chosen in such a way that $\cos (\theta+$ $\arg (t)-\arg (\epsilon)) \geq \delta_{1}^{\prime}$, for some $0<\delta_{1}^{\prime}<\delta_{1}$ where $\delta_{1}$ is defined in (113), for all

$$
\begin{aligned}
& \theta \in\left[\gamma_{i}+\arg \left(\log \left(\frac{t}{\epsilon} e^{\sqrt{-1} \theta_{i}}\right)\right), \gamma_{i+1}+\arg \left(\log \left(\frac{t}{\epsilon} e^{\sqrt{-1} \theta_{i+1}}\right)\right)\right] \text { if } \gamma_{i}<\gamma_{i+1}, \\
& \theta \in\left[\gamma_{i+1}+\arg \left(\log \left(\frac{t}{\epsilon} e^{\sqrt{-1} \theta_{i+1}}\right)\right), \gamma_{i}+\arg \left(\log \left(\frac{t}{\epsilon} e^{\sqrt{-1} \theta_{i}}\right)\right)\right] \text { if } \gamma_{i+1}<\gamma_{i},
\end{aligned}
$$

for all $t \in \mathcal{T}$, all $\epsilon \in \mathcal{E}_{i} \cap \mathcal{E}_{i+1}$. From (149) and using (139) we deduce that

$$
I_{3} \leq\left(\left|\gamma_{i+1}-\gamma_{i}\right|+\pi\right) C_{\Omega_{\tau_{0}, \epsilon_{0}}} \frac{\tau_{0}}{2} e^{-\left(\left(\delta_{1}^{\prime}|t|-\sigma \zeta(b)\right) \frac{\tau_{0}}{2}\right) \frac{1}{|\epsilon|}} \leq\left(\left|\gamma_{i+1}-\gamma_{i}\right|+\pi\right) C_{\Omega_{\tau_{0}, \epsilon_{0}}} \frac{\tau_{0}}{2} e^{-\frac{\delta_{2} \tau_{0}}{2|\epsilon|}}
$$

for all $t \in \mathcal{T} \cap\{|t|>\mu \mathcal{T}\}$, with $|t| \geq\left(\delta_{2}+\sigma \zeta(b)\right) / \delta_{1}^{\prime}$, for some $\delta_{2}>0$, and for all $\epsilon \in \mathcal{E}_{i+1} \cap \mathcal{E}_{i}$.

Finally, by collecting (137), (145), (148), (150) we deduce that the estimates (136) hold.

Lemma 12 If $d_{i}, d_{i+1} \in[-\pi,-\pi / 2) \cup(\pi / 2, \pi]$, then there exist constants $h \geq \mu_{\mathcal{T}}, K_{i}, M_{i}>0$ such that

$$
\sup _{t \in \mathcal{T} \cap\{|t|>h\}, z \in D(0, \delta / 2)}\left|X_{U_{d_{i+1}}, \mathcal{E}_{i+1}}(t, z, \epsilon)-X_{U_{d_{i}}, \mathcal{E}_{i}}(t, z, \epsilon)\right| \leq K_{i} e^{-\frac{M_{i}}{|\epsilon|}}
$$

for all $\epsilon \in \mathcal{E}_{i} \cap \mathcal{E}_{i+1}$.

Proof Let $d_{i}, d_{i+1} \in[-\pi,-\pi / 2) \cup(\pi / 2, \pi]$. We know that $\tau \mapsto V(\tau, z, \epsilon)$ is holomorphic on $D\left(0, \tau_{0}\right)$ for all $(z, \epsilon) \in D(0, \delta / 2) \times\left(D\left(0, \epsilon_{0}\right) \backslash\{0\}\right)$. Therefore, the integral of $\tau \mapsto V(\tau, z, \epsilon) \exp (-t \tau / \epsilon)$ along the segment

$$
\left[0, \frac{\tau_{0}}{2} e^{\sqrt{-1}\left(\gamma_{i+1}+\arg \left(\log \left(\frac{t}{\epsilon} e^{\sqrt{-1} \theta_{i+1}}\right)\right)\right.}\right]
$$

followed by an arc of circle with radius $\tau_{0} / 2$ connecting

$$
\frac{\tau_{0}}{2} e^{\sqrt{-1}\left(\gamma_{i+1}+\arg \left(\log \left(\frac{t}{\epsilon} e^{\sqrt{-1} \theta_{i+1}}\right)\right)\right)} \text { and } \frac{\tau_{0}}{2} e^{\sqrt{-1}\left(\gamma_{i}+\arg \left(\log \left(\frac{t}{\epsilon} e^{\sqrt{-1} \theta_{i}}\right)\right)\right)}
$$


and along the segment

$$
\left[\frac{\tau_{0}}{2} e^{\sqrt{-1}\left(\gamma_{i}+\arg \left(\log \left(\frac{t}{\epsilon} e^{\sqrt{-1} \theta_{i}}\right)\right)\right)}, 0\right]
$$

is equal to zero. Hence, using the representation (129) we can rewrite the difference $X_{U_{d_{i+1}}, \mathcal{E}_{i+1}}$ $X_{U_{d_{i}}, \mathcal{E}_{i}}$ as a sum of three integrals,

$$
\begin{aligned}
X_{U_{d_{i+1}}, \mathcal{E}_{i+1}}(t, z, \epsilon)- & X_{U_{d_{i}}, \mathcal{E}_{i}}(t, z, \epsilon)=\int_{L_{\tau_{0} / 2, \gamma_{i+1}, t}} V_{U_{d_{i+1}}, \mathcal{E}_{i+1}}(\tau, z, \epsilon) e^{-\frac{t \tau}{\epsilon}} d \tau \\
& -\int_{L_{\tau_{0} / 2, \gamma_{i}, t}} V_{U_{d_{i}}, \mathcal{E}_{i}}(\tau, z, \epsilon) e^{-\frac{t \tau}{\epsilon}} d \tau+\int_{C\left(\tau_{0} / 2, \gamma_{i}, \gamma_{i+1}, t\right)} V(\tau, z, \epsilon) e^{-\frac{t \tau}{\epsilon}} d \tau
\end{aligned}
$$

where

$$
\begin{gathered}
L_{\tau_{0} / 2, \gamma_{i}, t}=\left[\frac{\tau_{0}}{2} e^{\sqrt{-1}\left(\gamma_{i}+\arg \left(\log \left(\frac{t}{\epsilon} e^{\sqrt{-1} \theta_{i}}\right)\right)\right)}, e^{\sqrt{-1} \gamma_{i}} \frac{\log \left(\frac{t}{\epsilon} e^{\sqrt{-1} \theta_{i}}\right)}{\sigma^{\prime \prime} \zeta(b)}\right], \\
L_{\tau_{0} / 2, \gamma_{i+1}, t}=\left[\frac{\tau_{0}}{2} e^{\sqrt{-1}\left(\gamma_{i+1}+\arg \left(\log \left(\frac{t}{\epsilon} e^{\sqrt{-1} \theta_{i+1}}\right)\right)\right)}, e^{\sqrt{-1} \gamma_{i+1}} \frac{\log \left(\frac{t}{\epsilon} e^{\sqrt{-1} \theta_{i+1}}\right)}{\sigma^{\prime \prime} \zeta(b)}\right]
\end{gathered}
$$

and $C\left(\tau_{0} / 2, \gamma_{i}, \gamma_{i+1}, t\right)$ is an arc of circle with radius $\tau_{0} / 2$ connecting

$$
\frac{\tau_{0}}{2} e^{\sqrt{-1}\left(\gamma_{i}+\arg \left(\log \left(\frac{t}{\epsilon} e^{\sqrt{-1} \theta_{i}}\right)\right)\right)} \text { with } \frac{\tau_{0}}{2} e^{\sqrt{-1}\left(\gamma_{i+1}+\arg \left(\log \left(\frac{t}{\epsilon} e^{\sqrt{-1} \theta_{i+1}}\right)\right)\right)}
$$

with a well chosen orientation.

Using the same arguments as in the proof of (145), we get a constant $\delta_{2}>0$ such that

$$
\begin{gathered}
\left|\int_{L_{\tau_{0} / 2, \gamma_{i+1}, t}} V_{U_{d_{i+1}}, \mathcal{E}_{i+1}}(\tau, z, \epsilon) e^{-\frac{t \tau}{\epsilon}} d \tau\right| \leq \frac{C_{\Omega\left(d_{i+1}, \mathcal{E}_{i+1}\right)} \epsilon_{0}}{4 \delta_{2} \mu_{\mathcal{T}}} \exp \left(-\delta_{2} \tau_{0} \frac{\mu_{\mathcal{T}}}{|\epsilon|}\right) \\
\left|\int_{L_{\tau_{0} / 2, \gamma_{i}, t}} V_{U_{d_{i}}, \mathcal{E}_{i}}(\tau, z, \epsilon) e^{-\frac{t \tau}{\epsilon}} d \tau\right| \leq \frac{C_{\Omega\left(d_{i}, \mathcal{E}_{i}\right)} \epsilon_{0}}{4 \delta_{2} \mu_{\mathcal{T}}} \exp \left(-\delta_{2} \tau_{0} \frac{\mu_{\mathcal{T}}}{|\epsilon|}\right)
\end{gathered}
$$

for all $t \in \mathcal{T} \cap\left\{|t|>\mu_{\mathcal{T}}\right\}$, all $\epsilon \in \mathcal{E}_{i} \cap \mathcal{E}_{i+1}$.

On the other hand, following a similar outline from the proof of (150) yields a constant $\delta_{2}>0$ with

$$
\left|\int_{C\left(\tau_{0} / 2, \gamma_{i}, \gamma_{i+1}, t\right)} V(\tau, z, \epsilon) e^{-\frac{t \tau}{\epsilon}} d \tau\right| \leq\left(\left|\gamma_{i+1}-\gamma_{i}\right|+\pi\right) C_{\Omega_{\tau_{0}, \epsilon_{0}}} \frac{\tau_{0}}{2} e^{-\frac{\delta_{2} \tau_{0}}{2|\epsilon|}}
$$

for all $t \in \mathcal{T} \cap\left\{|t|>\mu_{\mathcal{T}}\right\}$, with $|t| \geq\left(\delta_{2}+\sigma \zeta(b)\right) / \delta_{1}^{\prime}$, for all $\epsilon \in \mathcal{E}_{i+1} \cap \mathcal{E}_{i}$.

Finally, taking into account (152), (153), (154), we get the estimates (151).

Lemma 13 If $d_{i+1}, d_{i} \in[-\pi,-\pi / 2) \cup(\pi / 2, \pi]$ and if moreover $U_{d_{i}} \cap U_{d_{i+1}} \neq \emptyset$, then there exist constants $h \geq \mu_{\mathcal{T}}, K_{i}, M_{i}>0$ and $L_{i}>1$ such that

$$
\sup _{t \in \mathcal{T} \cap\{|t|>h\}, z \in D(0, \delta / 2)}\left|X_{U_{d_{i+1}, \mathcal{E}_{i+1}}}(t, z, \epsilon)-X_{U_{d_{i}, \mathcal{E}_{i}}}(t, z, \epsilon)\right| \leq K_{i} \exp \left(-\frac{M_{i}}{|\epsilon|} \log \frac{L_{i}}{|\epsilon|}\right)
$$

for all $\epsilon \in \mathcal{E}_{i} \cap \mathcal{E}_{i+1}$. 
Proof Let $d_{i+1}, d_{i} \in[-\pi,-\pi / 2) \cup(\pi / 2, \pi]$ and we assume moreover that $U_{d_{i}} \cap U_{d_{i+1}} \neq \emptyset$. In that case, the function $\tau \mapsto V(\tau, z, \epsilon)$ which is holomorphic on $D\left(0, \tau_{0}\right)$ for all $(z, \epsilon) \in$ $D(0, \delta / 2) \times\left(D\left(0, \epsilon_{0}\right) \backslash\{0\}\right)$ can be analytically continued on the domain $U_{d_{i+1}} \cup U_{d_{i}}$ (which is now a sector since $\left.U_{d_{i}} \cap U_{d_{i+1}} \neq \emptyset\right)$ by a function $V_{U_{d_{i+1}} \cup U_{d_{i}}, \mathcal{E}_{i} \cap \mathcal{E}_{i+1}}(\tau, z, \epsilon)$ on $\left(U_{d_{i+1}} \cup U_{d_{i}}\right) \times$ $D(0, \delta / 2) \times\left(\mathcal{E}_{i} \cap \mathcal{E}_{i+1}\right)$ which coincides with $V_{U_{d_{i}}, \mathcal{E}_{i}}(\tau, z, \epsilon)$ on $U_{d_{i}} \times D(0, \delta / 2) \times \mathcal{E}_{i} \cap \mathcal{E}_{i+1}$ and with $V_{U_{d_{i+1}}, \mathcal{E}_{i+1}}(\tau, z, \epsilon)$ on $U_{d_{i+1}} \times D(0, \delta / 2) \times\left(\mathcal{E}_{i} \cap \mathcal{E}_{i+1}\right)$ and which satisfies moreover the estimates

$$
\begin{aligned}
& \left|V_{U_{d_{i+1}} \cup U_{d_{i}}, \mathcal{E}_{i} \cap \mathcal{E}_{i+1}}(\tau, z, \epsilon)\right| \\
& \quad \leq C_{\Omega\left(d_{i}, d_{i+1}, \mathcal{E}_{i}, \mathcal{E}_{i+1}\right)}\left(1+\frac{|\tau|^{2}}{|\epsilon|^{2}}\right)^{-1} \exp \left(\frac{\sigma \zeta(b)}{|\epsilon|}|\tau|+\exp \left(\sigma^{\prime} \zeta(b)|\tau|\right)\right)
\end{aligned}
$$

for all $(\tau, z, \epsilon) \in\left(U_{d_{i+1}} \cup U_{d_{i}}\right) \times D(0, \delta / 2) \times\left(\mathcal{E}_{i} \cap \mathcal{E}_{i+1}\right)$ for some constant $C_{\Omega\left(d_{i}, d_{i+1}, \mathcal{E}_{i}, \mathcal{E}_{i+1}\right)}>0$.

Using (139), we can choose $\mu_{\mathcal{T}}>0$ large enough and some constants $0<C_{15}<C_{16}<\sigma^{\prime \prime} / \sigma^{\prime}$ with

$$
C_{15} \log \left|\frac{t}{\epsilon}\right| \leq\left|\log \left(\frac{t}{\epsilon} e^{\sqrt{-1} \theta_{i}}\right)\right| \leq C_{16} \log \left|\frac{t}{\epsilon}\right|, \quad C_{15} \log \left|\frac{t}{\epsilon}\right| \leq\left|\log \left(\frac{t}{\epsilon} e^{\sqrt{-1} \theta_{i+1}}\right)\right| \leq C_{16} \log \left|\frac{t}{\epsilon}\right|
$$

for all $t \in \mathcal{T} \cap\left\{|t|>\mu_{\mathcal{T}}\right\}$, all $\epsilon \in \mathcal{E}_{i} \cap \mathcal{E}_{i+1}$. The integral of

$$
\tau \mapsto V_{U_{d_{i+1}} \cup U_{d_{i}}, \mathcal{E}_{i} \cap \mathcal{E}_{i+1}}(\tau, z, \epsilon) \exp (-t \tau / \epsilon)
$$

along the segment

$$
\left[0, \frac{C_{15} \log \left|\frac{t}{\epsilon}\right|}{\sigma^{\prime \prime} \zeta(b)} e^{\sqrt{-1}\left(\gamma_{i+1}+\arg \left(\log \left(\frac{t}{\epsilon} e^{\sqrt{-1} \theta_{i+1}}\right)\right)\right)}\right]
$$

followed by an arc of circle with radius $\frac{C_{15} \log \left|\frac{t}{\epsilon}\right|}{\sigma^{\prime \prime} \zeta(b)}$ connecting

$$
\frac{C_{15} \log \left|\frac{t}{\epsilon}\right|}{\sigma^{\prime \prime} \zeta(b)} e^{\sqrt{-1}\left(\gamma_{i+1}+\arg \left(\log \left(\frac{t}{\epsilon} e^{\sqrt{-1} \theta_{i+1}}\right)\right)\right)} \text { and } \frac{C_{15} \log \left|\frac{t}{\epsilon}\right|}{\sigma^{\prime \prime} \zeta(b)} e^{\sqrt{-1}\left(\gamma_{i}+\arg \left(\log \left(\frac{t}{\epsilon} e^{\sqrt{-1} \theta_{i}}\right)\right)\right)}
$$

and along the segment

$$
\left[\frac{C_{15} \log \left|\frac{t}{\epsilon}\right|}{\sigma^{\prime \prime} \zeta(b)} e^{\sqrt{-1}\left(\gamma_{i}+\arg \left(\log \left(\frac{t}{\epsilon} e^{\sqrt{-1} \theta_{i}}\right)\right)\right)}, 0\right]
$$

is equal to zero. Therefore, using the representation (129) we can rewrite the difference $X_{U_{d_{i+1}}, \mathcal{E}_{i+1}}-$ $X_{U_{d_{i}}, \mathcal{E}_{i}}$ as a sum of three integrals,

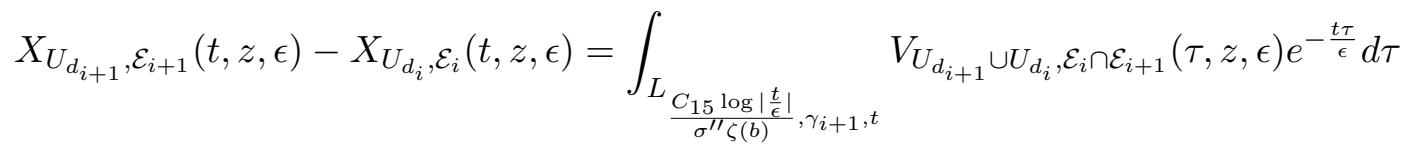

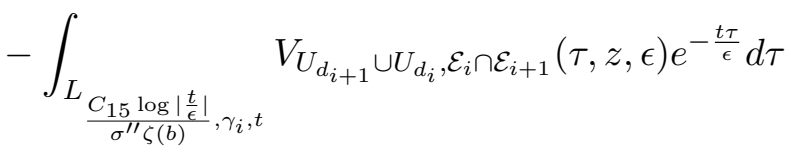

$$
\begin{aligned}
& +\int_{C\left(\frac{C_{15} \log \left|\frac{t}{\epsilon}\right|}{\sigma^{\prime \prime} \zeta(b)}, \gamma_{i}, \gamma_{i+1}, t\right)} V_{U_{d_{i+1}} \cup U_{d_{i}}, \mathcal{E}_{i} \cap \mathcal{E}_{i+1}}(\tau, z, \epsilon) e^{-\frac{t \tau}{\epsilon}} d \tau
\end{aligned}
$$

where

$$
\begin{aligned}
& L_{\frac{C_{15} \log \left|\frac{t}{\epsilon}\right|}{\sigma^{\prime \prime} \zeta(b)}, \gamma_{i}, t}=\left[\frac{C_{15} \log \left|\frac{t}{\epsilon}\right|}{\sigma^{\prime \prime} \zeta(b)} e^{\sqrt{-1}\left(\gamma_{i}+\arg \left(\log \left(\frac{t}{\epsilon} e^{\sqrt{-1} \theta_{i}}\right)\right)\right)}, e^{\sqrt{-1} \gamma_{i}} \frac{\log \left(\frac{t}{\epsilon} e^{\sqrt{-1} \theta_{i}}\right)}{\sigma^{\prime \prime} \zeta(b)}\right] \\
& L_{\frac{C_{15} \log \left|\frac{t}{\epsilon}\right|}{\sigma^{\prime \prime} \zeta(b)}, \gamma_{i+1}, t}=\left[\frac{C_{15} \log \left|\frac{t}{\epsilon}\right|}{\sigma^{\prime \prime} \zeta(b)} e^{\sqrt{-1}\left(\gamma_{i+1}+\arg \left(\log \left(\frac{t}{\epsilon} e^{\sqrt{-1} \theta_{i+1}}\right)\right)\right)}, e^{\sqrt{-1} \gamma_{i+1}} \frac{\log \left(\frac{t}{\epsilon} e^{\sqrt{-1} \theta_{i+1}}\right)}{\sigma^{\prime \prime} \zeta(b)}\right]
\end{aligned}
$$


and $C\left(\frac{C_{15} \log \left|\frac{t}{\epsilon}\right|}{\sigma^{\prime \prime} \zeta(b)}, \gamma_{i}, \gamma_{i+1}, t\right)$ is an arc of circle with radius $\frac{C_{15} \log \left|\frac{t}{\epsilon}\right|}{\sigma^{\prime \prime} \zeta(b)}$ connecting

$$
\frac{C_{15} \log \left|\frac{t}{\epsilon}\right|}{\sigma^{\prime \prime} \zeta(b)} e^{\sqrt{-1}\left(\gamma_{i}+\arg \left(\log \left(\frac{t}{\epsilon} e^{\sqrt{-1} \theta_{i}}\right)\right)\right)} \text { with } \frac{C_{15} \log \left|\frac{t}{\epsilon}\right|}{\sigma^{\prime \prime} \zeta(b)} e^{\sqrt{-1}\left(\gamma_{i+1}+\arg \left(\log \left(\frac{t}{\epsilon} e^{\sqrt{-1} \theta_{i+1}}\right)\right)\right)}
$$

with a well chosen orientation.

We provide estimates for the first integral

$$
I_{1}=\left|\int_{\frac{L_{\frac{C_{15} \log \left|\frac{t}{\epsilon}\right|}{\sigma^{\prime \prime} \zeta(b)}, \gamma_{i+1}, t}}{}} V_{U_{d_{i+1}} \cup U_{d_{i}}, \mathcal{E}_{i} \cap \mathcal{E}_{i+1}}(\tau, z, \epsilon) e^{-\frac{t \tau}{\epsilon}} d \tau\right|
$$

From the estimates (156) and (157) we deduce that

$$
\begin{aligned}
I_{1} \leq \int_{\frac{C_{15} \log \left|\frac{t}{\epsilon}\right|}{\sigma^{\prime \prime} \zeta(b)}}^{\frac{C_{16} \log \left|\frac{t}{\epsilon}\right|}{\sigma^{\prime \prime} \zeta(b)}} C_{\Omega\left(d_{i}, d_{i+1}, \mathcal{E}_{i}, \mathcal{E}_{i+1}\right)}\left(1+\frac{h^{2}}{|\epsilon|^{2}}\right)^{-1} \exp \left(\frac{\sigma \zeta(b)}{|\epsilon|} h+\exp \left(\sigma^{\prime} \zeta(b) h\right)\right) \\
\times \exp \left(-\operatorname{Re}\left(\frac{t}{\epsilon} h \exp \left(\sqrt{-1}\left(\gamma_{i+1}+\arg \left(\log \left(\frac{t}{\epsilon} e^{\sqrt{-1} \theta_{i+1}}\right)\right)\right)\right)\right)\right) d h
\end{aligned}
$$

From (141), (157) and (159), we deduce that

$$
\begin{aligned}
I_{1} \leq C_{\Omega\left(d_{i}, d_{i+1}, \mathcal{E}_{i}, \mathcal{E}_{i+1}\right)} \exp \left(\left|\frac{t}{\epsilon}\right|^{\frac{\sigma^{\prime} C_{16}}{\sigma^{\prime \prime}}}\right) \int_{\frac{C_{15} \log \left|\frac{t}{\epsilon}\right|}{\sigma^{\prime \prime} \zeta(b)}}^{\frac{C_{16} \log \left|\frac{t}{\epsilon}\right|}{\sigma^{\prime \prime}}} \exp \left(-\left(\delta_{1}^{\prime}|t|-\sigma \zeta(b)\right) \frac{h}{|\epsilon|}\right) d h \\
=C_{\Omega\left(d_{i}, d_{i+1}, \mathcal{E}_{i}, \mathcal{E}_{i+1}\right)} \exp \left(\left|\frac{t}{\epsilon}\right|^{\frac{\sigma^{\prime} C_{16}}{\sigma^{\prime \prime}}}\right) \frac{|\epsilon|}{\delta_{1}^{\prime}|t|-\sigma \zeta(b)} \\
\times\left(\exp \left(-\left(\delta_{1}^{\prime}|t|-\sigma \zeta(b)\right) \frac{C_{15} \log \left|\frac{t}{\epsilon}\right|}{\sigma^{\prime \prime} \zeta(b)|\epsilon|}\right)-\exp \left(-\left(\delta_{1}^{\prime}|t|-\sigma \zeta(b)\right) \frac{C_{16} \log \left|\frac{t}{\epsilon}\right|}{\sigma^{\prime \prime} \zeta(b)|\epsilon|}\right)\right) .
\end{aligned}
$$

From the choice of $C_{16}$ in (157), we can select a constant $0<\delta_{2}<\delta_{1}^{\prime} / 2$ such that

$$
|t|>\frac{\sigma \zeta(b)}{\delta_{1}^{\prime}-2 \delta_{2}} \quad, \quad\left(\frac{|t|}{\epsilon_{0}}\right)^{\frac{\sigma^{\prime} C_{16}}{\sigma^{\prime \prime}}-1} \leq \delta_{2} \log \left(\frac{\mu_{\mathcal{T}}}{\epsilon_{0}}\right) \frac{C_{15}}{\sigma^{\prime \prime} \zeta(b)}
$$

for all $t \in \mathcal{T} \cap\left\{|t|>\mu_{\mathcal{T}}\right\}$ (whenever $\mu_{\mathcal{T}}$ is large enough). Hence, we deduce that

$$
\begin{aligned}
\exp \left(\left|\frac{t}{\epsilon}\right| \frac{\sigma^{\prime} C_{16}}{\sigma^{\prime \prime}}\right) \exp \left(-\left(\delta_{1}^{\prime}|t|-\sigma \zeta(b)\right)\right. & \left.\frac{C_{15} \log \left|\frac{t}{\epsilon}\right|}{\sigma^{\prime \prime} \zeta(b)|\epsilon|}\right) \\
& \leq \exp \left(-\frac{\delta_{2} C_{15}}{\sigma^{\prime \prime} \zeta(b)} \frac{|t|}{|\epsilon|} \log \left|\frac{t}{\epsilon}\right|\right) \leq \exp \left(-\frac{\delta_{2} C_{15} \mu_{\mathcal{T}}}{\sigma^{\prime \prime} \zeta(b)} \frac{\log \left|\frac{\mu_{\mathcal{T}}}{\epsilon}\right|}{|\epsilon|}\right)
\end{aligned}
$$

for all $t \in \mathcal{T} \cap\left\{|t|>\mu_{\mathcal{T}}\right\}$, all $\epsilon \in \mathcal{E}_{i} \cap \mathcal{E}_{i+1}$. Taking into account (160) and (162), we deduce

$$
I_{1} \leq \frac{C_{\Omega\left(d_{i}, d_{i+1}, \mathcal{E}_{i}, \mathcal{E}_{i+1}\right)} \epsilon_{0}}{2 \delta_{2} \mu_{\mathcal{T}}} \exp \left(-\frac{\delta_{2} C_{15} \mu_{\mathcal{T}}}{\sigma^{\prime \prime} \zeta(b)} \frac{\log \left|\frac{\mu \mathcal{T}}{\epsilon}\right|}{|\epsilon|}\right)
$$

for all $t \in \mathcal{T} \cap\left\{|t|>\mu_{\mathcal{T}}\right\}$, all $\epsilon \in \mathcal{E}_{i} \cap \mathcal{E}_{i+1}$. 
Using similar estimates as above, one can also get the following estimates: there exists $\delta_{2}>0$ such that

$$
\begin{aligned}
& \left|\int_{\frac{L_{C_{15} \log \left|\frac{t}{\epsilon}\right|}}{\sigma^{\prime \prime} \zeta(b)}, \gamma_{i}, t} V_{U_{d_{i+1}} \cup U_{d_{i}}, \mathcal{E}_{i} \cap \mathcal{E}_{i+1}}(\tau, z, \epsilon) e^{-\frac{t \tau}{\epsilon}} d \tau\right| \\
& \leq \frac{C_{\Omega\left(d_{i}, d_{i+1}, \mathcal{E}_{i}, \mathcal{E}_{i+1}\right)} \epsilon_{0}}{2 \delta_{2} \mu_{\mathcal{T}}} \exp \left(-\frac{\delta_{2} C_{15} \mu_{\mathcal{T}}}{\sigma^{\prime \prime} \zeta(b)} \frac{\log \left|\frac{\mu \mathcal{T}}{\epsilon}\right|}{|\epsilon|}\right)
\end{aligned}
$$

for all $t \in \mathcal{T} \cap\left\{|t|>\mu_{\mathcal{T}}\right\}$, all $\epsilon \in \mathcal{E}_{i} \cap \mathcal{E}_{i+1}$.

In the last part, we consider the third integral

$$
I_{3}=\left|\int_{C\left(\frac{C_{15} \log \left|\frac{t}{\epsilon}\right|}{\sigma^{\prime \prime} \zeta(b)}, \gamma_{i}, \gamma_{i+1}, t\right)} V_{U_{d_{i+1}} \cup U_{d_{i}}, \mathcal{E}_{i} \cap \mathcal{E}_{i+1}}(\tau, z, \epsilon) e^{-\frac{t \tau}{\epsilon}} d \tau\right| .
$$

From (156), we deduce that

$$
\begin{aligned}
& I_{3} \leq \mid \int_{\gamma_{i}+\arg \left(\log \left(\frac{t}{\epsilon} e^{\sqrt{-1}} \theta_{i}\right)\right)}^{\gamma_{i+1}+\arg \left(\log \left(\frac{t}{\epsilon} e^{\sqrt{-1} \theta_{i+1}}\right)\right)} \times C_{\Omega\left(d_{i}, d_{i+1}, \mathcal{E}_{i}, \mathcal{E}_{i+1}\right)} \\
& \times \exp \left(\frac{\sigma C_{15} \log \left|\frac{t}{\epsilon}\right|}{|\epsilon| \sigma^{\prime \prime}}+\right.\left.\exp \left(\sigma^{\prime} \frac{C_{15} \log \left|\frac{t}{\epsilon}\right|}{\sigma^{\prime \prime}}\right)\right) \\
& \times e^{-\frac{|t| C_{15} \log \left|\frac{t}{\epsilon}\right|}{|\epsilon| \sigma^{\prime \prime} \zeta(b)} \cos (\theta+\arg (t)-\arg (\epsilon))} \frac{C_{15} \log \left|\frac{t}{\epsilon}\right|}{\sigma^{\prime \prime} \zeta(b)} d \theta \mid
\end{aligned}
$$

Using (139), the arc of circle $C\left(\frac{C_{15} \log \left|\frac{t}{\epsilon}\right|}{\sigma^{\prime \prime} \zeta(b)}, \gamma_{i}, \gamma_{i+1}, t\right)$ can be chosen in such a way that $\cos (\theta+$ $\arg (t)-\arg (\epsilon)) \geq \delta_{1}^{\prime}$, for all

$$
\begin{gathered}
\theta \in\left[\gamma_{i}+\arg \left(\log \left(\frac{t}{\epsilon} e^{\sqrt{-1} \theta_{i}}\right)\right), \gamma_{i+1}+\arg \left(\log \left(\frac{t}{\epsilon} e^{\sqrt{-1} \theta_{i+1}}\right)\right)\right] \text { if } \gamma_{i}<\gamma_{i+1}, \\
\theta \in\left[\gamma_{i+1}+\arg \left(\log \left(\frac{t}{\epsilon} e^{\sqrt{-1} \theta_{i+1}}\right)\right), \gamma_{i}+\arg \left(\log \left(\frac{t}{\epsilon} e^{\sqrt{-1} \theta_{i}}\right)\right)\right] \text { if } \gamma_{i+1}<\gamma_{i},
\end{gathered}
$$

for all $t \in \mathcal{T} \cap\left\{|t|>\mu_{\mathcal{T}}\right\}$, all $\epsilon \in \mathcal{E}_{i} \cap \mathcal{E}_{i+1}$. From (165) and using (139) we deduce that

$$
\begin{aligned}
& I_{3} \leq\left(\left|\gamma_{i+1}-\gamma_{i}\right|+\pi\right) C_{\Omega\left(d_{i}, d_{i+1}, \mathcal{E}_{i}, \mathcal{E}_{i+1}\right)} \exp \left(\left|\frac{t}{\epsilon}\right|^{\frac{\sigma^{\prime} C_{15}}{\sigma^{\prime \prime}}}\right) \frac{C_{15} \log \left|\frac{t}{\epsilon}\right|}{\sigma^{\prime \prime} \zeta(b)} \\
& \times \exp \left(-\left(\delta_{1}^{\prime}|t|-\sigma \zeta(b)\right) \frac{C_{15} \log \left|\frac{t}{\epsilon}\right|}{\sigma^{\prime \prime} \zeta(b)|\epsilon|}\right)
\end{aligned}
$$

for all $t \in \mathcal{T} \cap\left\{|t|>\mu_{\mathcal{T}}\right\}$ and for all $\epsilon \in \mathcal{E}_{i+1} \cap \mathcal{E}_{i}$.

From the choice of $C_{15}$ in (157), we can select a constant $0<\delta_{2}<\delta_{1}^{\prime} / 3$ such that

$$
|t| \geq \frac{\sigma \zeta(b)}{\delta_{1}^{\prime}-3 \delta_{2}} \quad, \quad\left(\frac{|t|}{\epsilon_{0}}\right)^{\frac{\sigma^{\prime} C_{15}}{\sigma^{\prime \prime}}-1} \leq \delta_{2} \log \left(\frac{\mu_{\mathcal{T}}}{\epsilon_{0}}\right) \frac{C_{15}}{\sigma^{\prime \prime} \zeta(b)}
$$

for all $t \in \mathcal{T} \cap\left\{|t|>\mu_{\mathcal{T}}\right\}$ (whenever $\mu_{\mathcal{T}}$ is large enough). Therefore, we get that

$$
\exp \left(\left|\frac{t}{\epsilon}\right| \frac{\sigma^{\prime} C_{15}}{\sigma^{\prime \prime}}\right) \exp \left(-\frac{\delta_{2} C_{15}}{\sigma^{\prime \prime} \zeta(b)}\left|\frac{t}{\epsilon}\right| \log \left|\frac{t}{\epsilon}\right|\right) \leq \exp \left(\left|\frac{t}{\epsilon}\right|^{\frac{\sigma^{\prime} C_{15}}{\sigma^{\prime \prime}}}\right) \exp \left(-\frac{\delta_{2} C_{15}}{\sigma^{\prime \prime} \zeta(b)}\left|\frac{t}{\epsilon}\right| \log \left(\frac{\mu_{\mathcal{T}}}{\epsilon_{0}}\right)\right) \leq 1
$$


for all $t \in \mathcal{T} \cap\left\{|t|>\mu_{\mathcal{T}}\right\}$ and for all $\epsilon \in \mathcal{E}_{i+1} \cap \mathcal{E}_{i}$. Moreover, from (25), we deduce that

$$
\begin{aligned}
\frac{C_{15} \log \left|\frac{t}{\epsilon}\right|}{\sigma^{\prime \prime} \zeta(b)} \exp \left(-\frac{\delta_{2} C_{15}}{\sigma^{\prime \prime} \zeta(b)}\left|\frac{t}{\epsilon}\right| \log \left|\frac{t}{\epsilon}\right|\right) \leq \frac{C_{15} \log \left|\frac{t}{\epsilon}\right|}{\sigma^{\prime \prime} \zeta(b)} \exp \left(-\frac{\delta_{2} C_{15}}{\sigma^{\prime \prime} \zeta(b)} \frac{\mu_{\mathcal{T}}}{\epsilon_{0}} \log \left|\frac{t}{\epsilon}\right|\right) \\
\leq \frac{C_{15}}{\sigma^{\prime \prime} \zeta(b)} \sup _{x \geq 0} x \exp \left(-\frac{\delta_{2} C_{15} \mu_{\mathcal{T}}}{\sigma^{\prime \prime} \zeta(b) \epsilon_{0}} x\right)=\frac{\epsilon_{0} e^{-1}}{\delta_{2} \mu_{\mathcal{T}}}
\end{aligned}
$$

for all $t \in \mathcal{T} \cap\left\{|t|>\mu_{\mathcal{T}}\right\}$ and for all $\epsilon \in \mathcal{E}_{i+1} \cap \mathcal{E}_{i}$. From (166), (168), (169), we get that

$$
\begin{aligned}
I_{3} \leq\left(\left|\gamma_{i+1}-\gamma_{i}\right|+\right. & \pi) C_{\Omega\left(d_{i}, d_{i+1}, \mathcal{E}_{i}, \mathcal{E}_{i+1}\right)} \frac{\epsilon_{0} e^{-1}}{\delta_{2} \mu_{\mathcal{T}}} \exp \left(-\frac{\delta_{2} C_{15}}{\sigma^{\prime \prime} \zeta(b)}\left|\frac{t}{\epsilon}\right| \log \left|\frac{t}{\epsilon}\right|\right) \\
& \leq\left(\left|\gamma_{i+1}-\gamma_{i}\right|+\pi\right) C_{\Omega\left(d_{i}, d_{i+1}, \mathcal{E}_{i}, \mathcal{E}_{i+1}\right)} \frac{\epsilon_{0} e^{-1}}{\delta_{2} \mu_{\mathcal{T}}} \exp \left(-\frac{\delta_{2} C_{15}}{\sigma^{\prime \prime} \zeta(b)} \frac{\mu_{\mathcal{T}}}{|\epsilon|} \log \left|\frac{\mu_{\mathcal{T}}}{\epsilon}\right|\right)
\end{aligned}
$$

for all $t \in \mathcal{T} \cap\left\{|t|>\mu_{\mathcal{T}}\right\}$ and for all $\epsilon \in \mathcal{E}_{i+1} \cap \mathcal{E}_{i}$.

Finally, gathering (158), (163), (164) and (170), we deduce that the estimates (155) hold.

\section{Existence of formal series solutions in the complex parameter for the singularly perturbed problem and asymptotic expan- sions with two levels}

\subsection{A Ramis-Sibuya theorem with two levels}

Definition 4 Let $\left(\mathbb{E},\|.\|_{\mathbb{E}}\right)$ be a Banach space over $\mathbb{C}$. We denote $\mathbb{E}[[\epsilon]]$ the space of all formal series $\sum_{k \geq 0} a_{k} \epsilon^{k}$ with coefficients $a_{k}$ in $\mathbb{E}$ for all integers $k \geq 0$. Let $f: \mathcal{E} \rightarrow \mathbb{E}$ be a holomorphic function on a bounded open sector $\mathcal{E}$ centered at 0 . Let $\hat{f}(\epsilon)=\sum_{k \geq 0} a_{k} \epsilon^{k} \in \mathbb{E}[[\epsilon]]$ be a formal series.

1) We say that $f$ has the formal series $\hat{f}$ as $1-$ Gevrey asymptotic expansion if, for any closed proper subsector $\mathcal{W} \subset \mathcal{E}$ centered at 0 , there exist $C, M>0$ such that

$$
\left\|f(\epsilon)-\sum_{k=0}^{N-1} a_{k} \epsilon^{k}\right\|_{\mathbb{E}} \leq C M^{N}(N / e)^{N}|\epsilon|^{N}
$$

for all $N \geq 1$, all $\epsilon \in \mathcal{W}$.

2) We say that $f$ has the formal series $\hat{f}$ as $1^{+}$- Gevrey asymptotic expansion if, for any closed proper subsector $\mathcal{W} \subset \mathcal{E}$ centered at 0 , there exist $C, M>0$ such that

$$
\left\|f(\epsilon)-\sum_{k=0}^{N-1} a_{k} \epsilon^{k}\right\|_{\mathbb{E}} \leq C M^{N}(N / \log N)^{N}|\epsilon|^{N}
$$

for all $N \geq 2$, all $\epsilon \in \mathcal{W}$. In particular, the formal series $\hat{f}$ is itself of $1^{+}-$Gevrey type, meaning that there exist two constants $C^{\prime}, M^{\prime}>0$ such that $\left\|a_{k}\right\|_{\mathbb{E}} \leq C^{\prime} M^{\prime k}(k / \log k)^{k}$ for all $k \geq 2$. 
In this section, we give a new version of the classical Ramis-Sibuya theorem (see [17], Theorem XI-2-3) that involves two kinds of Gevrey levels, the $1-$ Gevrey order and the $1^{+}-$Gevrey order.

Theorem (RS) Let $\left(\mathbb{E},\|.\|_{\mathbb{E}}\right)$ be a Banach space over $\mathbb{C}$ and $\left\{\mathcal{E}_{i}\right\}_{0 \leq i \leq \nu-1}$ be a good covering in $\mathbb{C}^{*}$, see Definition 3. For all $0 \leq i \leq \nu-1$, let $G_{i}$ be a holomorphic function from $\mathcal{E}_{i}$ into the Banach space $\left(\mathbb{E},\|.\|_{\mathbb{E}}\right)$ and let the cocycle $\Delta_{i}(\epsilon)=G_{i+1}(\epsilon)-G_{i}(\epsilon)$ be a holomorphic function from the sector $Z_{i}=\mathcal{E}_{i+1} \cap \mathcal{E}_{i}$ into $\mathbb{E}$ (with the convention that $\mathcal{E}_{\nu}=\mathcal{E}_{0}$ and $G_{\nu}=G_{0}$ ). We make the following assumptions.

1) The functions $G_{i}(\epsilon)$ are bounded as $\epsilon \in \mathcal{E}_{i}$ tends to the origin in $\mathbb{C}$, for all $0 \leq i \leq \nu-1$.

2) For some finite subset $I_{1} \subset\{0, \ldots \nu-1\}$ and for all $i \in I_{1}$, the functions $\Delta_{i}(\epsilon)$ are exponentially flat on $Z_{i}$, for all $0 \leq i \leq \nu-1$. This means that there exist constants $K_{i}, M_{i}>0$ such that

$$
\left\|\Delta_{i}(\epsilon)\right\|_{\mathbb{E}} \leq K_{i} e^{-M_{i} /|\epsilon|}
$$

for all $\epsilon \in Z_{i}$.

3) For all $i \in I_{2}=\{0, \ldots, \nu-1\} \backslash I_{1}$, the functions $\Delta_{i}(\epsilon)$ are super-exponentially flat on $Z_{i}$, for all $0 \leq i \leq \nu-1$. This means that there exist constants $K_{i}, M_{i}>0$ and $L_{i}>1$ such that

$$
\|\left.\Delta_{i}(\epsilon)\right|_{\mathbb{E}} \leq K_{i} \exp \left(-\frac{M_{i}}{|\epsilon|} \log \frac{L_{i}}{|\epsilon|}\right)
$$

for all $\epsilon \in Z_{i}$.

Then, there exist a convergent power series $a(\epsilon) \in \mathbb{E}\{\epsilon\}$ near $\epsilon=0$ and two formal series $\hat{G}^{1}(\epsilon), \hat{G}^{2}(\epsilon) \in \mathbb{E}[[\epsilon]]$ such that $G_{i}(\epsilon)$ owns the following decomposition

$$
G_{i}(\epsilon)=a(\epsilon)+G_{i}^{1}(\epsilon)+G_{i}^{2}(\epsilon)
$$

where $G_{i}^{1}(\epsilon)$ is holomorphic on $\mathcal{E}_{i}$ and has $\hat{G}^{1}(\epsilon)$ as 1 -Gevrey asymptotic expansion on $\mathcal{E}_{i}$, $G_{i}^{2}(\epsilon)$ is holomorphic on $\mathcal{E}_{i}$ and carries $\hat{G}^{2}(\epsilon)$ as $1^{+}$-Gevrey asymptotic expansion on $\mathcal{E}_{i}$, for all $0 \leq i \leq \nu-1$.

Proof We consider two holomorphic cocycles $\Delta_{i}^{1}(\epsilon)$ and $\Delta_{i}^{2}(\epsilon)$ defined on the sectors $Z_{i}$ in the following way:

$$
\Delta_{i}^{1}(\epsilon)=\left\{\begin{array}{ll}
\Delta_{i}(\epsilon) & \text { if } i \in I_{1} \\
0 & \text { if } i \in I_{2}
\end{array} \quad, \quad \Delta_{i}^{2}(\epsilon)= \begin{cases}0 & \text { if } i \in I_{1} \\
\Delta_{i}(\epsilon) & \text { if } i \in I_{2}\end{cases}\right.
$$

for all $\epsilon \in Z_{i}$, all $0 \leq i \leq \nu-1$. We will need the following two lemma.

Lemma 14 For all $0 \leq i \leq \nu-1$, there exist bounded holomorphic functions $\Psi_{i}^{1}: \mathcal{E}_{i} \rightarrow \mathbb{C}$ such that

$$
\Delta_{i}^{1}(\epsilon)=\Psi_{i+1}^{1}(\epsilon)-\Psi_{i}^{1}(\epsilon)
$$

for all $\epsilon \in Z_{i}$, where by convention $\Psi_{\nu}^{1}(\epsilon)=\Psi_{0}^{1}(\epsilon)$. Moreover, there exist coefficients $\varphi_{m}^{1} \in \mathbb{E}$, $m \geq 0$, such that for each $0 \leq l \leq \nu-1$ and any closed proper subsector $\mathcal{W} \subset \mathcal{E}_{l}$, centered at 0 , there exist two constants $\breve{K}_{l}, \breve{M}_{l}>0$ with

$$
\left\|\Psi_{l}^{1}(\epsilon)-\sum_{m=0}^{M-1} \varphi_{m}^{1} \epsilon^{m}\right\|_{\mathbb{E}} \leq \breve{K}_{l}\left(\breve{M}_{l}\right)^{M}\left(\frac{M}{e}\right)^{M}|\epsilon|^{M}
$$

for all $\epsilon \in \mathcal{W}$, all $M \geq 1$. 
Proof The proof is a consequence of Lemma XI-2-6 from [17] which provides the so-called classical Ramis-Sibuya theorem in Gevrey classes.

Lemma 15 For all $0 \leq i \leq \nu-1$, there exist bounded holomorphic functions $\Psi_{i}^{2}: \mathcal{E}_{i} \rightarrow \mathbb{C}$ such that

$$
\Delta_{i}^{2}(\epsilon)=\Psi_{i+1}^{2}(\epsilon)-\Psi_{i}^{2}(\epsilon)
$$

for all $\epsilon \in Z_{i}$, where by convention $\Psi_{\nu}^{2}(\epsilon)=\Psi_{0}^{2}(\epsilon)$. Moreover, there exist coefficients $\varphi_{m}^{2} \in \mathbb{E}$, $m \geq 0$, such that for each $0 \leq l \leq \nu-1$ and any closed proper subsector $\mathcal{W} \subset \mathcal{E}_{l}$, centered at 0 , there exist two constants $\hat{K}_{l}, \hat{M}_{l}>0$ with

$$
\left\|\Psi_{l}^{2}(\epsilon)-\sum_{m=0}^{M-1} \varphi_{m}^{2} \epsilon^{m}\right\|_{\mathbb{E}} \leq \hat{K}_{l}\left(\hat{M}_{l}\right)^{M}\left(\frac{M}{\log M}\right)^{M}|\epsilon|^{M}
$$

for all $\epsilon \in \mathcal{W}$, all $M \geq 2$.

Proof We will follow the same arguments as in Lemma XI-2-6 from [17] with appropriate modifications in the asymptotic expansions of the functions constructed with the help of the Cauchy-Heine transform.

For all $0 \leq l \leq \nu-1$, we choose a segment

$$
\mathcal{C}_{l}=\left\{t e^{\sqrt{-1} \theta_{l}}, t \in[0, r]\right\} \subset \mathcal{E}_{l} \cap \mathcal{E}_{l+1} .
$$

These $\nu$ segments divide the open punctured disc $D(0, r) \backslash\{0\}$ into $\nu$ open sectors $\tilde{\mathcal{E}}_{0}, \ldots, \tilde{\mathcal{E}}_{\nu-1}$ where

$$
\tilde{\mathcal{E}}_{l}=\left\{\epsilon \in \mathbb{C}^{*} / \theta_{l-1}<\arg (\epsilon)<\theta_{l},|\epsilon|<r\right\} \quad, \quad 0 \leq l \leq \nu-1,
$$

where by convention $\theta_{-1}=\theta_{\nu-1}$. Let

$$
\Psi_{l}^{2}(\epsilon)=\frac{-1}{2 \pi \sqrt{-1}} \sum_{h=0}^{\nu-1} \int_{C_{h}} \frac{\Delta_{h}^{2}(\xi)}{\xi-\epsilon} d \xi
$$

for all $\epsilon \in \tilde{\mathcal{E}}_{l}$, for $0 \leq l \leq \nu-1$, be defined as a sum of Cauchy-Heine transforms of the functions $\Delta_{h}^{2}(\epsilon)$. By deformation of the paths $C_{l-1}$ and $C_{l}$ without moving their endpoints and letting the other paths $C_{h}, h \neq l-1, l$ untouched (with the convention that $C_{-1}=C_{\nu-1}$ ), one can continue analytically the function $\Psi_{l}^{2}$ onto $\mathcal{E}_{l}$. Therefore, $\Psi_{l}^{2}$ defines a holomorphic function on $\mathcal{E}_{l}$, for all $0 \leq l \leq \nu-1$.

Now, take $\epsilon \in \mathcal{E}_{l} \cap \mathcal{E}_{l+1}$. In order to compute $\Psi_{l+1}^{2}(\epsilon)-\Psi_{l}^{2}(\epsilon)$, we write

$$
\begin{aligned}
\Psi_{l}^{2}(\epsilon)=\frac{-1}{2 \pi \sqrt{-1}} \int_{\hat{C}_{l}} \frac{\Delta_{l}^{2}(\xi)}{\xi-\epsilon} d \xi+\frac{-1}{2 \pi \sqrt{-1}} \sum_{h=0, h \neq l}^{\nu-1} \int_{C_{h}} \frac{\Delta_{h}^{2}(\xi)}{\xi-\epsilon} d \xi \\
\Psi_{l+1}^{2}(\epsilon)=\frac{-1}{2 \pi \sqrt{-1}} \int_{\check{C}_{l}} \frac{\Delta_{l}^{2}(\xi)}{\xi-\epsilon} d \xi+\frac{-1}{2 \pi \sqrt{-1}} \sum_{h=0, h \neq l}^{\nu-1} \int_{C_{h}} \frac{\Delta_{h}^{2}(\xi)}{\xi-\epsilon} d \xi
\end{aligned}
$$

where the paths $\hat{C}_{l}$ and $\check{C}_{l}$ are obtained by deforming the same path $C_{l}$ without moving its endpoints in such a way that:

(a) $\hat{C}_{l} \subset \mathcal{E}_{l} \cap \mathcal{E}_{l+1}$ and $\check{C}_{l} \subset \mathcal{E}_{l} \cap \mathcal{E}_{l+1}$, 
(b) $\Gamma_{l, l+1}:=-\check{C}_{l}+\hat{C}_{l}$ is a simple closed curve with positive orientation whose interior contains $\epsilon$.

Therefore, due to the residue formula, we can write

$$
\Psi_{l+1}^{2}(\epsilon)-\Psi_{l}^{2}(\epsilon)=\frac{1}{2 \pi \sqrt{-1}} \int_{\Gamma_{l, l+1}} \frac{\Delta_{l}^{2}(\xi)}{\xi-\epsilon} d \xi=\Delta_{l}^{2}(\epsilon)
$$

for all $\epsilon \in \mathcal{E}_{l} \cap \mathcal{E}_{l+1}$, for all $0 \leq l \leq \nu-1$ (with the convention that $\Psi_{\nu}^{2}=\Psi_{0}^{2}$ ).

In a second step, we derive asymptotic properties of $\Psi_{l}^{2}$. We fix an $0 \leq l \leq \nu-1$ and a proper closed sector $\mathcal{W}$ contained in $\mathcal{E}_{l}$. Let $\tilde{C}_{l}$ (resp. $\tilde{C}_{l-1}$ ) be a path obtained by deforming $C_{l}$ (resp. $C_{l-1}$ ) without moving the endpoints in order that $\mathcal{W}$ is contained in the interior of the simple closed curve $\tilde{C}_{l-1}+\gamma_{l}-\tilde{C}_{l}$ (which is itself contained in $\mathcal{E}_{l}$ ), where $\gamma_{l}$ is a circular arc joining the two points $r e^{\sqrt{-1} \theta_{l-1}}$ and $r e^{\sqrt{-1} \theta_{l}}$. We get the representation

$$
\begin{aligned}
\Psi_{l}^{2}(\epsilon)=\frac{-1}{2 \pi \sqrt{-1}} \int_{\tilde{C}_{l}} \frac{\Delta_{l}^{2}(\xi)}{\xi-\epsilon} d \xi+\frac{-1}{2 \pi \sqrt{-1}} \int_{\tilde{C}_{l-1}} \frac{\Delta_{l-1}^{2}(\xi)}{\xi-\epsilon} d \xi & \\
& +\frac{-1}{2 \pi \sqrt{-1}} \sum_{h=0, h \neq l, l-1}^{\nu-1} \int_{C_{h}} \frac{\Delta_{h}^{2}(\xi)}{\xi-\epsilon} d \xi
\end{aligned}
$$

for all $\epsilon \in \mathcal{W}$. One assumes that the path $\tilde{C}_{l}$ is given as the union of a segment $L_{l}=\left\{t e^{\sqrt{-1} w_{l}} / t \in\right.$ $\left.\left[0, r_{1}\right]\right\}$ where $r_{1}<r$ and $w_{l}>\theta_{l}$ and a curve $\Gamma_{l}=\left\{\mu_{l}(\tau) / \tau \in[0,1]\right\}$ such that $\mu_{l}(0)=r_{1} e^{\sqrt{-1} w_{l}}$, $\mu_{l}(1)=r e^{\sqrt{-1} \theta_{l}}$ and $r_{1} \leq\left|\mu_{l}(\tau)\right|<r$ for all $\tau \in[0,1)$. We also assume that there exists a positive number $\sigma<1$ with $|\epsilon| \leq \sigma r_{1}$ for all $\epsilon \in \mathcal{W}$. By construction of the path $\Gamma_{l}$, we get that the function $\epsilon \mapsto \frac{1}{2 \pi \sqrt{-1}} \int_{\Gamma_{l}} \frac{\Delta_{l}^{2}(\xi)}{\xi-\epsilon} d \xi$ defines an analytic function on the open disc $D\left(0, r_{1}\right)$.

It remains to give estimates for the integral $\frac{1}{2 \pi \sqrt{-1}} \int_{L_{l}} \frac{\Delta_{l}^{2}(\xi)}{\xi-\epsilon} d \xi$. Let $M \geq 0$ be an integer. From the usual geometric series expansion, one can write

$$
\frac{1}{2 \pi \sqrt{-1}} \int_{L_{l}} \frac{\Delta_{l}^{2}(\xi)}{\xi-\epsilon} d \xi=\sum_{m=0}^{M} \alpha_{l, m}^{2} \epsilon^{m}+\epsilon^{M+1} E_{l, M+1}(\epsilon)
$$

where

$$
\alpha_{l, m}^{2}=\frac{1}{2 \pi \sqrt{-1}} \int_{L_{l}} \frac{\Delta_{l}^{2}(\xi)}{\xi^{m+1}} d \xi \quad, \quad E_{l, M+1}(\epsilon)=\frac{1}{2 \pi \sqrt{-1}} \int_{L_{l}} \frac{\Delta_{l}^{2}(\xi)}{\xi^{M+1}(\xi-\epsilon)} d \xi
$$

for all $\epsilon \in \mathcal{W}$.

Gathering (174) and (183), we get some constants $K_{l}, M_{l}>0$ and $L_{l}>1$ such that

$$
\begin{aligned}
\left\|\alpha_{l, m}^{2}\right\|_{\mathbb{E}} \leq \frac{K_{l}}{2 \pi} \int_{0}^{r_{1}} \frac{\exp \left(-\frac{M_{l}}{\tau} \log \frac{L_{l}}{\tau}\right)}{\tau^{m+1}} d \tau & \leq \frac{K_{l}}{2 \pi} \int_{0}^{+\infty} \frac{\exp \left(-\frac{M_{l}}{\tau} \log \frac{L_{l}}{\tau}\right)}{\tau^{m+1}} d \tau \\
& =\frac{K_{l}}{2 \pi} \int_{0}^{+\infty} u^{m-1} \exp \left(-M_{l} u \log \left(L_{l} u\right)\right) d u=A_{l, m}
\end{aligned}
$$

Lemma 16 There exist two constants $\check{K}_{l}, \check{M}_{l}>0$ such that

$$
A_{l, m} \leq \check{K}_{l}\left(\check{M}_{l}\right)^{m}\left(\frac{m}{\log m}\right)^{m}
$$

for all $m \geq 2$. 
Proof Since $L_{l}>1$, one gets that $A_{l, m} \leq \frac{K_{l}}{2 \pi} B_{l, m}$ where

$$
B_{l, m}=\int_{0}^{+\infty} u^{m-1} \exp \left(-M_{l} u \log (u)\right) d u
$$

for all $m \geq 1$. We consider now the function

$$
L(t)=\int_{0}^{+\infty} \exp \left(-M_{l} u \log (u)\right) \exp (t u) d u
$$

which defines an entire function on $\mathbb{C}$. Now, we follow the example 2.6 p. 125 of [11] (see also Example 7.5 p. 83 from [29]), in order to give the leading term behaviour of $L(t)$ as $t$ tends to infinity using the Laplace method. For all $t \in \mathbb{C}$, we have that

$$
|L(t)| \leq I(|t|)=\int_{0}^{+\infty} \exp \left(-M_{l} u \log (u)\right) \exp (|t| u) d u
$$

The function $f(u)=|t| u-M_{l} u \log (u)$ reaches his unique maximum at $u=\exp \left(\frac{|t|}{M_{l}}-1\right)=\xi$. Now, we make the change of variable $u=\xi s$ in the integral $I(|t|)$ which yields

$$
I(|t|)=\xi \int_{0}^{+\infty} e^{\xi M_{l}(s-s \log (s))} d s .
$$

Now, the function $h(s)=M_{l}(s-s \log s)$ takes its unique maximum value at $s=1$, with $h(1)=M_{l}$ and $h^{\prime \prime}(1)=-M_{l}$. From Proposition 2.5 p. 125 in [11] (Laplace method), we get that

$$
\xi \int_{0}^{+\infty} e^{\xi M_{l}(s-s \log (s))} d s \sim\left(\frac{2 \pi}{M_{l}}\right)^{1 / 2} \xi^{1 / 2} e^{\xi M_{l}}
$$

as $\xi$ tends to $+\infty$ (where $f(\xi) \sim g(\xi)$ means that $f$ and $g$ are equivalent as $\xi \rightarrow+\infty$ ). Therefore, we get a constant $\stackrel{\circ}{K}$ l $>0$ such that

$$
|L(t)| \leq \stackrel{\circ}{K_{l}} e^{\frac{1}{2}\left(\frac{|t|}{M_{l}}-1\right)} \exp \left(M_{l} e^{\frac{|t|}{M_{l}}-1}\right)
$$

for all $t \in \mathbb{C}$, which means that $L(t)$ has at most super-exponential growth at infinity.

From the Cauchy formula, for all $k \geq 2$, one can write the derivative of order $k$ of $L$ at 0 in the form

$$
L^{(k)}(0)=\frac{k !}{2 \sqrt{-1} \pi} \int_{C(0, R)} \frac{L(\xi)}{\xi^{k+1}} d \xi
$$

for any circle $C(0, R)$ centered at 0 with radius $R>0$. Now, take the particular radius $R=$ $M_{l} \log k$. From (187) and the Stirling formula, we get two constants $\check{K}_{l}, \check{M}_{l}>0$ such that

$$
\left|L^{(k)}(0)\right| \leq \check{K}_{l}\left(\check{M}_{l}\right)^{k}\left(\frac{k}{\log k}\right)^{k}
$$

for all $k \geq 2$. On the other hand, by derivation under the sign $\int$, one also gets an other expression for the Taylor coefficients of $L$ at 0 ,

$$
L^{(k)}(0)=B_{l, k+1}
$$

for all $k \geq 0$. Gathering (188) and (189) yields the lemma. 
Moreover, as above, one can choose a positive number $\eta>0$ (depending on $\mathcal{W}$ ) such that $|\xi-\epsilon| \geq|\xi| \sin (\eta)$ for all $\xi \in L_{l}$ and all $\epsilon \in \mathcal{W}$. Again by (174), (183) and Lemma 16 we obtain two constants $\tilde{K}_{l}, \tilde{M}_{l}>0$ with

$$
\left\|E_{l, M+1}(\epsilon)\right\|_{\mathbb{E}} \leq \frac{K_{l}}{2 \pi \sin (\eta)} \int_{0}^{r_{1}} \frac{\exp \left(-\frac{M_{l}}{\tau} \log \frac{L_{l}}{\tau}\right)}{\tau^{M+2}} d \tau \leq \tilde{K}_{l}\left(\tilde{M}_{l}\right)^{M+1}\left(\frac{M+1}{\log (M+1)}\right)^{M+1}
$$

for all $\epsilon \in \mathcal{W}$.

Using comparable arguments, one can give estimates of the form (182), (184), (185) and (190) for the other integrals

$$
\frac{-1}{2 \pi \sqrt{-1}} \int_{\tilde{C}_{l-1}} \frac{\Delta_{l-1}^{2}(\xi)}{\xi-\epsilon} d \xi, \frac{-1}{2 \pi \sqrt{-1}} \int_{C_{h}} \frac{\Delta_{h}^{2}(\xi)}{\xi-\epsilon} d \xi
$$

for all $h \neq l, l-1$.

As a consequence, for any $0 \leq l \leq \nu-1$, there exist coefficients $\varphi_{l, m}^{2} \in \mathbb{E}, m \geq 0$ and two constants $\hat{K}_{l}, \hat{M}_{l}>0$ such that

$$
\left\|\Psi_{l}^{2}(\epsilon)-\sum_{m=0}^{M-1} \varphi_{l, m}^{2} \epsilon^{m}\right\|_{\mathbb{E}} \leq \hat{K}_{l}\left(\hat{M}_{l}\right)^{M}(M / \log M)^{M}|\epsilon|^{M}
$$

for all $M \geq 2$, all $\epsilon \in \mathcal{W}$.

From (174) and (180), we have in particular that there exist two constants $\hat{K}_{l}, \hat{M}_{l}>0$ with

$$
\| \Psi_{l+1}^{2}(\epsilon)-\left.\Psi_{l}^{2}(\epsilon)\right|_{\mathbb{E}} \leq \hat{K}_{l} e^{-\hat{M}_{l} /|\epsilon|}
$$

for all $\epsilon \in \mathcal{E}_{l} \cap \mathcal{E}_{l+1}$ and all $0 \leq l \leq \nu-1$. From Theorem XI-3-2 in [17], we deduce that $\Psi_{l+1}^{2}(\epsilon)-\Psi_{l}^{2}(\epsilon)$ has the formal series $\hat{0}$ as $1-$ Gevrey asymptotic expansion. From the unicity of the asymptotic expansions on sectors, we deduce that all the formal series $\sum_{m \geq 0} \varphi_{l, m}^{2} \epsilon^{m}$, $0 \leq l \leq \nu-1$, are equal to some formal series denoted $\hat{G}^{2}(\epsilon)=\sum_{m \geq 0} \varphi_{m}^{2} \epsilon^{m} \in \mathbb{E}[[\epsilon]]$. The Lemma 15 follows.

We consider now the bounded holomorphic functions

$$
a_{i}(\epsilon)=G_{i}(\epsilon)-\Psi_{i}^{1}(\epsilon)-\Psi_{i}^{2}(\epsilon)
$$

for all $0 \leq i \leq \nu-1$, all $\epsilon \in \mathcal{E}_{i}$. By definition, for $i \in I_{1}$ or $i \in I_{2}$, we have that

$$
a_{i+1}(\epsilon)-a_{i}(\epsilon)=G_{i+1}(\epsilon)-G_{i}(\epsilon)-\Delta_{i}^{1}(\epsilon)-\Delta_{i}^{2}(\epsilon)=G_{i+1}(\epsilon)-G_{i}(\epsilon)-\Delta_{i}(\epsilon)=0
$$

for all $\epsilon \in Z_{i}$. Therefore, each $a_{i}(\epsilon)$ is the restriction on $\mathcal{E}_{i}$ of a holomorphic function $a(\epsilon)$ on $D(0, r) \backslash\{0\}$. Since $a(\epsilon)$ is moreover bounded on $D(0, r) \backslash\{0\}$, the origin turns out to be a removable singularity for $a(\epsilon)$ which, as a consequence, defines a convergent power series on $D(0, r)$.

Finally, one can write the following decomposition

$$
G_{i}(\epsilon)=a(\epsilon)+\Psi_{i}^{1}(\epsilon)+\Psi_{i}^{2}(\epsilon)
$$

for all $\epsilon \in \mathcal{E}_{i}$, all $0 \leq i \leq \nu-1$. Moreover, $a(\epsilon)$ is a convergent power series, $\Psi_{i}^{1}(\epsilon)$ has the series $\hat{G}^{1}(\epsilon)=\sum_{m \geq 0} \varphi_{m}^{1} \epsilon^{m}$ as $1-$ Gevrey asymptotic expansion on $\mathcal{E}_{i}$ and $\Psi_{i}^{2}(\epsilon)$ carries the series $\hat{G}^{2}(\epsilon)=\sum_{m>0} \varphi_{m}^{2} \epsilon^{m}$ as $1^{+}$-Gevrey asymptotic expansion on $\mathcal{E}_{i}$, for all $0 \leq i \leq \nu-1$. The theorem (RS) follows. 


\subsection{Existence of formal series solutions in the complex parameter for the singularly perturbed problem}

We are now ready to state the main result of this paper.

Theorem 2 Let us assume that the conditions (115), (116), (117), (118) hold. Then, there exists a formal series

$$
\hat{X}(t, z, \epsilon)=\sum_{k \geq 0} H_{k}(t, z) \frac{\epsilon^{k}}{k !} \in \mathbb{E}[[\epsilon]]
$$

where the functions $H_{k}$ belong to the Banach space $\mathbb{E}=\mathcal{O}((\mathcal{T} \cap\{|t|>h\}) \times D(0, \delta / 2))$ of holomorphic and bounded functions on the set $(\mathcal{T} \cap\{|t|>h\}) \times D(0, \delta / 2)$ equipped with the supremum norm, for some $\delta, h>0$, which solves the singular equation

$$
\begin{aligned}
\epsilon \partial_{t} \partial_{z}^{S} \hat{X}(t, z, \epsilon) & +a \partial_{z}^{S} \hat{X}(t, z, \epsilon) \\
& =\sum_{\underline{k}=\left(s, k_{0}, k_{1}, k_{2}\right) \in \mathcal{A}_{1}} \frac{b_{\underline{k}}(z, \epsilon)}{t^{s+1}}\left(\partial_{t}^{k_{0}} \partial_{z}^{k_{1}} \hat{X}\right)\left(t+k_{2} \epsilon, z, \epsilon\right)+\sum_{l \in \mathcal{A}_{2}} c_{l}(z, \epsilon) \hat{X}^{l}(t, z, \epsilon)
\end{aligned}
$$

and owns the following properties. The formal series $\hat{X}$ can be decomposed into a sum of three terms

$$
\hat{X}(t, z, \epsilon)=a(t, z, \epsilon)+\hat{X}^{1}(t, z, \epsilon)+\hat{X}^{2}(t, z, \epsilon)
$$

where $a(t, z, \epsilon) \in \mathbb{E}\{\epsilon\}$ is a convergent series near $\epsilon=0$ and $\hat{X}^{1}(t, z, \epsilon), \hat{X}^{2}(t, z, \epsilon)$ belong to $\mathbb{E}[[\epsilon]]$. Moreover, for all $0 \leq i \leq \nu-1$, the $\mathbb{E}$-valued function $\epsilon \mapsto X_{U_{d_{i}}, \mathcal{E}_{i}}(t, z, \epsilon)$ constructed in Theorem 1 shares a similar decomposition

$$
X_{U_{d_{i}}, \mathcal{E}_{i}}(t, z, \epsilon)=a(t, z, \epsilon)+X_{U_{d_{i}}, \mathcal{E}_{i}}^{1}(t, z, \epsilon)+X_{U_{d_{i}}, \mathcal{E}_{i}}^{2}(t, z, \epsilon)
$$

where $\epsilon \mapsto X_{U_{d_{i}}, \mathcal{E}_{i}}^{1}(t, z, \epsilon)$ is a $\mathbb{E}$-valued function owning $\hat{X}^{1}(t, z, \epsilon)$ as 1 -Gevrey asymptotic expansion on $\mathcal{E}_{i}$ and where $\epsilon \mapsto X_{U_{d_{i}}, \mathcal{E}_{i}}^{2}(t, z, \epsilon)$ is a $\mathbb{E}$-valued function having $\hat{X}^{2}(t, z, \epsilon)$ as $1^{+}-$Gevrey asymptotic expansion on $\mathcal{E}_{i}$.

Proof Let us consider the tuple of functions $\left(X_{U_{d_{i}}, \mathcal{E}_{i}}(t, z, \epsilon)\right)_{0 \leq i \leq \nu-1}$ constructed in Theorem 1. For all $0 \leq i \leq \nu-1$, we define $G_{i}(\epsilon):=(t, z) \mapsto X_{U_{i},}, \mathcal{E}_{i}(t, z, \epsilon)$, which is, by construction, a holomorphic and bounded function from $\mathcal{E}_{i}$ into the Banach space $\mathbb{E}=\mathcal{O}((\mathcal{T} \cap\{|t|>h\}) \times$ $D(0, \delta / 2))$, where $\mathcal{T}, h, \delta$ are defined in Theorem 1 .

From the estimates (121), we get that the cocycle $\Delta_{i}(\epsilon)=G_{i+1}(\epsilon)-G_{i}(\epsilon)$ is exponentially flat on $Z_{i}=\mathcal{E}_{i+1} \cap \mathcal{E}_{i}$, for all $0 \leq i \leq \nu-1$. Moreover, if $d_{i}, d_{i+1} \in[-\pi,-\pi / 2) \cup(\pi / 2, \pi]$ and if $U_{d_{i}} \cap U_{d_{i+1}} \neq \emptyset$, then from (122) we deduce that $\Delta_{i}(\epsilon)=G_{i+1}(\epsilon)-G_{i}(\epsilon)$ is super-exponentially flat on $Z_{i}$. Hence, from the theorem (RS) stated above, there exist a formal series $\hat{G}(\epsilon) \in \mathbb{E}[[\epsilon]]$ which is the sum of a convergent series $a(\epsilon) \in \mathbb{E}\{\epsilon\}$ and two formal series $\hat{G}^{1}(\epsilon), \hat{G}^{2}(\epsilon) \in \mathbb{E}[[\epsilon]]$ such that $G_{i}(\epsilon)$ gets the corresponding decomposition

$$
G_{i}(\epsilon)=a(\epsilon)+G_{i}^{1}(\epsilon)+G_{i}^{2}(\epsilon)
$$

for all $\epsilon \in \mathcal{E}_{i}$, where $G_{i}^{1}(\epsilon)$ is holomorphic on $\mathcal{E}_{i}$ and has $\hat{G}^{1}(\epsilon)$ as 1-Gevrey asymptotic expansion on $\mathcal{E}_{i}$ and where $G_{i}^{2}(\epsilon)$ defines a holomorphic function on $\mathcal{E}_{i}$ owning $\hat{G}^{2}(\epsilon)$ as $1^{+}$-Gevrey asymptotic expansion on $\mathcal{E}_{i}$, for all $0 \leq i \leq \nu-1$. 
We put

$$
\hat{G}(\epsilon)=: \hat{X}(t, z, \epsilon)=\sum_{k \geq 0} H_{k}(t, z) \frac{\epsilon^{k}}{k !} .
$$

It remains to show that the formal series $\hat{X}(t, z, \epsilon)$ satisfies the equation (193). Let $0 \leq i \leq \nu-1$ such that $d_{i} \in(-\pi / 2, \pi / 2)$. From (194), $G_{i}(\epsilon)$ has in particular $\hat{G}(\epsilon)$ as 1 -Gevrey asymptotic expansion on $\mathcal{E}_{i}$ (since $1^{+}$-Gevrey asymptotic implies 1 -Gevrey asymptotic on sectors). As a result, we get

$$
\lim _{\epsilon \rightarrow 0, \epsilon \in \mathcal{E}_{i}} \sup _{(t, z) \in(\mathcal{T} \cap\{|t|>h\}) \times D(0, \delta / 2)}\left|\partial_{\epsilon}^{l} X_{U_{d_{i}}, \mathcal{E}_{i}}(t, z, \epsilon)-H_{l}(t, z)\right|=0
$$

for all $l \geq 0$. By the construction given in Theorem 1, the function $X_{U_{d_{i}}, \mathcal{E}_{i}}(t, z, \epsilon)$ satisfies the equation (100).

Now, we take the derivative of order $l$ with respect to $\epsilon$ of the left and the right hand side of the equation (100). From the Leibniz rule and the classical chain rule, we get that $\partial_{\epsilon}^{l} X_{U_{d_{i}}, \mathcal{E}_{i}}(t, z, \epsilon)$ satisfies the following equation

$$
\begin{gathered}
\epsilon \partial_{t} \partial_{z}^{S} \partial_{\epsilon}^{l} X_{U_{d_{i}}, \mathcal{E}_{i}}(t, z, \epsilon)+l \partial_{t} \partial_{z}^{S} \partial_{\epsilon}^{l-1} X_{U_{d_{i}}, \mathcal{E}_{i}}(t, z, \epsilon)+a \partial_{z}^{S} \partial_{\epsilon}^{l} X_{U_{d_{i}}, \mathcal{E}_{i}}(t, z, \epsilon) \\
=\sum_{\underline{k}=\left(s, k_{0}, k_{1}, k_{2}\right) \in \mathcal{A}_{1}} \frac{1}{t^{s+1}} \sum_{h_{1}+h_{2}=l} \frac{l !}{h_{1} ! h_{2} !} \partial_{\epsilon}^{h_{1}} b_{\underline{k}}(z, \epsilon) \\
\times\left(\sum_{h_{2,1}+h_{2,2}=h_{2}} \frac{h_{2} !}{h_{2,1} ! h_{2,2} !} k_{2}^{h_{2,1}}\left(\partial_{t}^{h_{2,1}+k_{0}} \partial_{z}^{k_{1}} \partial_{\epsilon}^{h_{2,2}} X_{U_{d_{i}}, \mathcal{E}_{i}}\right)\left(t+k_{2} \epsilon, z, \epsilon\right)\right) \\
\quad+\sum_{l_{1} \in \mathcal{A}_{2}} \sum_{h_{0}+\cdots+h_{l_{1}}=l} l ! \frac{\partial_{\epsilon}^{h_{0}} c_{l_{1}}(z, \epsilon)}{h_{0} !} \Pi_{j=1}^{l_{1}} \frac{\partial_{\epsilon}^{h_{j}} X_{U_{d_{i}}, \mathcal{E}_{i}}(t, z, \epsilon)}{h_{j} !}
\end{gathered}
$$

for all $l \geq 1$, all $(t, z, \epsilon) \in(\mathcal{T} \cap\{|t|>h\}) \times D(0, \delta / 2) \times \mathcal{E}_{i}$. Letting $\epsilon$ tends to zero in (196) and using (195) yields the recursion

$$
\begin{aligned}
& \partial_{t} \partial_{z}^{S}\left(\frac{H_{l-1}(t, z)}{(l-1) !}\right)+a \partial_{z}^{S}\left(\frac{H_{l}(t, z)}{l !}\right)= \sum_{\underline{k}=\left(s, k_{0}, k_{1}, k_{2}\right) \in \mathcal{A}_{1}} \frac{1}{t^{s+1}} \sum_{h_{1}+h_{2}=l} \frac{\left(\partial_{\epsilon}^{h_{1}} b_{\underline{k}}\right)(z, 0)}{h_{1} !} \\
& \times\left(\sum_{h_{2,1}+h_{2,2}=h_{2}} \frac{1}{h_{2,1} ! h_{2,2} !} k_{2}^{h_{2,1}}\left(\partial_{t}^{h_{2,1}+k_{0}} \partial_{z}^{k_{1}} H_{h_{2,2}}\right)(t, z)\right) \\
&+\sum_{l_{1} \in \mathcal{A}_{2} \sum_{h_{0}+\cdots+h_{l_{1}}=l} \frac{\left(\partial_{\epsilon}^{h_{0}} c_{l_{1}}\right)(z, 0)}{h_{0} !} \Pi_{j=1}^{l_{1}} \frac{H_{h_{j}}(t, z)}{h_{j} !}}
\end{aligned}
$$

for all $l \geq 1$, all $(t, z) \in(\mathcal{T} \cap\{|t|>h\}) \times D(0, \delta / 2)$.

Since $b_{\underline{k}}(z, \epsilon)$ and $c_{l_{1}}(z, \epsilon)$ are analytic with respect to $\epsilon$ at 0 , we have that

$$
b_{\underline{k}}(z, \epsilon)=\sum_{h \geq 0} \frac{\left(\partial_{\epsilon}^{h} b_{\underline{k}}\right)(z, 0)}{h !} \epsilon^{h} \quad, \quad c_{l_{1}}(z, \epsilon)=\sum_{h \geq 0} \frac{\left(\partial_{\epsilon}^{h} c_{l_{1}}\right)(z, 0)}{h !} \epsilon^{h}
$$

On the other hand, by taking the Taylor expansion of the function $\left(\partial_{t}^{k_{0}} \partial_{z}^{k_{1}} H_{k}\right)(\xi, z)$ at the point $\xi=t$, we get that

$$
\left(\partial_{t}^{k_{0}} \partial_{z}^{k_{1}} H_{k}\right)\left(t+k_{2} \epsilon, z\right)=\sum_{h \geq 0} \frac{\left(\partial_{t}^{k_{0}+h} \partial_{z}^{k_{1}} H_{k}\right)(t, z)}{h !} k_{2}^{h} \epsilon^{h}
$$


for all $\epsilon$ near the origin, for all $k \geq 0$. Therefore, one can write

$$
\begin{aligned}
&\left(\partial_{t}^{k_{0}} \partial_{z}^{k_{1}} \hat{X}\right)\left(t+k_{2} \epsilon, z, \epsilon\right)=\sum_{k \geq 0} \sum_{h \geq 0} \frac{\left(\partial_{t}^{k_{0}+h} \partial_{z}^{k_{1}} H_{k}\right)(t, z)}{h !} k_{2}^{h} \frac{\epsilon^{h+k}}{k !} \\
&=\sum_{l \geq 0}\left(\sum_{l_{1}+l_{2}=l} \frac{k_{2}^{l_{1}}\left(\partial_{t}^{k_{0}+l_{1}} \partial_{z}^{k_{1}} H_{l_{2}}\right)(t, z)}{l_{1} ! l_{2} !}\right) \epsilon^{l}
\end{aligned}
$$

as a formal power series in $\epsilon$. Finally, using the recursion (197) together with the expansions (198), (200), one checks that the formal series $\hat{X}(t, z, \epsilon)$ solves the equation (193).

\section{References}

[1] Z. Artstein, M. Slemrod, On singularly perturbed retarded functional-differential equations. J. Differential Equations 171 (2001), no. 1, 88-109.

[2] W. Balser, From divergent power series to analytic functions. Theory and application of multisummable power series. Lecture Notes in Mathematics, 1582. Springer-Verlag, Berlin, 1994.

[3] W. Balser, Formal power series and linear systems of meromorphic ordinary differential equations, Springer-Verlag, New-York, 2000.

[4] B. Braaksma, Multisummability of formal power series solutions of nonlinear meromorphic differential equations. Ann. Inst. Fourier (Grenoble) 42 (1992), no. 3, 517-540.

[5] B. Braaksma, B. Faber, Multisummability for some classes of difference equations. Ann. Inst. Fourier (Grenoble) 46 (1996), no. 1, 183-217.

[6] B. Braaksma, B. Faber, G. Immink, Summation of formal solutions of a class of linear difference equations. Pacific J. Math. 195 (2000), no. 1, 35-65.

[7] M. Canalis-Durand, J. Mozo-Fernandez, R. Schäfke, Monomial summability and doubly singular differential equations. J. Differential Equations 233 (2007), no. 2, 485-511.

[8] M. Canalis-Durand, J-P. Ramis, R. Schäfke, Y. Sibuya, Gevrey solutions of singularly perturbed differential equations. J. Reine Angew. Math. 518 (2000), 95-129.

[9] O. Costin, Asymptotics and Borel summability, Chapman Hall/CRC Monographs and Surveys in Pure and Applied Mathematics, 141. CRC Press, Boca Raton, FL, 2009.

[10] O. Costin, S. Tanveer, Existence and uniqueness for a class of nonlinear higher-order partial differential equations in the complex plane. Comm. Pure Appl. Math. 53 (2000), no. 9, 10921117.

[11] J. Dieudonné, Calcul infinitésimal. (French) Hermann, Paris 1968479 pp.

[12] A. El-Rabih, R. Schäfke, Overstable analytic solutions for non-linear systems of difference equations with small step size containing an additional parameter. J. Difference Equ. Appl. 11 (2005), no. 3, 183-213. 
[13] A. Fowler, Asymptotic methods for delay equations. J. Engrg. Math. 53 (2005), no. 3-4, 271-290.

[14] A. Fruchard, R. Schäfke, Bifurcation delay and difference equations. Nonlinearity 16 (2003), no. $6,2199-2220$.

[15] A. Fruchard, R. Schäfke, Analytic solutions of difference equations with small step size. In memory of W. A. Harris, J. Differ. Equations Appl. 7 (2001), no. 5, 651-684.

[16] V. Glizer, Blockwise estimate of the fundamental matrix of linear singularly perturbed differential systems with small delay and its application to uniform asymptotic solution. J. Math. Anal. Appl. 278 (2003), no. 2, 409-433.

[17] P. Hsieh, Y. Sibuya, Basic theory of ordinary differential equations. Universitext. SpringerVerlag, New York, 1999.

[18] G. Immink, Accelero-summation of the formal solutions of nonlinear difference equations. Ann. Inst. Fourier (Grenoble) 61 (2011), no. 1, 1-51.

[19] G. Immink, Exact asymptotics of nonlinear difference equations with levels 1 and $1+$. Ann. Fac. Sci. Toulouse Math. (6) 17 (2008), no. 2, 309-356.

[20] G. Immink, Asymptotic expansions with error bounds for solutions of difference equations of "level 1+". Équations différentielles dans le champ complexe, Vol. I (Strasbourg, 1985), 35-60, Publ. Inst. Rech. Math. Av., Univ. Louis Pasteur, Strasbourg, 1988.

[21] A. Lastra, S. Malek, On q-Gevrey asymptotics for singularly perturbed q-differencedifferential problems with an irregular singularity, Abstract and Applied Analysis, vol. 2012, Article ID 860716.

[22] A. Lastra, S. Malek, J. Sanz, Continuous right inverses for the asymptotic Borel map in ultraholomorphic classes via a Laplace-type transform, Journal of Mathematical Analysis and Applications, vol 396, no. 2 (2012).

[23] S. Malek, On the parametric Stokes phenomenon for solutions of singularly perturbed linear partial differential equations, Abstract and Applied Analysis, vol. 2012, Article ID 930385.

[24] S. Malek, On the summability of formal solutions for doubly singular nonlinear partial differential equations J. Dynam. Control. Syst. 18 (2012), no. 1.

[25] S. Malek, Singularly perturbed q-difference-differential equations with irregular singularity, J. Dynam. Control. Syst. 17 (2011), no. 2.

[26] B. Malgrange and J.-P. Ramis, Fonctions multisommables, Ann. Inst. Fourier, Grenoble, 42-1 (1992), 353-368.

[27] J. Melenk, C. Schwab, Analytic regularity for a singularly perturbed problem. SIAM J. Math. Anal. 30 (1999), no. 2, 379-400.

[28] Jia-qi Mo, Zhao-hui Wen, Singularly perturbed reaction diffusion equations with time delay. Appl. Math. Mech. (English Ed.) 31 (2010), no. 6, 769-774.

[29] F. Olver, Asymptotics and special functions. Computer Science and Applied Mathematics. Academic Press [A subsidiary of Harcourt Brace Jovanovich, Publishers], New YorkLondon, 1974. xvi+572 pp. 
[30] J.-P. Ramis, Y. Sibuya, A new proof of multisummability of formal solutions of nonlinear meromorphic differential equations. Ann. Inst. Fourier (Grenoble) 44 (1994), no. 3, 811-848.

[31] H. Tian, Asymptotic expansion for the solution of singularly perturbed delay differential equations. J. Math. Anal. Appl. 281 (2003), no. 2, 678-696.

[32] Z. Xiang, Singular perturbation of initial-boundary value problems of reaction diffusion equation with delay. Appl. Math. Mech. (English Ed.) 15 (1994), no. 3, 267-273. 